\title{
Quilted Floer cohomology
}

\author{
KATRIN WEHRHEIM \\ CHRIS T WOODWARD
}

\begin{abstract}
We generalize Lagrangian Floer cohomology to sequences of Lagrangian correspondences. For sequences related by the geometric composition of Lagrangian correspondences we establish an isomorphism of the Floer cohomologies. This provides the foundation for the construction of a symplectic 2-category as well as for the definition of topological invariants via decomposition and representation in the symplectic category. Here we give some first direct symplectic applications: Calculations of Floer cohomology, displaceability of Lagrangian correspondences and transfer of displaceability under geometric composition.
\end{abstract}

53D40; 57R56

\section{Introduction}

Lagrangian Floer cohomology associates to a pair of compact Lagrangian manifolds a chain complex whose differential counts pseudoholomorphic strips with boundary values in the given Lagrangians. In this paper we generalize Floer cohomology to include compact Lagrangian correspondences. Recall that if $\left(M_{0}, \omega_{0}\right)$ and $\left(M_{1}, \omega_{1}\right)$ are symplectic manifolds, then a Lagrangian correspondence $L_{01}$ from $M_{0}$ to $M_{1}$ is a Lagrangian submanifold $L_{01} \subset M_{0}^{-} \times M_{1}$, where $M_{0}^{-}:=\left(M_{0},-\omega_{0}\right)$. These were introduced by Weinstein [45] in an attempt to create a symplectic category with morphisms between not necessarily symplectomorphic manifolds. So we also denote a Lagrangian correspondence by

$$
M_{0} \stackrel{L_{01}}{\longrightarrow} M_{1} \text {. }
$$

With this notation we can view a pair of Lagrangian submanifolds $L, L^{\prime} \subset M$ as sequence of Lagrangian correspondences

$$
\mathrm{pt} \stackrel{L}{\longrightarrow} M \stackrel{L^{\prime}}{\longrightarrow} \mathrm{pt}
$$

from the point via $M$ back to the point. This is a special case of a cyclic sequence of Lagrangian correspondences

$$
M_{0} \stackrel{L_{01}}{\longrightarrow} M_{1} \stackrel{L_{12}}{\longrightarrow} M_{2} \cdots M_{r} \stackrel{L_{r(r+1)}}{\longrightarrow} M_{r+1}=M_{0}
$$


for which we will define a quilted Floer cohomology

$$
H F\left(L_{01}, L_{12}, \ldots, L_{r(r+1)}\right) .
$$

The quilted Floer complex is generated by generalized intersection points. The quilted differential counts tuples of pseudoholomorphic strips $\left(u_{j}: \mathbb{R} \times[0,1] \rightarrow M_{j}\right)_{j=0, \ldots, r}$ whose boundaries match up via the Lagrangian correspondences $\left(u_{j}(s, 1), u_{j+1}(s, 0)\right) \in$ $L_{j(j+1)}$ for $j=0, \ldots, r$. These tuples are examples of pseudoholomorphic quilts with the strips thought of as patches and the boundary matching conditions thought of as seams. We develop the theory of quilts in higher generality in [41]. In this paper, we next investigate the effect of geometric composition on Floer cohomology. The geometric composition of two Lagrangian correspondences $L_{01} \subset M_{0}^{-} \times M_{1}$, $L_{12} \subset M_{1}^{-} \times M_{2}$ is

$$
L_{01} \circ L_{12}:=\left\{\left(x_{0}, x_{2}\right) \in M_{0} \times M_{2} \mid \exists x_{1}:\left(x_{0}, x_{1}\right) \in L_{01},\left(x_{1}, x_{2}\right) \in L_{12}\right\} .
$$

In general, this will be a singular subset of $M_{0}^{-} \times M_{2}$. However, if we assume transversality of the intersection

$$
L_{01} \times_{M_{1}} L_{12}:=\left(L_{01} \times L_{12}\right) \cap\left(M_{0}^{-} \times \Delta_{M_{1}} \times M_{2}\right),
$$

then the restriction of the projection $\pi_{02}: M_{0}^{-} \times M_{1} \times M_{1}^{-} \times M_{2} \rightarrow M_{0}^{-} \times M_{2}$ to $L_{01} \times_{M_{1}} L_{12}$ is automatically a Lagrangian immersion. We will study the class of embedded geometric compositions, for which in addition $\pi_{02}$ is injective, and hence $L_{01} \circ L_{12}$ is a smooth Lagrangian correspondence. If the composition $L_{(\ell-1) \ell} \circ L_{\ell(\ell+1)}$ is embedded, then we obtain under suitable monotonicity assumptions a canonical isomorphism

$$
H F\left(\ldots, L_{(\ell-1) \ell}, L_{\ell(\ell+1)}, \ldots\right) \cong H F\left(\ldots, L_{(\ell-1) \ell} \circ L_{\ell(\ell+1)}, \ldots\right) .
$$

For the precise monotonicity and admissibility conditions see Section 5.4. The proof proceeds in two steps. First, we allow for varying widths $\left(\delta_{j}>0\right)_{j=0, \ldots, r}$ of the pseudoholomorphic strips $\left(u_{j}: \mathbb{R} \times\left[0, \delta_{j}\right] \rightarrow M_{j}\right)_{j=0, \ldots, r}$ defining the differential. Section 5.3 of this paper shows that Floer cohomology is independent of the choice of widths. (These domains are not conformally equivalent due to the identification between boundary components that is implicit in the seam conditions.) The second (hard analytic) part is to prove that with the width $\delta_{\ell}>0$ sufficiently close to zero, the $(r+1)-$ tuples of holomorphic strips with seam conditions in $\left(\ldots, L_{(\ell-1) \ell}, L_{\ell(\ell+1)}, \ldots\right)$ are in one-to-one correspondence with the $(r+1)$-tuples of holomorphic strips with seam conditions in $\left(\ldots, L_{(\ell-1) \ell} \circ L_{\ell(\ell+1)}, \ldots\right)$. This analysis is completely analogous to our work [38], where we establish the bijection for the Floer trajectories of the special 
cyclic sequence

$$
\mathrm{pt} \stackrel{L_{0}}{\longrightarrow} M_{0} \stackrel{L_{01}}{\longrightarrow} M_{1} \stackrel{L_{12}}{\longrightarrow} M_{2} \stackrel{L_{2}}{\longrightarrow} \mathrm{pt}
$$

when $\delta_{1} \rightarrow 0$. The monotonicity assumptions are crucial for this part since the exclusion of a novel "figure eight bubble" in [38] hinges on a strict energy-index proportionality.

In Section 6 we provide a number of new tools for the calculation of Floer cohomology (and hence detection of nondisplaceability), arising as direct consequences of (3) or from a conjectural generalization of Perutz' long exact Gysin sequence [24]. These are meant to exemplify the wide applicability and reach of the basic isomorphism (3). We believe that it should have much more dramatic consequences once systematically employed. As first specific example of direct consequences of (3) we confirm the calculation $H F\left(T_{\mathrm{Cl}}^{n}, T_{\mathrm{Cl}}^{n}\right) \cong H_{*}\left(T^{n}\right)$ of Cho [4] for the Clifford torus in $\mathbb{C P}^{n}$, and we calculate some further Floer cohomologies in $\mathbb{C P}^{n}$ using reduction at pairs of transverse level sets. Next, we prove Hamiltonian nondisplaceability of the Lagrangian 3-sphere $\Sigma \subset\left(\mathbb{C} \mathbb{P}^{1}\right)^{-} \times \mathbb{C P}^{2}$ arising from reduction at the level set of an $S^{1}$-action on $\mathbb{C P}^{2}$ containing $T_{\mathrm{Cl}}$. The latter will be deduced from the nontriviality of $H F\left(T_{\mathrm{Cl}}^{2}, T_{\mathrm{Cl}}^{2}\right)$ together with our isomorphism

$$
H F\left(S^{1} \times T_{\mathrm{Cl}}, \Sigma\right) \cong H F\left(T_{\mathrm{Cl}}, T_{\mathrm{Cl}}\right),
$$

and the fact that $H F\left(L^{\prime}, L\right) \neq 0 \Rightarrow H F(L, L) \neq 0$ (since $H F(L, L)$ contains an element that acts as the identity on $\left.H F\left(L^{\prime}, L\right)\right)$. Finally, we generalize this nondisplaceability result to the Lagrangian embedding $\Sigma \subset\left(\mathbb{C P}^{k-1}\right)^{-} \times \mathbb{C} \mathbb{P}^{n}$ of $\left(S^{1}\right)^{n-k} \times S^{2 k-1}$, which arises from the monotone level set of an $S^{n-k+1}$-action on $\mathbb{C} \mathbb{P}^{n}$ for $2 \leq k \leq n$.

Another consequence of our results is a general prescription for defining topological invariants by decomposing into simple pieces. For example, let $Y$ be a compact manifold and $f: Y \rightarrow \mathbb{R}$ a Morse function, which induces a decomposition $Y=Y_{01} \cup \cdots \cup Y_{(k-1) k}$ into simple cobordisms by cutting along noncritical level sets $X_{1}, \ldots, X_{k-1}$. First one associates to each $X_{j}$ a monotone symplectic manifold $M\left(X_{j}\right)$, and to each $Y_{(j-1) j}$ with $\partial Y_{(j-1) j}=X_{j-1}^{-} \sqcup X_{j}$ a smooth monotone Lagrangian correspondence $L\left(Y_{(j-1) j}\right) \subset M\left(X_{j-1}\right)^{-} \times M\left(X_{j}\right)$ (taking $M\left(X_{0}\right)$ and $M\left(X_{k}\right)$ to be points.) Second, one checks that the basic moves described by Cerf theory (cancellation or change of order of critical points) change the sequence of Lagrangian correspondences by replacing adjacent correspondences with an embedded composition, or vice-versa. In other words, the equivalence class of sequences of Lagrangian correspondences by embedded compositions $\left[L\left(Y_{01}\right), \ldots, L\left(Y_{(k-1) k}\right)\right]$ does not depend on the choice of the Morse function $f$. Then the results of this paper provide a group-valued invariant of $Y$, by taking the Floer homology of the sequence of Lagrangian correspondences. For example, in [42; 43] we investigate the theory 
which uses as symplectic manifolds the moduli spaces of flat bundles with compact structure group on three-dimensional cobordisms containing tangles. Conjecturally this provides a symplectic construction of Donaldson type gauge theoretic invariants: $\mathrm{SO}(3)$-instanton Floer homology, its higher rank version (though not strictly defined in the gauge theoretic setting), and the SU(n)-tangle invariants defined by KronheimerMrowka [14] from singular instantons. The same setup is used to give alternative constructions of Heegaard Floer homology [16] and Seidel-Smith invariants [26].

Even more generally, in our paper [39] the quilted Floer cohomology groups provide the 2-morphism spaces in our construction of a symplectic 2-category which contains all Lagrangian correspondences as morphisms, and where the composition is equivalent to geometric composition, if the latter is embedded. This setup in turn is used by Abouzaid-Smith [1] to prove mirror symmetry for the 4-torus and deduce various further symplectic consequences.

Notation and organization We will frequently refer to the assumptions (M1)-(M2), (L1)-(L3) and (G1)-(G2) that can be found at the beginning of Section 4.1.

Section 2 is a detailed introduction to Lagrangian correspondences, geometric composition and sequences of correspondences, which also provides the basic framework for the sequels $[39 ; 41]$ to this paper. In Section 3 we generalize gradings to Lagrangian correspondences and establish their behaviour under geometric composition, so that the isomorphism (3) becomes an isomorphism of graded groups. Section 4 provides a review of monotonicity and Floer cohomology and gives a first definition of the Floer cohomology (1) by building a pair of Lagrangians in the product $M_{0} \times M_{1} \times \cdots M_{k-1}$. The latter is however unsatisfactory since it does not provide an approach to the isomorphism (3). Section 5 gives the general definition of quilted Floer cohomology (1) and finalizes the proof of the isomorphism (3). Finally, Section 6 gives a number of direct symplectic applications of the isomorphism (3).

Acknowledgements We thank Paul Seidel and Ivan Smith for encouragement and helpful discussions, and we are very grateful to the meticulous referee for help with cleaning up the details.

\section{Lagrangian correspondences}

Let $M$ be a smooth manifold. A symplectic form on $M$ is a closed, nondegenerate two-form $\omega$. A symplectic manifold is a smooth manifold equipped with a symplectic form. If $\left(M_{1}, \omega_{1}\right)$ and $\left(M_{2}, \omega_{2}\right)$ are symplectic manifolds, then a diffeomorphism $\varphi: M_{1} \rightarrow M_{2}$ is a symplectomorphism if $\varphi^{*} \omega_{2}=\omega_{1}$. Let Symp denote the category 
whose objects are symplectic manifolds and whose morphisms are symplectomorphisms. We have the following natural operations on Symp.

(a) (Duals) If $M=(M, \omega)$ is a symplectic manifold, then $M^{-}=(M,-\omega)$ is a symplectic manifold, called the dual of $M$.

(b) (Sums) If $M_{j}=\left(M_{j}, \omega_{j}\right), j=1,2$ are symplectic manifolds, then the disjoint union $M_{1} \cup M_{2}$ equipped with the symplectic structure $\omega_{1}$ on $M_{1}$ and $\omega_{2}$ on $M_{2}$, is a symplectic manifold. The empty set $\varnothing$ is a unit for the disjoint union.

(c) (Products) If $M_{j}=\left(M_{j}, \omega_{j}\right), j=1,2$ are symplectic manifolds, the Cartesian product $\left(M_{1} \times M_{2}, \pi_{1}^{*} \omega_{1}+\pi_{2}^{*} \omega_{2}\right)$ is a symplectic manifold. (Here $\pi_{j}: M_{1} \times M_{2} \rightarrow$ $M_{j}$ denotes the projections.) The symplectic manifold pt, consisting of a single point, is a unit for the Cartesian product.

Clearly the notion of symplectomorphism is very restrictive; in particular, the symplectic manifolds must be of the same dimension. A more flexible notion of morphism is that of Lagrangian correspondence, defined as follows [44; 45; 12]. Let $M=(M, \omega)$ be a symplectic manifold. A submanifold $L \subset M$ is isotropic, resp. coisotropic, resp. Lagrangian if the $\omega$-orthogonal complement $T L^{\omega}$ satisfies $T L^{\omega} \supseteq T L$ resp. $T L^{\omega} \subseteq T L$ resp. $T L^{\omega}=T L$.

Definition 2.0.1 Let $M_{1}, M_{2}$ be symplectic manifolds. A Lagrangian correspondence from $M_{1}$ to $M_{2}$ is a Lagrangian submanifold $L_{12} \subset M_{1}^{-} \times M_{2}$.

Example 2.0.2 The following are examples of Lagrangian correspondences:

(a) (Trivial correspondence) The one and only Lagrangian correspondence between $M_{1}=\varnothing$ and any other $M_{2}$ is $L_{12}=\varnothing$.

(b) (Lagrangians) Any Lagrangian submanifold $L \subset M$ can be viewed both as correspondence $L \subset \mathrm{pt}^{-} \times M$ from the point to $M$ and as correspondence $L \subset M^{-} \times \mathrm{pt}$ from $M$ to the point.

(c) (Graphs) If $\varphi_{12}: M_{1} \rightarrow M_{2}$ is a symplectomorphism then its graph

$$
\operatorname{graph}\left(\varphi_{12}\right)=\left\{\left(m_{1}, \varphi_{12}\left(m_{1}\right)\right) \mid m_{1} \in M_{1}\right\} \subset M_{1}^{-} \times M_{2}
$$

is a Lagrangian correspondence.

(d) (Fibered coisotropics) Suppose that $\iota: C \rightarrow M$ is a coisotropic submanifold. Then the null distribution $T C^{\omega}$ is integrable; see eg McDuff and Salamon [18, Lemma 5.30]. Suppose that $T C^{\omega}$ is in fact fibrating, that is, there exists a symplectic manifold $\left(B, \omega_{B}\right)$ and a fibration $\pi: C \rightarrow B$ such that $\iota^{*} \omega$ is the pullback $\pi^{*} \omega_{B}$. Then

$$
(\iota \times \pi): C \rightarrow M^{-} \times B
$$


maps $C$ to a Lagrangian correspondence.

(e) (Level sets of moment maps) Let $G$ be a Lie group with Lie algebra $\mathfrak{g}$. Suppose that $G$ acts on $M$ by Hamiltonian symplectomorphisms generated by a moment map $\mu: M \rightarrow \mathfrak{g}^{*}$. (That is $\mu$ is equivariant and the generating vector fields $\mathfrak{g} \rightarrow \operatorname{Vect}(M), \xi \mapsto \xi_{M}$ satisfy $\iota\left(\xi_{M}\right) \omega=-d(\mu, \xi)$.) If $G$ acts freely on $\mu^{-1}(0)$, then $\mu^{-1}(0)$ is a smooth coisotropic fibered over the symplectic quotient $M / / G=$ $\mu^{-1}(0) / G$, which is a symplectic manifold. Hence we have a Lagrangian correspondence

$$
(\iota \times \pi): \mu^{-1}(0) \rightarrow M^{-} \times(M / / G) .
$$

The symplectic two-form $\omega_{M / G}$ on $M / / G$ is the unique form on $M / / G$ satisfying $\pi^{*} \omega_{M / G}=\iota^{*} \omega$.

Definition 2.0.3 Let $M_{0}, M_{1}, M_{2}$ be symplectic manifolds and $L_{01} \subset M_{0}^{-} \times M_{1}$, $L_{12} \subset M_{1}^{-} \times M_{2}$ Lagrangian correspondences.

(a) The dual Lagrangian correspondence of $L_{01}$ is

$$
\left(L_{01}\right)^{t}:=\left\{\left(m_{1}, m_{0}\right) \mid\left(m_{0}, m_{1}\right) \in L_{01}\right\} \subset M_{1}^{-} \times M_{0} .
$$

(b) The geometric composition of $L_{01}$ and $L_{12}$ is

$L_{01} \circ L_{12}:=\left\{\left(m_{0}, m_{2}\right) \in M_{0}^{-} \times M_{2} \mid \exists m_{1} \in M_{1}: \begin{array}{l}\left(m_{0}, m_{1}\right) \in L_{01} \\ \left(m_{1}, m_{2}\right) \in L_{12}\end{array}\right\} \subset M_{0}^{-} \times M_{2}$.

Geometric composition and duals of Lagrangian correspondences satisfy the following:

(a) (Graphs) If $\varphi_{01}: M_{0} \rightarrow M_{1}$ and $\varphi_{12}: M_{1} \rightarrow M_{2}$ are symplectomorphisms, then

$$
\begin{aligned}
& \operatorname{graph}\left(\varphi_{01}\right) \circ \operatorname{graph}\left(\varphi_{12}\right)=\operatorname{graph}\left(\varphi_{12} \circ \varphi_{01}\right), \\
& \operatorname{graph}\left(\varphi_{01}\right)^{t}=\operatorname{graph}\left(\varphi_{01}^{-1}\right) .
\end{aligned}
$$

(b) (Zero) Composition with $\varnothing$ always yields $\varnothing$, that is for any Lagrangian correspondence $L_{01} \subset M_{0}^{-} \times M_{1}$ we have

$$
\varnothing \circ L_{01}=\varnothing, \quad L_{01} \circ \varnothing=\varnothing .
$$

(c) (Identity) The diagonal is an identity. That is, if $L_{01} \subset M_{0}^{-} \times M_{1}$ is a Lagrangian correspondence and $\Delta_{j} \subset M_{j}^{-} \times M_{j}$ are the diagonals, then

$$
L_{01}=\Delta_{0} \circ L_{01}=L_{01} \circ \Delta_{1} \text {. }
$$


(d) (Associativity) If $L_{01} \subset M_{0}^{-} \times M_{1}, L_{12} \subset M_{1}^{-} \times M_{2}, L_{23} \subset M_{2}^{-} \times M_{3}$ are Lagrangian correspondences, then

$$
\begin{gathered}
\left(L_{01} \circ L_{12}\right) \circ L_{23}=L_{01} \circ\left(L_{12} \circ L_{23}\right), \\
\left(L_{01} \circ L_{12}\right)^{t}=\left(L_{12}\right)^{t} \circ\left(L_{01}\right)^{t} .
\end{gathered}
$$

The geometric composition can equivalently be defined as

$$
L_{01} \circ L_{12}=\pi_{02}\left(L_{01} \times_{M_{1}} L_{12}\right),
$$

the image under the projection $\pi_{02}: M_{0}^{-} \times M_{1} \times M_{1}^{-} \times M_{2} \rightarrow M_{0}^{-} \times M_{2}$ of

$$
L_{12} \times M_{1} L_{01}:=\left(L_{01} \times L_{12}\right) \cap\left(M_{0}^{-} \times \Delta_{1} \times M_{2}\right) .
$$

Here $\Delta_{1} \subset M_{1}^{-} \times M_{1}$ denotes the diagonal. $L_{01} \circ L_{12} \subset M_{0}^{-} \times M_{2}$ is an immersed Lagrangian submanifold if $L_{01} \times L_{12}$ intersects $M_{0}^{-} \times \Delta_{1} \times M_{2}$ transversally. In general, the geometric composition of smooth Lagrangian submanifolds may not even be immersed. We will be working with the following class of compositions, for which the resulting Lagrangian correspondence is in fact a smooth submanifold, as will be seen in Lemma 2.0.5 below.

Definition 2.0.4 We say that the composition $L_{01} \circ L_{12}$ is embedded if $L_{12} \times_{M_{1}} L_{01}$ is cut out transversally (ie $\left.\left(L_{01} \times L_{12}\right) \pitchfork\left(M_{0}^{-} \times \Delta_{1} \times M_{2}\right)\right)$ and the projection $\pi_{02}: L_{12} \times_{M_{1}} L_{01} \rightarrow L_{01} \circ L_{12} \subset M_{0}^{-} \times M_{2}$ is an embedding. (For compact Lagrangians it suffices to assume that $\pi_{02}$ is injective, by Lemma 2.0.5 below.)

By some authors (eg Guillemin and Sternberg [12]) geometric composition of Lagrangian correspondences is more generally defined under clean intersection hypotheses. This extension is not needed in the present paper, because the quilted Floer cohomology is invariant under Hamiltonian isotopy, and after such an isotopy transversality may always be achieved. However, transverse intersection only yields an immersed ${ }^{1}$ Lagrangian correspondence, as the following Lemma shows.

Lemma 2.0.5 Let $L_{01} \subset M_{0}^{-} \times M_{1}, L_{12} \subset M_{1}^{-} \times M_{2}$ be Lagrangian correspondences such that the intersection $\left(L_{01} \times L_{12}\right) \pitchfork\left(M_{0}^{-} \times \Delta_{1} \times M_{2}\right)$ is transverse. Then the projection $\pi_{02}: L_{12} \times_{M_{1}} L_{01} \rightarrow L_{01} \circ L_{12} \subset M_{0}^{-} \times M_{2}$ is an immersion.

In particular, if the Lagrangians are compact, the intersection is transverse, and the projection is injective, then the composition $L_{01} \circ L_{12}=: L_{02}$ is embedded.

\footnotetext{
${ }^{1}$ One can not necessarily remove all self-intersections of the immersed composition by Hamiltonian isotopy on one correspondence. A basic example is the composition of transverse Lagrangian submanifolds $L, L^{\prime} \subset M$. Identifying $M \cong M \times\{\mathrm{pt}\} \cong\{\mathrm{pt}\} \times M$ the projection $L \times_{M} L^{\prime} \rightarrow L \circ L^{\prime} \subset\{\mathrm{pt}\} \times\{\mathrm{pt}\}$ maps the (finite) intersection $L \pitchfork L^{\prime}$ to a point.
} 
Proof The proof essentially is a special case of the fact that the geometric composition of linear Lagrangian correspondences is always well defined (ie yields another linear Lagrangian correspondence); see eg Guillemin and Sternberg [12, Section 4.1].

Fix a point $\underline{x}=\left(x_{0}, x_{1}, x_{1}, x_{2}\right) \in L_{01} \times_{M_{1}} L_{12}$ then we need to check that $\operatorname{ker} \mathrm{d}_{\underline{x}} \pi_{02}=$ $\{0\}$ for the projection restricted to $L_{12} \times_{M_{1}} L_{01}$. In fact, we will show that

(4) $\operatorname{kerd}_{\underline{x}} \pi_{02} \cong \frac{T_{\underline{x}}\left(M_{0} \times M_{1} \times M_{1} \times M_{2}\right)}{\left(T_{\left(x_{0}, x_{1}\right)} L_{01} \times T_{\left(x_{1}, x_{2}\right)} L_{12}\right)+\left(T_{x_{0}} M_{0} \times T_{\left(x_{1}, x_{1}\right)} \Delta_{1} \times T_{x_{2}} M_{2}\right)}$,

which is zero by transversality. To simplify notation we abbreviate $\Lambda_{01}:=T_{\left(x_{0}, x_{1}\right)} L_{01}$, $\Lambda_{12}:=T_{\left(x_{1}, x_{2}\right)} L_{12}$, and $V_{i}:=T_{x_{i}} M_{i}$. Now (4) follows as in [12, Section 4.1]. For completeness we recall the precise argument: We identify

$$
\begin{aligned}
\operatorname{ker} d_{\underline{x}} \pi_{02} & =\left(\Lambda_{01} \times_{V_{1}} \Lambda_{12}\right) \cap\left(\{0\} \times V_{1} \times V_{1} \times\{0\}\right) \\
& \cong\left\{v_{1} \in V_{1} \mid\left(0, v_{1}\right) \in \Lambda_{01},\left(v_{1}, 0\right) \in \Lambda_{12}\right\}=\operatorname{ker} \Lambda_{01}^{t} \cap \operatorname{ker} \Lambda_{12},
\end{aligned}
$$

where $\operatorname{ker} \Lambda_{12}:=\left\{v_{1} \in V_{1} \mid\left(v_{1}, 0\right) \in \Lambda_{12}\right\} \subset V_{1}$ and similarly $\operatorname{ker} \Lambda_{01}^{t} \subset V_{1}$. On the other hand, we use the symplectic complements with respect to the form $\omega_{0112}:=$ $\left(-\omega_{0}\right) \oplus \omega_{1} \oplus\left(-\omega_{1}\right) \oplus \omega_{2}$ on $V_{0} \times V_{1} \times V_{1} \times V_{2}$ to identify

$\left(V_{0} \times V_{1} \times V_{1} \times V_{2}\right) /\left(\Lambda_{01} \times \Lambda_{12}\right)+\left(V_{0} \times \Delta_{V_{1}} \times V_{2}\right)$

$$
\begin{aligned}
& \cong\left(\Lambda_{01} \times \Lambda_{12}\right)^{\omega_{0112}} \cap\left(V_{0} \times \Delta_{V_{1}} \times V_{2}\right)^{\omega_{0112}} \\
& =\left\{\left(0, v_{1}, v_{1}, 0\right) \mid\left(0, v_{1}\right) \in \Lambda_{01}^{\left(-\omega_{0}\right) \oplus \omega_{1}},\left(v_{1}, 0\right) \in \Lambda_{12}^{\left(-\omega_{1}\right) \oplus \omega_{2}}\right\} \\
& \cong\left(\operatorname{im~} \Lambda_{01}\right)^{\omega_{1}} \cap\left(\operatorname{im~} \Lambda_{12}^{t}\right)^{\omega_{1}},
\end{aligned}
$$

where $\operatorname{im} \Lambda_{01}:=\pi_{V_{1}}\left(\Lambda_{01}\right) \subset V_{1}$, similarly $\operatorname{im} \Lambda_{12}^{t} \subset V_{1}$, and we used the equivalence

$$
\begin{aligned}
& \left(-\omega_{0} \oplus \omega_{1}\right)\left(\left(0, v_{1}\right),\left(\lambda_{0}, \lambda_{1}\right)\right)=0 \quad \forall\left(\lambda_{0}, \lambda_{1}\right) \in \Lambda_{01} \\
\Longleftrightarrow & \omega_{1}\left(v_{1}, \lambda_{1}\right)=0 \quad \forall \lambda_{1} \in \pi_{V_{1}}\left(\Lambda_{01}\right) .
\end{aligned}
$$

Now the two vector spaces in (5) and (6) are identified by the dualities $\operatorname{ker} \Lambda_{01}^{t}=$ $\left(\operatorname{im} \Lambda_{01}\right)^{\omega_{1}}$ and $\operatorname{ker} \Lambda_{12}=\left(\operatorname{im} \Lambda_{12}^{t}\right)^{\omega_{1}}$, which follow from the Lagrangian property of $\Lambda_{01}$ resp. $\Lambda_{12}^{t}$,

$$
\left(0, v_{1}\right) \in \Lambda_{01} \quad \Longleftrightarrow\left(0, v_{1}\right) \in \Lambda_{01}^{-\omega_{0} \oplus \omega_{1}} \Longleftrightarrow v_{1} \in\left(\mathrm{im} \Lambda_{01}\right)^{\omega_{1}} .
$$

This proves (4) and hence finishes the proof that $\pi_{02}$ is an immersion. Finally, note that an injective immersion of a compact set is automatically an embedding.

Remark 2.0.6 Suppose that the composition $L_{01} \circ L_{12}=: L_{02}$ is embedded. 
(a) By the injectivity, for every $\left(x_{0}, x_{2}\right) \in L_{02}$ there is a unique solution $x_{1} \in M_{1}$ to $\left(x_{0}, x_{1}, x_{1}, x_{2}\right) \in L_{01} \times L_{12}$. Due to the transversality assumption, this solution is given by a smooth map $\ell_{1}: L_{02} \rightarrow M_{1}$.

(b) If $L_{01}$ and $L_{12}$ are compact, oriented, and equipped with a relative spin structure, then $L_{02}$ is also compact and inherits an orientation and relative spin structure; see our paper [40]. The orientation is induced from the canonical orientation of the diagonal (see Remark 3.0.5 (b)) and the splitting

$$
T\left(M_{0} \times M_{2} \times M_{1} \times M_{1}\right)=\left(T L_{02} \times\{0\}\right) \oplus\left(\{0\} \times\left(T \Delta_{1}\right)^{\perp}\right) \oplus T\left(L_{01} \times L_{12}\right)^{\perp} .
$$

(c) If both fundamental groups $\pi_{1}\left(L_{01}\right)$ and $\pi_{1}\left(L_{12}\right)$ have torsion image in the respective ambient space, then $\pi_{1}\left(L_{02}\right)$ has torsion image in $\pi_{1}\left(M_{0} \times M_{2}\right)$. (Any loop $\gamma: S^{1} \rightarrow L_{02}$ lifts to a loop $\tilde{\gamma}: S^{1} \rightarrow L_{01} \times L_{12}$ with $\gamma=\pi_{02} \circ \tilde{\gamma}$. By assumption, some multiple cover $\tilde{\gamma}^{N}$ is the boundary of a map $\tilde{u}: D^{2} \rightarrow M_{0} \times M_{1} \times M_{1} \times M_{2}$. Hence the same cover $\gamma^{N}=\left.\left(\pi_{02} \circ \tilde{u}\right)\right|_{\partial D^{2}}$ is contractible.)

\subsection{Generalized Lagrangian correspondences}

A simple resolution of the composition problem (that geometric composition is not always defined) is given by passing to sequences of Lagrangian correspondences and composing them by concatenation. In [39] we employ these to define a symplectic category containing all smooth Lagrangian correspondences as composable morphisms, yet retaining geometric composition in cases where it is well defined.

Definition 2.1.1 Let $M, M^{\prime}$ be symplectic manifolds. A generalized Lagrangian correspondence $\underline{L}$ from $M$ to $M^{\prime}$ consists of

(a) a sequence $N_{0}, \ldots, N_{r}$ of any length $r+1 \geq 2$ of symplectic manifolds with $N_{0}=M$ and $N_{r}=M^{\prime}$,

(b) a sequence $L_{01}, \ldots, L_{(r-1) r}$ of compact Lagrangian correspondences such that $L_{(j-1) j} \subset N_{j-1}^{-} \times N_{j}$ for $j=1, \ldots, r$.

Definition 2.1.2 Let $\underline{L}$ from $M$ to $M^{\prime}$ and $\underline{L}^{\prime}$ from $M^{\prime}$ to $M^{\prime \prime}$ be two generalized Lagrangian correspondences. Then we define composition

$$
\left(\underline{L}, \underline{L}^{\prime}\right):=\left(L_{01}, \ldots, L_{(r-1) r}, L_{01}^{\prime}, \ldots, L_{\left(r^{\prime}-1\right) r^{\prime}}^{\prime}\right)
$$

as a generalized Lagrangian correspondence from $M$ to $M^{\prime \prime}$. Moreover, we define the dual

$$
\underline{L}^{t}:=\left(L_{(r-1) r}^{t}, \ldots, L_{01}^{t}\right)
$$

as a generalized Lagrangian correspondence from $M^{\prime}$ to $M$. 
We conclude this subsection by mentioning special cases of generalized Lagrangian correspondences. The first is the case $M=M^{\prime}$, which we will want to view separately as a cyclic correspondence, without fixing the "base point" $M$.

Definition 2.1.3 A cyclic generalized Lagrangian correspondence $\underline{L}$ consists of

(a) a cyclic sequence $M_{0}, M_{1}, \ldots, M_{r}, M_{r+1}=M_{0}$ of symplectic manifolds of any length $r+1 \geq 1$,

(b) a sequence $L_{01}, \ldots, L_{r(r+1)}$ of compact Lagrangian correspondences such that $L_{j(j+1)} \subset M_{j}^{-} \times M_{j+1}$ for $j=0, \ldots, r$.

The second special case is $M=\{\mathrm{pt}\}$, which generalizes the concept of Lagrangian submanifolds. Namely, note that any Lagrangian submanifold $L \subset M^{\prime}$ can be viewed as correspondence $L \subset\{\mathrm{pt}\}^{-} \times M^{\prime}$.

Definition 2.1.4 Let $M^{\prime}$ be a symplectic manifold. A generalized Lagrangian submanifold $\underline{L}$ of $M^{\prime}$ is a generalized Lagrangian correspondence from a point $M=\{\mathrm{pt}\}$ to $M^{\prime}$. That is, $\underline{L}$ consists of

(a) a sequence $N_{-r}, \ldots, N_{0}$ of any length $r \geq 0$ of symplectic manifolds with $N_{-r}=\{\mathrm{pt}\}$ a point and $N_{0}=M^{\prime}$,

(b) a sequence $L_{(-r)(-r+1)}, \ldots, L_{(-1) 0}$ of compact Lagrangian correspondences such that $L_{(i-1) i} \subset N_{i-1}^{-} \times N_{i}$.

\section{Gradings}

The purpose of this section is to review the theory of graded Lagrangians and extend it to generalized Lagrangian correspondences. It can be skipped at first reading.

Following Kontsevich and Seidel [31] one can define graded Lagrangian subspaces as follows. Let $V$ be a symplectic vector space and let $\operatorname{Lag}(V)$ be the Lagrangian Grassmannian of $V$. An $N$-fold Maslov covering for $V$ is a $\mathbb{Z}_{N}$-covering $\operatorname{Lag}^{N}(V) \rightarrow$ $\operatorname{Lag}(V)$ associated to the Maslov class in $\operatorname{Hom}\left(\pi_{1}(\operatorname{Lag}(V)), \mathbb{Z}\right)$. (More precisely, the $\bmod N$ reduction of the Maslov class defines a representation $\pi_{1}(\operatorname{Lag}(V)) \rightarrow \mathbb{Z}_{N}$. Let $\widetilde{\operatorname{Lag}}(V)$ be the universal cover of $\operatorname{Lag}(V)$, then the $N$-fold Maslov covering associated to the given representation is the associated bundle $\widetilde{\operatorname{Lag}}(V) \times_{\pi_{1}(\operatorname{Lag}(V))} \mathbb{Z}_{N} \rightarrow \operatorname{Lag}(V)$.) A grading of a Lagrangian subspace $\Lambda \in \operatorname{Lag}(V)$ is a lift to $\widetilde{\Lambda} \in \operatorname{Lag}^{N}(V)$. 
Remark 3.0.5 (a) For any basepoint $\Lambda_{0} \in \operatorname{Lag}(V)$ we obtain an $N$-fold Maslov cover $\operatorname{Lag}^{N}\left(V, \Lambda_{0}\right)$ given as the homotopy classes of paths $\widetilde{\Lambda}:[0,1] \rightarrow \operatorname{Lag}(V)$ with base point $\tilde{\Lambda}(0)=\Lambda_{0}$, modulo loops whose Maslov index is a multiple of $N$. The covering is $\tilde{\Lambda} \mapsto \tilde{\Lambda}(1)$. The base point has a canonical grading given by the constant path $\tilde{\Lambda}_{0} \equiv \Lambda_{0}$. Any path between basepoints $\Lambda_{0}, \Lambda_{0}^{\prime}$ induces an identification $\operatorname{Lag}^{N}\left(V, \Lambda_{0}\right) \rightarrow \operatorname{Lag}^{N}\left(V, \Lambda_{0}^{\prime}\right)$.

(b) For the diagonal $\Delta \subset V^{-} \times V$ we fix a canonical grading and orientation as follows. (This choice is made in order to obtain the degree identity in Lemma 3.0.12 (d).) We identify the Maslov coverings $\operatorname{Lag}^{N}\left(V^{-} \times V, \Lambda^{-} \times \Lambda\right)$ and $\operatorname{Lag}^{N}\left(V^{-} \times V, \Delta\right)$ by concatenation of the paths ${ }^{2}$

$$
\left(e^{J t} \Lambda^{-} \times \Lambda\right)_{t \in[0, \pi / 2]}, \quad(\{(t x+J y, x+t J y) \mid x, y \in \Lambda\})_{t \in[0,1]},
$$

for $J \in \operatorname{End}(V)$ an $\omega$-compatible complex structure on $V$ (ie $J^{2}=-\operatorname{Id}$ and $\omega(\cdot, J \cdot$ ) is symmetric and positive definite). In particular, this induces the canonical grading on the diagonal $\Delta$ with respect to any Maslov covering $\operatorname{Lag}^{N}\left(V^{-} \times V, \Lambda^{-} \times \Lambda\right)$, by continuation. Any identification $\operatorname{Lag}^{N}\left(V^{-} \times V, \Lambda_{0}^{-} \times \Lambda_{0}\right) \rightarrow \operatorname{Lag}^{N}\left(V^{-} \times V, \Lambda_{1}^{-} \times \Lambda_{1}\right)$ induced by a path in $\operatorname{Lag}^{N}(V)$ maps the graded diagonal to the graded diagonal, since the product $\gamma^{-} \times \gamma$ of any loop $\gamma: S^{1} \rightarrow \operatorname{Lag}(V)$ has Maslov index 0. Similarly, we define a canonical orientation on $\Delta$ by choosing any orientation on $\Lambda$, giving the product $\Lambda^{-} \times \Lambda$ the product orientation (which is well defined), and extending the orientation over the path (7). This is related to the orientation induced by projection of the diagonal on the second factor by a sign $(-1)^{n(n-1) / 2}$, where $\operatorname{dim}(M)=2 n$.

Let $M$ be a symplectic manifold and let $\operatorname{Lag}(M) \rightarrow M$ be the fiber bundle whose fiber over $m \in M$ is the space $\operatorname{Lag}\left(T_{m} M\right)$ of Lagrangian subspaces of $T_{m} M$. An $N$-fold Maslov covering of $M$ is an $N$-fold cover $\operatorname{Lag}^{N}(M) \rightarrow \operatorname{Lag}(M)$ whose restriction to each fiber is an $N$-fold Maslov covering $\operatorname{Lag}^{N}\left(T_{m} M\right) \rightarrow \operatorname{Lag}\left(T_{m} M\right)$. Any choice of Maslov cover for $\mathbb{R}^{2 n}$ induces a one-to-one correspondence between $N$-fold Maslov covers of $M$ and $\operatorname{Sp}^{N}(2 n)$-structures on $M$. Here $2 n=\operatorname{dim} M$ and $\operatorname{Sp}^{N}(2 n)$ is the $N$-fold covering group of $\operatorname{Sp}(2 n)$ associated to the $\bmod N$ reduction of the Maslov class in $\operatorname{Hom}\left(\pi_{1}(\operatorname{Sp}(2 n)), \mathbb{Z}_{N}\right)$. (Explicitly, this is realized by using the identity as base point.) An $\operatorname{Sp}^{N}(2 n)$-structure on $M$ is an $\operatorname{Sp}^{N}(2 n)$-bundle $\operatorname{Fr}^{N}(M) \rightarrow M$ together with an isomorphism $\operatorname{Fr}^{N}(M) \times_{\mathrm{Sp}^{N}(2 n)} \operatorname{Sp}(2 n) \simeq \operatorname{Fr}(M)$ to the symplectic frame bundle of $M$. It induces the $N$-fold Maslov covering

$$
\stackrel{N}{\operatorname{Lag}(M)}=\stackrel{N}{\operatorname{Fr}}(M) \times_{\mathrm{Sp}^{N}(2 n)} \stackrel{N}{\operatorname{Lag}\left(\mathbb{R}^{2 n}\right) .}
$$

2 The first path arises from the canonical path between $\operatorname{Id}$ and $J$ in $\operatorname{Symp}(V)$. The second path can be understood as the graphs of the symplectomorphisms $\left(t^{-1} \mathrm{Id}\right) \times(t \mathrm{Id})$ on $V \cong \Lambda \times J \Lambda$. For $t \rightarrow 0$ this graph converges to the split Lagrangian $J \Lambda \times \Lambda \subset V^{-} \times V$; for $t=1$ the graph is the diagonal. 
The notions of duals, disjoint union, and Cartesian product extend naturally to the graded setting as follows. The dual $\operatorname{Lag}^{N}\left(M^{-}\right)$of a Maslov covering $\operatorname{Lag}^{N}(M) \rightarrow \operatorname{Lag}(M)$ is the same space with the inverted $\mathbb{Z}_{N}$-action. We denote this identification by

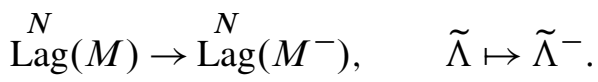

For $\operatorname{Sp}^{N}$-structures $\operatorname{Fr}^{N}\left(M_{0}\right)$ and $\operatorname{Fr}^{N}\left(M_{1}\right)$ the embedding

$$
\stackrel{N}{\mathrm{Sp}}\left(2 n_{0}\right) \times_{\mathbb{Z}_{N}} \stackrel{N}{\mathrm{Sp}}\left(2 n_{1}\right) \rightarrow \stackrel{N}{\mathrm{Sp}}\left(2 n_{0}+2 n_{1}\right)
$$

induces an $\mathrm{Sp}^{N}\left(2 n_{0}+2 n_{1}\right)$-structure $\mathrm{Fr}^{N}\left(M_{0} \times M_{1}\right)$ on the product and an equivariant map

$$
\stackrel{N}{\operatorname{Fr}}\left(M_{0}\right) \times \stackrel{N}{\operatorname{Fr}}\left(M_{1}\right) \rightarrow \stackrel{N}{\operatorname{Fr}}\left(M_{0} \times M_{1}\right)
$$

covering the inclusion $\operatorname{Fr}\left(M_{0}\right) \times \operatorname{Fr}\left(M_{1}\right) \rightarrow \operatorname{Fr}\left(M_{0} \times M_{1}\right)$. The corresponding product of $N$-fold Maslov covers on $M_{0} \times M_{1}$ is the $N$-fold Maslov covering

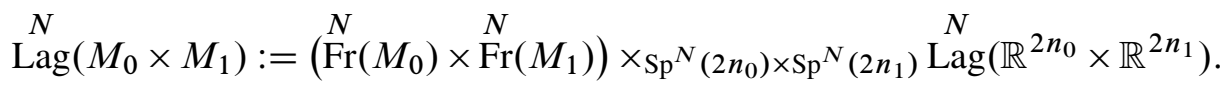

Combining this product with the dual yields a Maslov covering for $M_{0}^{-} \times M_{1}$ which we can identify with

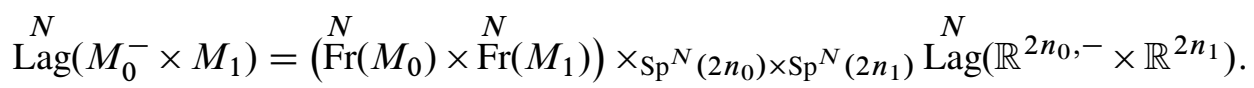

Finally, the inclusion $\operatorname{Lag}\left(M_{0}\right) \times \operatorname{Lag}\left(M_{1}\right) \rightarrow \operatorname{Lag}\left(M_{0} \times M_{1}\right)$ lifts to a map

$$
\stackrel{N}{\operatorname{Lag}}\left(M_{0}\right) \times \stackrel{N}{\operatorname{Lag}}\left(M_{1}\right) \rightarrow \stackrel{N}{\operatorname{Lag}}\left(M_{0} \times M_{1}\right), \quad\left(\widetilde{L}_{0}, \widetilde{L}_{1}\right) \mapsto \widetilde{L}_{0} \times{ }^{N} \widetilde{L}_{1}
$$

with fiber $\mathbb{Z}_{N}$. It is defined by combining the product (9) with the basic product of the linear Maslov cover $\operatorname{Lag}^{N}\left(\mathbb{R}^{2 n_{0}}\right) \times \operatorname{Lag}^{N}\left(\mathbb{R}^{2 n_{1}}\right) \rightarrow \operatorname{Lag}^{N}\left(\mathbb{R}^{2 n_{0}} \times \mathbb{R}^{2 n_{1}}\right)$.

Definition 3.0.6 (a) Let $M_{0}, M_{1}$ be two symplectic manifolds equipped with $N-$ fold Maslov covers and let $\phi: M_{0} \rightarrow M_{1}$ be a symplectomorphisms. A grading of $\phi$ is a lift of the canonical isomorphism $\operatorname{Lag}\left(M_{0}\right) \rightarrow \operatorname{Lag}\left(M_{1}\right)$ to an isomorphism $\phi^{N}: \operatorname{Lag}^{N}\left(M_{0}\right) \rightarrow \operatorname{Lag}^{N}\left(M_{1}\right)$, or equivalently, a lift of the canonical isomorphism $\operatorname{Fr}\left(M_{0}\right) \rightarrow \operatorname{Fr}\left(M_{1}\right)$ of symplectic frame bundles to an isomorphism $\operatorname{Fr}^{N}\left(M_{0}\right) \rightarrow$ $\operatorname{Fr}^{N}\left(M_{1}\right)$.

(b) Let $L \subset M$ be a Lagrangian submanifold and $M$ be equipped with an $N$-fold Maslov cover. A grading of $L$ is a lift $\sigma_{L}^{N}: L \rightarrow \operatorname{Lag}^{N}(M)$ of the canonical section $\sigma_{L}: L \rightarrow \operatorname{Lag}(M)$. 
Remark 3.0.7 (a) The set of graded symplectomorphisms forms a group under composition. In particular, the identity on $M$ has a canonical grading, given by the identity on $\operatorname{Lag}^{N}(M)$.

(b) Given a one-parameter family $\phi_{t}$ of symplectomorphisms with $\phi_{0}=\operatorname{Id}_{M}$, we obtain a grading of $\phi_{t}$ by continuity.

(c) Any choice of grading on the diagonal $\widetilde{\Delta} \in \operatorname{Lag}^{N}\left(\mathbb{R}^{2 n,-} \times \mathbb{R}^{2 n}\right)$ induces a bijection between gradings of a symplectomorphism $\phi: M_{0} \rightarrow M_{1}$ and gradings of its graph $\operatorname{graph}(\phi) \subset M_{0}^{-} \times M_{1}$ with respect to the induced Maslov cover $\operatorname{Lag}^{N}\left(M_{0}^{-} \times M_{1}\right)$. Indeed, the graph of the grading, $\left.\operatorname{graph}\left(\phi^{N}\right) \subset\left(\operatorname{Fr}^{N}\left(M_{0}\right) \times \operatorname{Fr}^{N}\left(M_{1}\right)\right)\right|_{\operatorname{graph}(\phi)}$ is a principal bundle over $\operatorname{graph}(\phi)$ with structure group $\operatorname{Sp}^{N}(2 n), 2 n=\operatorname{dim} M_{0}=$ $\operatorname{dim} M_{1}$. The graded diagonal descends under the associated fiber bundle construction with $\operatorname{graph}\left(\phi^{N}\right)$ to a section of $\left.\operatorname{Lag}^{N}\left(M_{0}^{-} \times M_{1}\right)\right|_{\operatorname{graph}(\phi)} \operatorname{lifting} \operatorname{graph}(\phi)$. Moreover, this construction is equivariant for the transitive action of $H^{0}\left(M_{0}, \mathbb{Z}_{N}\right)$ on both the set of gradings of $\phi$ and the set of gradings of $\operatorname{graph}(\phi)$.

We will refer to this as the canonical bijection when using the canonical grading $\widetilde{\Delta} \in \operatorname{Lag}^{N}\left(\mathbb{R}^{2 n,-} \times \mathbb{R}^{2 n}\right)$ in Remark 3.0.5. In particular, the diagonal in $M^{-} \times M$ has a canonical grading induced by the canonical bijection from the canonical grading of the identity on $M$.

(d) Any grading $\sigma_{L}^{N}$ of a Lagrangian submanifold $L \subset M$ induces a grading of $L \subset M^{-}$via the diffeomorphism $\operatorname{Lag}^{N}\left(M^{-}\right) \rightarrow \operatorname{Lag}^{N}(M)$.

(e) Given graded Lagrangian submanifolds $L_{0} \subset M_{0}, L_{1} \subset M_{1}$, the product submanifold $L_{0} \times L_{1} \subset M_{0} \times M_{1}$ inherits a grading from (10).

(f) Given a graded symplectomorphism $\phi: M_{0} \rightarrow M_{1}$ and a graded Lagrangian submanifold $L \subset M_{0}$, the image $\phi(L) \subset M_{1}$ inherits a grading by composition $\sigma_{\phi(L)}^{N}=\phi^{N} \circ \sigma_{L}^{N}$.

Example 3.0.8 (a) Let $\operatorname{Lag}^{2}(M)$ be the bundle whose fiber over $m$ is the space of oriented Lagrangian subspaces of $T_{m} M$. Then $\operatorname{Lag}^{2}(M) \rightarrow \operatorname{Lag}(M)$ is a 2 -fold Maslov covering. A $\operatorname{Lag}^{2}(M)$-grading of a Lagrangian $L \subset M$ is equivalent to an orientation on $L$.

(b) By [31, Section 2], any symplectic manifold $M$ with $H^{1}(M)=0$ and minimal Chern number $N_{M}$ admits an $N$-fold Maslov covering $\operatorname{Lag}^{N}(M)$ if and only if $N$ divides $2 N_{M}$. Any Lagrangian with minimal Maslov number $N_{L}$ admits a $\operatorname{Lag}^{N}(M)-$ grading if and only if $N$ divides $N_{L}$. In particular, if $H^{1}(M)=0$ and $L$ is simply connected, then $N_{L}=2 N_{M}$ and $L$ admits a $\operatorname{Lag}^{2 N_{M}}(M)$ grading. 
(c) Suppose that $[\omega]$ is integral, $[\omega]=(1 / l) c_{1}(T M)$, and $\mathcal{L}$ is a line bundle with connection $\nabla$ and curvature $\operatorname{curv}(\nabla)=(2 \pi / i) \omega$. This induces a $2 l$-fold Maslov cover $\operatorname{Lag}^{2 l}(M) \rightarrow \operatorname{Lag}(M)$; see Seidel [31, Section 2b]. Let $L \subset M$ be a BohrSommerfeld monotone Lagrangian as in Remark 4.1.4. A grading of $L$ is equivalent to a choice of (not necessarily horizontal) section of $\mathcal{L} \mid L$ whose $l$-th tensor power is $\phi_{L}^{\mathcal{K}}$; that is, a choice of the section $\exp (2 \pi i \psi) \phi_{L}^{\mathcal{L}}$ in (19).

Definition 3.0.9 Let $\Lambda_{0}, \Lambda_{1} \subset V$ be a transverse pair of Lagrangian subspaces in a symplectic vector space $V$ and let $\tilde{\Lambda}_{0}, \tilde{\Lambda}_{1} \in \operatorname{Lag}^{N}(V)$ be gradings. The degree $d\left(\widetilde{\Lambda}_{0}, \tilde{\Lambda}_{1}\right) \in \mathbb{Z}_{N}$ is defined as follows. Let $\tilde{\gamma}_{0}, \tilde{\gamma}_{1}:[0,1] \rightarrow \operatorname{Lag}^{N}(V)$ be paths with common starting point $\tilde{\gamma}_{0}(0)=\tilde{\gamma}_{1}(0)$ and end points $\tilde{\gamma}_{j}(1)=\tilde{\Lambda}_{j}$. Let $\gamma_{j}:[0,1] \rightarrow$ $\operatorname{Lag}(V)$ denote their image under the projection $\operatorname{Lag}^{N}(V) \rightarrow \operatorname{Lag}(V)$ and define

$$
d\left(\tilde{\Lambda}_{0}, \tilde{\Lambda}_{1}\right):=\frac{1}{2} \operatorname{dim}\left(\Lambda_{0}\right)+I\left(\gamma_{0}, \gamma_{1}\right) \bmod N,
$$

where $I\left(\gamma_{0}, \gamma_{1}\right)$ denotes the Maslov index for the pair of paths as in [36; 27].

Let us recall from [27] that the Maslov index for a pair of paths with regular crossings (in particular with a finite set of crossings $\left.\mathcal{C}:=\left\{s \in[0,1] \mid \gamma_{0}(s) \cap \gamma_{1}(s) \neq\{0\}\right\}\right)$ is given by the sum of crossing numbers with the endpoints weighted by $1 / 2$,

$$
I\left(\gamma_{0}, \gamma_{1}\right)=\frac{1}{2} \sum_{s \in \mathcal{C} \cap\{0,1\}} \operatorname{sign}\left(\Gamma\left(\gamma_{0}, \gamma_{1}, s\right)\right)+\sum_{s \in \mathcal{C} \cap(0,1)} \operatorname{sign}\left(\Gamma\left(\gamma_{0}, \gamma_{1}, s\right)\right) .
$$

Each crossing operator $\Gamma\left(\gamma_{0}, \gamma_{1}, s\right)$ is defined on $v \in \gamma_{0}(s) \cap \gamma_{1}(s)$ by fixing Lagrangian complements $\gamma_{0}(s)^{c}, \gamma_{1}(s)^{c}$ of $\gamma_{0}(s), \gamma_{1}(s)$ and setting

$$
\Gamma\left(\gamma_{0}, \gamma_{1}, s\right) v=\left.\frac{d}{d t}\right|_{t=0} \omega\left(v, w(t)-w^{\prime}(t)\right)
$$

where $w(t) \in \gamma_{0}(s)^{c}$ such that $v+w(t) \in \gamma_{0}(s+t)$ and $w^{\prime}(t) \in \gamma_{1}(s)^{c}$ such that $v+w^{\prime}(s+t) \in \gamma_{1}(s)$.

Remark 3.0.10 The degree can alternatively be defined by fixing $\tilde{\gamma}_{0} \equiv \tilde{\Lambda}_{0}$ and choosing a path $\tilde{\gamma}:[0,1] \rightarrow \operatorname{Lag}^{N}(V)$ from $\tilde{\gamma}(0)=\tilde{\Lambda}_{0}$ to $\tilde{\gamma}(1)=\widetilde{\Lambda}_{1}$ such that the crossing form $\Gamma\left(\gamma, \Lambda_{0}, 0\right)$ of the underlying path $\gamma:[0,1] \rightarrow \operatorname{Lag}(V)$ is positive definite at $s=0$. Then the degree

$d\left(\tilde{\Lambda}_{0}, \tilde{\Lambda}_{1}\right)=\frac{\operatorname{dim} \Lambda_{0}}{2}+I\left(\Lambda_{0}, \gamma\right)=-\sum_{s \in(0,1)} \operatorname{sign}\left(\Gamma\left(\gamma, \Lambda_{0}, s\right)\right)=-I^{\prime}\left(\gamma, \Lambda_{0}\right) \quad \bmod N$

is given by the Maslov index $I^{\prime}$ of $\left.\gamma\right|_{(0,1)}$ (not counting the endpoints) relative to $\Lambda_{0}$. Equivalently, we have

$$
d\left(\tilde{\Lambda}_{0}, \tilde{\Lambda}_{1}\right)=I^{\prime}\left(\gamma^{-1}, \Lambda_{0}\right) \bmod N
$$


for the reversed path $\gamma^{-1}:[0,1] \rightarrow \operatorname{Lag}(V)$ from $\gamma^{-1}(0)=\Lambda_{1}$ to $\gamma^{-1}(1)=\Lambda_{0}$ such that the crossing form $\Gamma\left(\gamma^{-1}, \Lambda_{0}, 1\right)$ is negative definite at $s=1$.

Lemma 3.0.11 (Index theorem for once-punctured disks) Let $\Lambda_{0}, \Lambda_{1} \subset V$ be a transverse pair of Lagrangian subspaces with gradings $\widetilde{\Lambda}_{0}, \widetilde{\Lambda}_{1} \in \operatorname{Lag}^{N}(V)$. Then for any smooth path of graded Lagrangian subspaces $\tilde{\Lambda}:[0,1] \rightarrow \operatorname{Lag}^{N}(V)$ with endpoints $\tilde{\Lambda}(j)=\tilde{\Lambda}_{j}, j=0,1$ we have

$$
d\left(\tilde{\Lambda}_{0}, \tilde{\Lambda}_{1}\right)=\operatorname{Ind}\left(D_{V, \Lambda}\right) \bmod N .
$$

Here $D_{V, \Lambda}$ is any Cauchy-Riemann operator in $V$ on the disk $D$ with one outgoing strip-like end $(0, \infty) \times[0,1] \hookrightarrow D$ and with boundary conditions given by $\Lambda$ (the projection of $\tilde{\Lambda}$ to $\operatorname{Lag}(V)$ ) such that $\Lambda(j)=\Lambda_{j}$ is the boundary condition over the boundary components $(0, \infty) \times\{j\}, j=0,1$ of the end.

Proof It suffices to prove the index identity for a fixed path $\tilde{\Lambda}$. Indeed, if $\widetilde{\Lambda}^{\prime}$ is any other path with the same endpoints then we have $\operatorname{Ind}\left(D_{V, \Lambda}\right)-\operatorname{Ind}\left(D_{V, \Lambda^{\prime}}\right)=$ $\operatorname{Ind}\left(D_{V, \Lambda}\right)+\operatorname{Ind}\left(D_{V,-\Lambda^{\prime}}\right)=\operatorname{Ind}\left(D_{V, \Lambda \#\left(-\Lambda^{\prime}\right)}\right)$ by gluing. Here the last CauchyRiemann operator is defined on the disk with no punctures and with boundary conditions given by the loop $\Lambda \#\left(-\Lambda^{\prime}\right)$. Since the loop lifts to a loop $\widetilde{\Lambda} \#\left(-\widetilde{\Lambda}^{\prime}\right)$ in $\operatorname{Lag}^{N}(V)$, its Maslov index (and thus index) is 0 modulo $N$.

By Remark 3.0.10, the degree can be defined by a path $\tilde{\Lambda}$ from $\tilde{\Lambda}_{1}$ to $\widetilde{\Lambda}_{0}$ whose projection $\Lambda$ has negative definite crossing form at $s=1$. The sum of crossing numbers in $d\left(\widetilde{\Lambda}_{0}, \tilde{\Lambda}_{1}\right)=\sum_{s \in(0,1)} \operatorname{sign}\left(\Gamma\left(\Lambda, \Lambda_{0}, s\right)\right)$ is the Maslov index $I_{H}(\Lambda)$ in [33, Lemma 11.11] and hence equals to the Fredholm index $\operatorname{Ind}\left(D_{V, \Lambda}\right)$ over the half space, or the conformally equivalent disk with strip-like end. This conformal isomorphism takes the boundary ends $(-\infty,-1)$ resp. $(1, \infty)$ in the half space $\{\operatorname{Im} z \geq 0\}$ (over which $\Lambda$ equals to $\Lambda_{1}$ resp. $\left.\Lambda_{0}\right)$ to $\{1\} \times(1, \infty)$ resp. $\{0\} \times(1, \infty)$ in the strip-like end.

Lemma 3.0.12 The degree map satisfies the following properties.

(a) (Additivity) If $V=V^{\prime} \times V^{\prime \prime}$ then

$$
d\left(\tilde{\Lambda}_{0}^{\prime} \times{ }^{N} \tilde{\Lambda}_{0}^{\prime \prime}, \tilde{\Lambda}_{1}^{\prime} \times^{N} \tilde{\Lambda}_{1}^{\prime \prime}\right)=d\left(\tilde{\Lambda}_{0}^{\prime}, \tilde{\Lambda}_{1}^{\prime}\right)+d\left(\tilde{\Lambda}_{0}^{\prime \prime}, \tilde{\Lambda}_{1}^{\prime \prime}\right)
$$

for $\widetilde{\Lambda}_{j}^{\prime}, \tilde{\Lambda}_{j}^{\prime \prime}$ graded Lagrangian subspaces in $V^{\prime}, V^{\prime \prime}$ respectively, $j=0,1$.

(b) (Multiplicativity) For $\tilde{\Lambda}_{0}, \widetilde{\Lambda}_{1}$ graded Lagrangian subspaces and any $c \in \mathbb{Z}_{N}$

$$
d\left(\tilde{\Lambda}_{0}, c \cdot \tilde{\Lambda}_{1}\right)=c+d\left(\tilde{\Lambda}_{0}, \tilde{\Lambda}_{1}\right) .
$$

(c) (Skewsymmetry) For $\tilde{\Lambda}_{0}, \tilde{\Lambda}_{1}$ graded Lagrangian subspaces

$$
d\left(\tilde{\Lambda}_{0}, \tilde{\Lambda}_{1}\right)+d\left(\tilde{\Lambda}_{1}, \tilde{\Lambda}_{0}\right)=\operatorname{dim} \Lambda_{0}=d\left(\tilde{\Lambda}_{0}, \tilde{\Lambda}_{1}\right)+d\left(\tilde{\Lambda}_{0}^{-}, \tilde{\Lambda}_{1}^{-}\right) .
$$


(d) (Diagonal) For a transverse pair $\tilde{\Lambda}_{0}, \widetilde{\Lambda}_{1}$ of graded Lagrangian subspaces in $V$ and $\widetilde{\Delta}$ the canonically graded diagonal in $V^{-} \times V$

$$
d\left(\widetilde{\Delta}, \tilde{\Lambda}_{0}^{-} \times^{N} \tilde{\Lambda}_{1}\right)=d\left(\tilde{\Lambda}_{0}, \tilde{\Lambda}_{1}\right) .
$$

Proof The first three properties are standard; see Seidel [31, Section 2d]. We prove the diagonal property to make sure all our sign conventions match up. For that purpose we fix $\widetilde{L} \in \operatorname{Lag}^{N}(V)$ and choose the following paths $\tilde{\gamma}_{\text {.. }}$ of graded Lagrangian subspaces

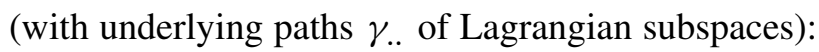

- $\tilde{\gamma}_{0}:[-1,1] \rightarrow \operatorname{Lag}^{N}(V)$ from $\tilde{\gamma}_{0}(-1)=\widetilde{L}$ to $\tilde{\gamma}_{0}(1)=\tilde{\Lambda}_{0}$ such that $\left.\tilde{\gamma}_{0}\right|_{[-1,0]} \equiv \tilde{L}$,

- $\tilde{\gamma}_{1}:[-1,1] \rightarrow \operatorname{Lag}^{N}(V)$ from $\tilde{\gamma}_{1}(-1)=\widetilde{L}$ to $\tilde{\gamma}_{1}(1)=\tilde{\Lambda}_{1}$, such that $\left.\gamma_{1}\right|_{[-1 / 2,0]} \equiv$ $J L \pitchfork L$ and $\left.\gamma_{1}\right|_{[-1,-1 / 2]}$ is a smoothing of $t \mapsto e^{\pi(1+t) J} L$.

- $\tilde{\gamma}:[-1,1] \rightarrow \operatorname{Lag}^{N}\left(V^{-} \times V\right)$ starting with $\left.\tilde{\gamma}\right|_{[-1,-1 / 2]}=\left.\left(\tilde{\gamma}_{1}^{-} \times{ }^{N} \tilde{\gamma}_{0}\right)\right|_{[-1,-1 / 2]}$, ending at $\left.\tilde{\gamma}\right|_{[0,1]} \equiv \tilde{\Delta}$, and such that $\left.\gamma\right|_{[-1 / 2,0]}$ is a smoothing of $t \mapsto$ $\{((2 t+1) x+J y, x+(2 t+1) J y) \mid x, y \in L\}$. (The lift to graded subspaces matches up since $\left.\gamma\right|_{[-1,0]}$ is exactly the path of (7) which defines $\widetilde{\Delta}$ by connecting it to $\widetilde{L}^{-} \times \widetilde{L}$.)

Note that we have $\left.I\left(\gamma_{0}, \gamma_{1}\right)\right|_{[-1,0]}=-\frac{1}{2} \operatorname{dim} \Lambda_{0}$ and

$$
\left.I\left(\gamma, \gamma_{0}^{-} \times \gamma_{1}\right)\right|_{[-1,0]}=\left.I\left(\gamma_{1}^{-}, \gamma_{0}^{-}\right)\right|_{[-1,0]}+\left.I\left(\gamma_{0}, \gamma_{1}\right)\right|_{[-1,0]}=-\operatorname{dim} \Lambda_{0}
$$

since $\left.\gamma\right|_{[-1 / 2,0]}$ is transverse to $L^{-} \times J L$. With these preparations we can calculate

$$
\begin{aligned}
d\left(\tilde{\Lambda}_{0}, \tilde{\Lambda}_{1}\right) & =\frac{1}{2} \operatorname{dim} \Lambda_{0}+I\left(\gamma_{0}, \gamma_{1}\right)=\left.I\left(\gamma_{0}, \gamma_{1}\right)\right|_{[0,1]} \\
& =\left.I\left(\Delta, \gamma_{0}^{-} \times \gamma_{1}\right)\right|_{[0,1]} \\
& =\operatorname{dim} \Lambda_{0}+I\left(\gamma, \gamma_{0}^{-} \times \gamma_{1}\right)=d\left(\widetilde{\Delta}, \tilde{\Lambda}_{0}^{-} \times{ }^{N} \tilde{\Lambda}_{1}\right) .
\end{aligned}
$$

Here the identity of the Maslov indices over the interval $[0,1]$ follows from identifying the intersections $K(s):=\gamma_{0} \cap \gamma_{1} \cong \Delta \cap\left(\gamma_{0}^{-} \times \gamma_{1}\right)$ and the crossing forms $\Gamma(s), \hat{\Gamma}(s): K(s) \rightarrow \mathbb{R}$ at regular crossings $s \in[0,1]$ (after a homotopy of the paths to regular crossings). Fix Lagrangian complements $\gamma_{0}(s)^{c}$ and $\gamma_{1}(s)^{c}$, then for $v \in K(s)$ pick $w_{i}(t) \in \gamma_{i}(s)^{c}$ such that $v+w_{i}(t) \in \gamma_{i}(s+t)$. For the corresponding vector $\hat{v}=(v, v) \in \Delta \cap\left(\gamma_{0}^{-} \times \gamma_{1}\right)$ we can pick $\widehat{w}(t)=(0,0) \in \Delta^{c}$ satisfying $\widehat{v}+\widehat{w}(t) \in \Delta$ and $\widehat{w}^{\prime}(t)=\left(w_{0}, w_{1}\right) \in \gamma_{0}(s)^{c} \times \gamma_{1}(s)^{c}$ satisfying $\widehat{v}+\widehat{w}^{\prime}(t) \in\left(\gamma_{0} \times \gamma_{1}\right)(s+t)$ to identify the crossing forms

$$
\begin{aligned}
\widehat{\Gamma}(s) \widehat{v} & =\left.\frac{d}{d t}\right|_{t=0}(-\omega \oplus \omega)\left(\widehat{v}, \widehat{w}(t)-\widehat{w}^{\prime}(t)\right) \\
& =\left.\frac{d}{d t}\right|_{t=0}\left(-\omega\left(v,-w_{0}(t)\right)+\omega\left(v,-w_{1}(t)\right)\right) \\
& =\left.\frac{d}{d t}\right|_{t=0} \omega\left(v, w_{0}(t)-w_{1}(t)\right)=\Gamma(s) v .
\end{aligned}
$$


If $L_{0}, L_{1} \subset M$ are $\operatorname{Lag}^{N}(M)$-graded Lagrangians and intersect transversally then one obtains a degree map

$$
\mathcal{I}\left(L_{0}, L_{1}\right):=L_{0} \cap L_{1} \rightarrow \mathbb{Z}_{N}, \quad x \mapsto|x|:=d\left(\sigma_{L_{0}}^{N}(x), \sigma_{L_{1}}^{N}(x)\right) .
$$

More generally, if $L_{0}, L_{1}$ do not necessarily intersect transversally, then we can pick a Hamiltonian perturbation $H:[0,1] \times M \rightarrow \mathbb{R}$ such that its time 1 flow $\phi_{1}: M \rightarrow M$ achieves transversality $\phi_{1}\left(L_{0}\right) \pitchfork L_{1}$. Then the Hamiltonian isotopy and the grading on $L_{0}$ induce a grading on $\phi_{1}\left(L_{0}\right)$, which is transverse to $L_{1}$. The degree map is then defined on the perturbed intersection points, $d: \mathcal{I}\left(L_{0}, L_{1}\right):=\phi_{1}\left(L_{0}\right) \cap L_{1} \rightarrow \mathbb{Z}_{N}$.

\subsection{Graded generalized Lagrangian correspondences}

In this section we extend the grading and degree constructions to generalized Lagrangian correspondences and discuss their behaviour under geometric composition and insertion of the diagonal.

Definition 3.1.1 Let $M$ and $M^{\prime}$ be symplectic manifolds equipped with $N$-fold Maslov coverings. Let $\underline{L}=\left(L_{01}, \ldots, L_{(r-1) r}\right)$ be a generalized Lagrangian correspondence from $M$ to $M^{\prime}$ (ie $L_{(j-1) j} \subset M_{j-1}^{-} \times M_{j}$ for a sequence $M=M_{1}, \ldots, M_{r}=$ $M^{\prime}$ of symplectic manifolds). A grading on $\underline{L}$ consists of a collection of $N$-fold Maslov covers $\operatorname{Lag}^{N}\left(M_{j}\right) \rightarrow M_{j}$ and gradings of the Lagrangian correspondences $L_{(j-1) j}$ with respect to $\operatorname{Lag}^{N}\left(M_{j-1}^{-} \times M_{j}\right)$, where the Maslov covers on $M_{1}=M$ and $M_{r}=M^{\prime}$ are the fixed ones.

A pair of graded generalized Lagrangian correspondences $\underline{L}_{1}$ and $\underline{L}_{2}$ from $M$ to $M^{\prime}$ (with fixed Maslov coverings) defines a cyclic Lagrangian correspondence $\underline{L}_{1} \#\left(\underline{L}_{2}\right)^{t}$, which is graded in the following sense.

Definition 3.1.2 Let $\underline{L}=\left(L_{01}, \ldots, L_{r(r+1)}\right)$ be a cyclic generalized Lagrangian correspondence (ie $L_{j(j+1)} \subset M_{j}^{-} \times M_{j+1}$ for a cyclic sequence $M_{0}, M_{1}, \ldots, M_{r+1}=$ $M_{0}$ of symplectic manifolds). An $N$-grading on $\underline{L}$ consists of a collection of $N$-fold Maslov covers $\operatorname{Lag}^{N}\left(M_{j}\right) \rightarrow M_{j}$ and gradings of the Lagrangian correspondences $L_{j(j+1)}$ with respect to $\operatorname{Lag}^{N}\left(M_{j}^{-} \times M_{j+1}\right)$.

In the following, we will consider a cyclic generalized Lagrangian correspondence $\underline{L}$ and assume that it intersects the generalized diagonal transversally, ie

$$
\left(L_{01} \times L_{12} \times \cdots \times L_{r(r+1)}\right) \pitchfork\left(\Delta_{M_{0}}^{-} \times \Delta_{M_{1}}^{-} \times \cdots \times \Delta_{M_{r}}^{-}\right)^{T},
$$

where $\Delta_{M}^{-} \subset M \times M^{-}$is the (dual of the) diagonal and $M_{0} \times M_{0}^{-} \times M_{1} \times \cdots \times M_{r}^{-} \rightarrow$ $M_{0}^{-} \times M_{1} \times \cdots \times M_{r}^{-} \times M_{0}, Z \mapsto Z^{T}$ is the transposition of the first to the last factor. 
In Section 4.3 this transversality will be achieved by a suitable Hamiltonian isotopy. It ensures that the above transverse intersection cuts out a finite set, which we identify with the generalized intersection points

$$
\begin{aligned}
\mathcal{I}(\underline{L}) & :=\times_{\Delta_{M_{0}}}\left(L_{01} \times{ }_{\Delta_{M_{1}}} L_{12} \cdots \times \times_{\Delta_{M_{r}}} L_{r(r+1)}\right) \\
& =\left\{\underline{x}=\left(x_{0}, \ldots, x_{r}\right) \in M_{0} \times \cdots \times M_{r} \mid\left(x_{0}, x_{1}\right) \in L_{01}, \ldots,\left(x_{r}, x_{0}\right) \in L_{r(r+1)}\right\} .
\end{aligned}
$$

Remark 3.1.3 Consider two cyclic generalized Lagrangian correspondences

$$
\begin{aligned}
\underline{L} & =\left(L_{01}, \ldots, L_{(j-1) j}, L_{j(j+1)}, \ldots, L_{r(r+1)}\right), \\
\underline{L}^{\prime} & =\left(L_{01}, \ldots, L_{(j-1) j} \circ L_{j(j+1)}, \ldots, L_{r(r+1)}\right)
\end{aligned}
$$

such that the composition $L_{(j-1) j} \circ L_{j(j+1)}$ is embedded in the sense of Definition 2.0.4. Then the generalized intersection points

$$
\begin{gathered}
\mathcal{I}(\underline{L})=\left\{\left(\ldots, x_{j-1}, x_{j}, x_{j+1}, \ldots\right) \in \cdots \times M_{j-1} \times M_{j} \times M_{j+1} \cdots \mid\right. \\
\left.\ldots,\left(x_{j-1}, x_{j}\right) \in L_{(j-1) j},\left(x_{j}, x_{j+1}\right) \in L_{j(j+1)}, \ldots\right\} \\
=\left\{\left(\ldots, x_{j-1}, x_{j+1}, \ldots\right) \in \cdots \times M_{j-1} \times M_{j+1} \cdots \mid\right. \\
\left.\ldots,\left(x_{j-1}, x_{j+1}\right) \in L_{(j-1) j} \circ L_{j(j+1)}, \ldots\right\}=\mathcal{I}\left(\underline{L}^{\prime}\right)
\end{gathered}
$$

are canonically identified, since the intermediate point $x_{j} \in M_{j}$ with $\left(x_{j-1}, x_{j}\right) \in$ $L_{(j-1) j}$ and $\left(x_{j}, x_{j+1}\right) \in L_{j(j+1)}$ is uniquely determined by the pair $\left(x_{j-1}, x_{j+1}\right) \in$ $L_{(j-1) j} \circ L_{j(j+1)}$.

An $N$-grading on $\underline{L}$ induces an $N$-fold Maslov covering on

$$
M:=M_{0}^{-} \times M_{1} \times \cdots \times M_{r} \times M_{r}^{-} \times M_{0}
$$

and a grading of

$$
L:=L_{01} \times L_{12} \times \cdots \times L_{r(r+1)} .
$$

In addition, we have a grading on

$$
\Delta^{T}:=\left(\Delta_{M_{0}}^{-} \times \Delta_{M_{1}}^{-} \times \cdots \times \Delta_{M_{r}}^{-}\right)^{T}
$$

from the canonical grading on each factor. In order to define a degree we then identify generalized intersection points $\underline{x}=\left(x_{0}, x_{1}, \ldots, x_{r}\right)$ with the actual intersection points $x=\left(x_{0}, x_{1}, x_{1}, \ldots, x_{r}, x_{r}, x_{0}\right) \in L \cap \Delta^{T}$.

Definition 3.1.4 Let $\underline{L}$ be a graded cyclic generalized Lagrangian correspondence $\underline{L}$ that is transverse to the diagonal (13). Then the degree is

$$
\mathcal{I}(\underline{L}) \rightarrow \mathbb{Z}_{N}, \quad \underline{x} \mapsto|\underline{x}|=d\left(\sigma_{L}^{N}(x), \sigma_{\Delta^{T}}^{N}(x)\right) .
$$


Lemma 3.1.5 Alternatively, the degree is defined as follows:

(a) Pick any tuple of Lagrangian subspaces $\Lambda_{i}^{\prime} \in \operatorname{Lag}\left(T_{x_{i}} M_{i}\right), \Lambda_{i}^{\prime \prime} \in \operatorname{Lag}\left(T_{x_{i}} M_{i}^{-}\right)$, $i=0, \ldots, r$ whose product is transverse to the diagonal, $\Lambda_{i}^{\prime} \times \Lambda_{i}^{\prime \prime} \pitchfork \Delta_{T_{x_{i}}} M_{i}$. Then there exists a path (unique up to homotopy) $\gamma:[0,1] \rightarrow \operatorname{Lag}\left(T_{x} M\right)$ from $\gamma(0)=T_{x} L$ to $\gamma(1)=\Lambda_{0}^{\prime \prime} \times \Lambda_{1}^{\prime} \times \cdots \times \Lambda_{r}^{\prime} \times \Lambda_{r}^{\prime \prime} \times \Lambda_{0}^{\prime}$ that is transverse to the diagonal at all times, $\gamma(t) \pitchfork T_{x} \Delta^{T}$. We lift the grading $\sigma_{L}^{N}(x) \in \operatorname{Lag}^{N}\left(T_{x} M\right)$ along this path and pick preimages under the graded product map (10) to define $\tilde{\Lambda}_{i}^{\prime} \in \operatorname{Lag}^{N}\left(T_{x_{i}} M_{i}\right)$ and $\tilde{\Lambda}_{i}^{\prime \prime} \in \operatorname{Lag}^{N}\left(T_{x_{i}} M_{i}^{-}\right)$. Then

$$
|\underline{x}|=\sum_{i=0}^{r} d\left(\tilde{\Lambda}_{i}^{\prime}, \tilde{\Lambda}_{i}^{\prime \prime-}\right) .
$$

(b) If $\underline{L}$ has even length $r+1 \in 2 \mathbb{N}$ then it defines an $N$-fold Maslov cover on $\widetilde{M}:=M_{0}^{-} \times M_{1} \times M_{2}^{-} \times \cdots \times M_{r}$ and a pair of graded Lagrangian submanifolds,

$$
\begin{aligned}
& L_{(0)}:=L_{01} \times L_{23} \times \cdots \times L_{(r-1) r} \subset \widetilde{M}, \\
& L_{(1)}:=\left(L_{12} \times L_{34} \times \cdots \times L_{r(r+1)}\right)^{T} \subset \widetilde{M}^{-},
\end{aligned}
$$

where we denote by $M_{1}^{-} \times \cdots \times M_{r}^{-} \times M_{0} \rightarrow M_{0} \times M_{1}^{-} \times \cdots \times M_{r}^{-}, Z \mapsto Z^{T}$ the transposition of the last to the first factor. If $\underline{L}$ has odd length $r+1 \in 2 \mathbb{N}+1$ we insert the diagonal $\Delta_{M_{0}} \subset M_{0}^{-} \times M_{0}=M_{r+1}^{-} \times M_{0}$ (with its canonical grading) before defining a pair of graded Lagrangian submanifolds as above. By (13), the Lagrangians intersect transversally $L_{(0)} \pitchfork L_{(1)}^{-}$, and this intersection is canonically identified with $\mathcal{I}(\underline{L})$. Then for $\underline{x} \in \mathcal{I}(\underline{L})$ corresponding to $y \in L_{(0)} \cap L_{(1)}^{-}$we have

$$
|\underline{x}|=|y|=d\left(\sigma_{L_{(0)}}^{N}(y), \sigma_{L_{(1)}}^{N}(y)^{-}\right) .
$$

Proof In (a) we use the fact that the path $\gamma$ has zero Maslov index to rewrite $d\left(\sigma_{L}^{N}(x), \sigma_{\Delta^{T}}^{N}(x)\right)=d\left(\tilde{\Lambda}_{0}^{\prime} \times{ }^{N} \tilde{\Lambda}_{0}^{\prime \prime} \times{ }^{N} \cdots \times{ }^{N} \widetilde{\Lambda}_{r}^{\prime} \times{ }^{N} \widetilde{\Lambda}_{r}^{\prime \prime}, \tilde{\Delta}_{T_{x_{0}} M_{0}} \times{ }^{N} \cdots \times{ }^{N} \tilde{\Delta}_{T_{x_{r}} M_{r}}^{-}\right)$,

where we moreover transposed the factors. Now by Lemma 3.0.12 the right-hand side can be written as the sum over $d\left(\tilde{\Lambda}_{i}^{\prime} \times{ }^{N} \tilde{\Lambda}_{i}^{\prime \prime}, \tilde{\Delta}_{T_{x_{i}} M_{i}}^{-}\right)=d\left(\tilde{\Lambda}_{i}^{\prime}, \tilde{\Lambda}_{i}^{\prime \prime-}\right)$.

In (b) note that a reordering of the factors identifies the pair of graded Lagrangians $\left(L_{(0)} \times L_{(1)}, \Delta_{\widetilde{M}}^{-}\right)$with $\left(L, \Delta^{T}\right)$ for $r$ odd. So Lemma 3.0.12 implies $d\left(\sigma_{L}^{N}(x), \sigma_{\Delta^{T}}^{N}(x)\right)=d\left(\sigma_{L_{(0)}}^{N}(y) \times{ }^{N} \sigma_{L_{(1)}}^{N}(y), \tilde{\Delta}_{T_{(y, y)}}^{-} \widetilde{M}^{)}=d\left(\sigma_{L_{(0)}}^{N}(y), \sigma_{L_{(1)}}^{N}(y)^{-}\right)\right.$. 
For $r$ even the same argument proves

$$
\begin{aligned}
& d\left(\sigma_{L_{(0)}}^{N}(y), \sigma_{L_{(1)}}^{N}(y)^{-}\right) \\
& \quad=d\left(\sigma_{L}^{N}(x) \times{ }^{N} \widetilde{\Delta}_{T_{x_{0}} M_{0}},\left(\widetilde{\Delta}_{T_{x_{0}} M_{0}}^{-} \times \cdots \times \widetilde{\Delta}_{T_{x_{r}} M_{r}}^{-} \times \widetilde{\Delta}_{T_{x_{0}} M_{0}}^{-}\right)^{T}\right),
\end{aligned}
$$

which equals to $d\left(\sigma_{L}^{N}(x), \sigma_{\Delta^{T}}^{N}(x)\right)$ by Lemma 3.1.6 (b) below.

The following Lemma describes the effect of inserting a diagonal on the grading of generalized Lagrangian correspondences. Part (a) addresses noncyclic correspondences, whereas (b) applies to cyclic correspondences with

$$
\begin{aligned}
\Lambda & =T_{\left(x_{0}, x_{1}, \ldots, x_{r}, x_{0}\right)}\left(L_{01} \times L_{12} \times \cdots \times L_{r(r+1)}\right), \\
K & =T_{\left(x_{0}, x_{0}, x_{1}, \ldots, x_{r}\right)}\left(\Delta_{M_{0}}^{-} \times \Delta_{M_{1}}^{-} \times \cdots \times \Delta_{M_{r}}^{-}\right), \\
V_{0} & =T_{x_{0}} M_{0}, \\
V_{1} & =T_{\left(x_{1}, \ldots, x_{r}\right)}\left(M_{1} \times M_{1}^{-} \times \cdots \times M_{r} \times M_{r}^{-}\right) .
\end{aligned}
$$

Lemma 3.1.6 Let $V_{0}, V_{1}, V_{2}$ be symplectic vector spaces.

(a) Let $\tilde{\Lambda}_{0} \subset \operatorname{Lag}^{N}\left(V_{0}\right), \tilde{\Lambda}_{01} \subset \operatorname{Lag}^{N}\left(V_{0}^{-} \times V_{1}\right), \tilde{\Lambda}_{12} \subset \operatorname{Lag}^{N}\left(V_{1}^{-} \times V_{2}\right)$, and $\widetilde{\Lambda}_{2} \subset \operatorname{Lag}^{N}\left(V_{2}^{-}\right)$be graded Lagrangian subspaces. If the underlying Lagrangian subspaces are transverse then

$$
d\left(\tilde{\Lambda}_{0} \times{ }^{N} \tilde{\Lambda}_{12}, \tilde{\Lambda}_{01}^{-} \times^{N} \tilde{\Lambda}_{2}^{-}\right)=d\left(\tilde{\Lambda}_{0} \times^{N} \tilde{\Delta}_{1} \times^{N} \tilde{\Lambda}_{2}, \tilde{\Lambda}_{01}^{-} \times^{N} \tilde{\Lambda}_{12}^{-}\right) .
$$

(b) Let $\tilde{\Lambda} \subset \operatorname{Lag}^{N}\left(V_{0}^{-} \times V_{1} \times V_{0}\right)$ and $\tilde{K} \subset \operatorname{Lag}^{N}\left(V_{0} \times V_{0}^{-} \times V_{1}\right)$ be graded Lagrangian subspaces. If the underlying Lagrangian subspaces are transverse then

$$
d\left(\tilde{\Lambda} \times{ }^{N} \widetilde{\Delta}_{0},\left(\tilde{K} \times^{N} \tilde{\Delta}_{0}^{-}\right)^{T}\right)=d\left(\tilde{\Lambda}, \tilde{K}^{T}\right),
$$

with the transposition $V_{0} \times W \rightarrow W \times V_{0}, Z \mapsto Z^{T}$.

Proof To prove (a) pick a path $\gamma_{0112}:[0,1] \rightarrow \operatorname{Lag}\left(V_{0} \times V_{1}^{-} \times V_{1} \times V_{2}^{-}\right)$from $\gamma_{0112}(0)=\Lambda_{01}^{-} \times \Lambda_{12}^{-}$to a split Lagrangian subspace $\gamma_{0112}(1)=\Lambda_{0}^{\prime} \times \Lambda_{1}^{\prime} \times \Lambda_{1}^{\prime \prime} \times \Lambda_{2}^{\prime}$ that is transverse to $\Lambda_{0} \times \Delta_{1} \times \Lambda_{2}$ at all times and hence has Maslov index $I\left(\gamma_{0112}, \Lambda_{0} \times\right.$ $\left.\Delta_{1} \times \Lambda_{2}\right)=0$. We can homotope this path with fixed endpoints to $\gamma_{0112}=\gamma_{01} \times$ $\gamma_{12}:[0,1] \rightarrow \operatorname{Lag}\left(V_{0} \times V_{1}^{-}\right) \times \operatorname{Lag}\left(V_{1} \times V_{2}^{-}\right)$that may intersect $\Lambda_{0} \times \Delta_{1} \times \Lambda_{2}$ but still has vanishing Maslov index. We lift the grading along the paths $\gamma_{01}$ and $\gamma_{12}$ and pick preimages under the graded product map (10) to obtain gradings $\widetilde{\Lambda}_{0}^{\prime} \in \operatorname{Lag}^{N}\left(V_{0}\right)$, $\widetilde{\Lambda}_{1}^{\prime} \in \operatorname{Lag}^{N}\left(V_{1}^{-}\right), \tilde{\Lambda}_{1}^{\prime \prime} \in \operatorname{Lag}^{N}\left(V_{1}\right), \tilde{\Lambda}_{2}^{\prime} \in \operatorname{Lag}^{N}\left(V_{2}^{-}\right)$. With these we calculate, 
using Lemma 3.0.12,

$$
\begin{aligned}
d\left(\tilde{\Lambda}_{0} \times{ }^{N} \tilde{\Lambda}_{12}, \tilde{\Lambda}_{01}^{-} \times{ }^{N} \tilde{\Lambda}_{2}^{-}\right) & =d\left(\tilde{\Lambda}_{0} \times{ }^{N} \tilde{\Lambda}_{1}^{\prime \prime} \times{ }^{N} \tilde{\Lambda}_{2}^{\prime-}, \tilde{\Lambda}_{0}^{\prime} \times{ }^{N} \tilde{\Lambda}_{1}^{\prime} \times{ }^{N} \tilde{\Lambda}_{2}^{-}\right) \\
& =d\left(\tilde{\Lambda}_{0}, \tilde{\Lambda}_{0}^{\prime}\right)+d\left(\tilde{\Lambda}_{1}^{\prime \prime-}, \tilde{\Lambda}_{1}^{\prime}\right)+d\left(\tilde{\Lambda}_{2}^{\prime-}, \tilde{\Lambda}_{2}^{-}\right) \\
& =d\left(\tilde{\Lambda}_{0}, \tilde{\Lambda}_{0}^{\prime}\right)+d\left(\tilde{\Delta}_{1}, \tilde{\Lambda}_{1}^{\prime} \times{ }^{N} \tilde{\Lambda}_{1}^{\prime \prime}\right)+d\left(\tilde{\Lambda}_{2}, \tilde{\Lambda}_{2}^{\prime}\right) \\
& =d\left(\tilde{\Lambda}_{0} \times{ }^{N} \tilde{\Delta}_{1} \times{ }^{N} \tilde{\Lambda}_{2}, \tilde{\Lambda}_{0}^{\prime} \times{ }^{N} \tilde{\Lambda}_{1}^{\prime} \times{ }^{N} \tilde{\Lambda}_{1}^{\prime \prime} \times{ }^{N} \tilde{\Lambda}_{2}^{\prime}\right) \\
& =d\left(\tilde{\Lambda}_{0} \times{ }^{N} \tilde{\Delta}_{1} \times{ }^{N} \tilde{\Lambda}_{2}, \tilde{\Lambda}_{01}^{-} \times{ }^{N} \tilde{\Lambda}_{12}^{-}\right) .
\end{aligned}
$$

The first and last degree identity are due to the vanishing of the Maslov index

$$
0=I\left(\Lambda_{0} \times \Delta_{1} \times \Lambda_{2}, \gamma_{01} \times \gamma_{12}\right)=I\left(\Lambda_{0} \times \gamma_{12}^{-}, \gamma_{01} \times \Lambda_{2}^{-}\right)=0
$$

The identity of these Maslov indices follows from identifying the intersections $K(s):=$ $\left(\Lambda_{0} \times \gamma_{12}^{-}(s)\right) \cap\left(\gamma_{01}(s) \times \Lambda_{2}^{-}\right) \cong\left(\Lambda_{0} \times \Delta_{1} \times \Lambda_{2}\right) \cap\left(\gamma_{01} \times \gamma_{12}\right)$ and the crossing form $\Gamma(s), \hat{\Gamma}(s): K(s) \rightarrow \mathbb{R}$ given by (12) at regular crossings $s \in[0,1]$. Fix Lagrangian complements $\gamma_{01}(s)^{c} \subset V_{0} \times V_{1}^{-}$and $\gamma_{12}(s)^{c} \subset V_{1} \times V_{2}^{-}$, then for $v=\left(v_{0}, v_{1}, v_{2}\right) \in K(s)$ we can pick $\left(w_{1}, w_{2}\right)(t) \in \gamma_{12}(s)^{c}$ such that $v+\left(0, w_{1}, w_{2}\right)(t) \in \Lambda_{0} \times \gamma_{12}(s+t)$ and $\left(w_{0}^{\prime}, w_{1}^{\prime}\right)(t) \in \gamma_{01}(s)^{c}$ such that $v+\left(w_{0}^{\prime}, w_{1}^{\prime}, 0\right)(t) \in \gamma_{01}(s+t) \times \Lambda_{2}$. For the corresponding vector $\hat{v}=\left(v_{0}, v_{1}, v_{1}, v_{2}\right) \in\left(\Lambda_{0} \times \Delta_{1} \times \Lambda_{2}^{-}\right) \cap\left(\gamma_{01}^{-} \times \gamma_{12}^{-}\right)$we have $\widehat{v}+(0,0,0,0) \in\left(\Lambda_{0} \times \Delta_{1} \times \Lambda_{2}\right)$ and $\widehat{v}+\left(w_{0}^{\prime}, w_{1}^{\prime}, w_{1}, w_{2}\right)(t) \in\left(\gamma_{01} \times \gamma_{12}\right)(s+t)$. With this we identify the crossing forms

$$
\begin{aligned}
\widehat{\Gamma}(s) \widehat{v} & =\left.\frac{d}{d t}\right|_{t=0}\left(\omega_{0} \oplus-\omega_{1} \oplus \omega_{1} \oplus-\omega_{2}\right)\left(\widehat{v},(0,0,0,0)-\left(w_{0}^{\prime}, w_{1}^{\prime}, w_{1}, w_{2}\right)(t)\right) \\
& =\left.\frac{d}{d t}\right|_{t=0}\left(-\omega_{0}\left(v_{0}, w_{0}^{\prime}\right)-\omega_{1}\left(v_{1}, w_{1}-w_{1}^{\prime}\right)+\omega_{2}\left(v_{2}, w_{2}\right)\right) \\
& =\left.\frac{d}{d t}\right|_{t=0}\left(\omega_{0} \oplus-\omega_{1} \oplus \omega_{2}\right)\left(v,\left(0, w_{1}, w_{2}\right)(t)-\left(w_{0}^{\prime}, w_{1}^{\prime}, 0\right)(t)\right)=\Gamma(s) v .
\end{aligned}
$$

This proves (a). To prove (b) we pick a path $\gamma:[0,1] \rightarrow \operatorname{Lag}\left(V_{0}^{-} \times V_{1} \times V_{0}\right)$ from $\gamma(0)=$ $\Lambda$ to a split Lagrangian subspace $\gamma(1)=\Lambda_{0}^{-} \times \Lambda_{1} \times \Lambda_{0}^{\prime} \in \operatorname{Lag}\left(V_{0}^{-}\right) \times \operatorname{Lag}\left(V_{1}\right) \times \operatorname{Lag}\left(V_{0}\right)$ that is transverse to $K^{T}$ at all times and hence has Maslov index

$$
0=I\left(\gamma, K^{T}\right)=I\left(\gamma \times \Delta_{0},\left(K \times \Delta_{0}^{-}\right)^{T}\right)
$$

Here the equality of Maslov follows directly from the identification of the trivial intersections $\left(\gamma \times \Delta_{0}\right) \cap\left(K \times \Delta_{0}^{-}\right)^{T} \cong \gamma \cap K^{T}=\{0\}$. Now we can lift the grading along $\gamma$ to obtain gradings $\tilde{\Lambda}_{0} \in \operatorname{Lag}^{N}\left(V_{0}\right), \tilde{\Lambda}_{1} \in \operatorname{Lag}^{N}\left(V_{1}\right), \tilde{\Lambda}_{0}^{\prime} \in \operatorname{Lag}^{N}\left(V_{0}\right)$. With these we calculate, using part (a) and the fact that gradings are invariant under 
simultaneous transposition of both factors,

$$
\begin{aligned}
d\left(\tilde{\Lambda} \times{ }^{N} \tilde{\Delta}_{0},\left(\tilde{K} \times{ }^{N} \tilde{\Delta}_{0}^{-}\right)^{T}\right) & =d\left(\tilde{\Lambda}_{0}^{-} \times{ }^{N} \tilde{\Lambda}_{1} \times{ }^{N} \tilde{\Lambda}_{0}^{\prime} \times{ }^{N} \tilde{\Delta}_{0},\left(\tilde{K} \times{ }^{N} \tilde{\Delta}_{0}^{-}\right)^{T}\right) \\
& =d\left(\tilde{\Lambda}_{0}^{\prime} \times{ }^{N} \tilde{\Delta}_{0} \times{ }^{N} \tilde{\Lambda}_{0}^{-} \times{ }^{N} \tilde{\Lambda}_{1}, \tilde{\Delta}_{0}^{-} \times{ }^{N} \tilde{K}\right) \\
& =d\left(\tilde{\Lambda}_{0}^{\prime} \times{ }^{N} \tilde{K}^{-}, \tilde{\Delta}_{0}^{-} \times{ }^{N}\left(\tilde{\Lambda}_{0}^{-} \times{ }^{N} \tilde{\Lambda}_{1}\right)^{-}\right) \\
& =d\left(\tilde{\Delta}_{0} \times{ }^{N}\left(\tilde{\Lambda}_{0}^{-} \times{ }^{N} \tilde{\Lambda}_{1}\right), \tilde{\Lambda}_{0}^{\prime-} \times{ }^{N} \tilde{K}\right) \\
& =d\left(\tilde{K}^{-}, \tilde{\Lambda}_{0}^{\prime-} \times{ }^{N}\left(\tilde{\Lambda}_{0}^{-} \times{ }^{N} \tilde{\Lambda}_{1}\right)^{-}\right) \\
& =d\left(\tilde{\Lambda}_{0}^{\prime} \times{ }^{N} \tilde{\Lambda}_{0}^{-} \times{ }^{N} \tilde{\Lambda}_{1}, \tilde{K}\right) \\
& =d\left(\tilde{\Lambda}_{0}^{-} \times{ }^{N} \tilde{\Lambda}_{1} \times{ }^{N} \tilde{\Lambda}_{0}^{\prime}, \tilde{K}^{T}\right)=d\left(\tilde{\Lambda}, \tilde{K}^{T}\right)
\end{aligned}
$$

In the rest of this section we investigate the effect of geometric composition on the grading of Lagrangian correspondences. This requires a generalization of Viterbo's index calculations [36].

First, we lift the composition map to Maslov covers. Let $M_{0}, M_{1}, M_{2}$ be symplectic manifolds equipped with $N$-fold Maslov coverings $\operatorname{Lag}^{N}\left(M_{j}\right), j=0,1,2$. We equip the products $M_{i}^{-} \times M_{j}$ and $M_{0}^{-} \times M_{1} \times M_{1}^{-} \times M_{2}$ with the induced Maslov coverings $\operatorname{Lag}^{N}\left(M_{i}^{-} \times M_{j}\right)$ resp. $\operatorname{Lag}^{N}\left(M_{0}^{-} \times M_{1} \times M_{1}^{-} \times M_{2}\right)$. We denote by

$$
\left.\mathcal{T}\left(M_{1}\right) \subset \operatorname{Lag}\left(M_{0}^{-} \times M_{1} \times M_{1}^{-} \times M_{2}\right)\right|_{M_{0} \times \Delta_{M_{1}} \times M_{2}}
$$

the subbundle whose fibre over $\left(m_{0}, m_{1}, m_{1}, m_{2}\right)$ consists of the Lagrangian subspaces

$$
\Lambda_{0112} \subset T_{\left(m_{0}, m_{1}, m_{1}, m_{2}\right)}\left(M_{0}^{-} \times M_{1} \times M_{1}^{-} \times M_{2}\right)
$$

that are transverse to the diagonal

$$
\Delta_{0112}:=T_{m_{0}} M_{0} \times \Delta_{T_{m_{1}} M_{1}} \times T_{m_{2}} M_{2} .
$$

The linear composition of Lagrangian subspaces extends a smooth map

$$
\circ: \mathcal{T}\left(M_{1}\right) \rightarrow \operatorname{Lag}\left(M_{0}^{-} \times M_{2}\right), \quad \Lambda_{0112} \mapsto \pi_{M_{0} \times M_{2}}\left(\Lambda_{0112} \cap \Delta_{0112}\right) .
$$

The preimage of $\mathcal{T}\left(M_{1}\right)$ in the Maslov cover will be denoted by

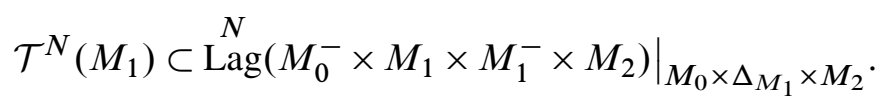

Finally, recall that we have a canonical grading of the diagonal $\widetilde{\Delta}_{M_{1}} \in \operatorname{Lag}^{N}\left(M_{1}^{-} \times M_{1}\right)$ and its dual $\widetilde{\Delta}_{M_{1}}^{-} \in \operatorname{Lag}^{N}\left(M_{1} \times M_{1}^{-}\right)$, and let us denote another exchange of factors by

$$
\stackrel{N}{\operatorname{Lag}}\left(M_{0}^{-} \times M_{2} \times M_{1} \times M_{1}^{-}\right) \rightarrow \stackrel{N}{\operatorname{Lag}}\left(M_{0}^{-} \times M_{1} \times M_{1}^{-} \times M_{2}\right), \quad \tilde{\Lambda} \mapsto \tilde{\Lambda}^{T} .
$$


Lemma 3.1.7 The linear composition $\circ: \mathcal{T}\left(M_{1}\right) \rightarrow \operatorname{Lag}\left(M_{0}^{-} \times M_{2}\right)$ lifts to a unique smooth map $\circ^{N}: \mathcal{T}^{N}\left(M_{1}\right) \rightarrow \operatorname{Lag}^{N}\left(M_{0}^{-} \times M_{2}\right)$ with the property that

$$
{ }^{N}\left(\left(\tilde{\Lambda}_{02} \times{ }^{N} \tilde{\Lambda}_{11}\right)^{T}\right)=d\left(\tilde{\Lambda}_{11}, \tilde{\Delta}_{M_{1}}^{-}\right) \cdot \tilde{\Lambda}_{02}
$$

for all graded Lagrangians $\widetilde{\Lambda}_{02} \in \operatorname{Lag}^{N}\left(M_{0}^{-} \times M_{2}\right)$ and $\tilde{\Lambda}_{11} \in \operatorname{Lag}^{N}\left(M_{1} \times M_{1}^{-}\right)$, such that the underlying Lagrangian $\Lambda_{11} \in \operatorname{Lag}\left(M_{1} \times M_{1}^{-}\right)$is transverse to the diagonal.

Proof We denote by $\operatorname{Lag}\left(\mathbb{R}^{2 n}\right)$ the Lagrangian Grassmannian in $\mathbb{R}^{2 n}$, write $\operatorname{dim} M_{i}=$ $2 n_{i}$, and abbreviate $\mathbb{R}_{0112}:=\mathbb{R}^{2 n_{0},-} \times \mathbb{R}^{2 n_{1}} \times \mathbb{R}^{2 n_{1},-} \times \mathbb{R}^{2 n_{2}}$. Let $\mathcal{T} \subset \operatorname{Lag}\left(\mathbb{R}_{0112}\right)$ be the subset of Lagrangian subspaces meeting the diagonal $\mathbb{R}^{2 n_{0}} \times \Delta_{\mathbb{R}^{2 n_{1}}} \times \mathbb{R}^{2 n_{2}}$ transversally. The linear composition map

$\operatorname{Lag}\left(\mathbb{R}_{0112}\right) \supset \mathcal{T} \rightarrow \operatorname{Lag}\left(\mathbb{R}^{2 n_{0},-} \times \mathbb{R}^{2 n_{2}}\right), \Lambda \mapsto \pi_{\mathbb{R}^{2 n_{0}} \times \mathbb{R}^{2 n_{2}}}\left(\Lambda \cap\left(\mathbb{R}^{2 n_{0}} \times \Delta_{\mathbb{R}^{2 n_{1}}} \times \mathbb{R}^{2 n_{2}}\right)\right)$ is equivariant under $\operatorname{Sp}\left(2 n_{0}\right) \times \operatorname{Sp}\left(2 n_{1}\right) \times \operatorname{Sp}\left(2 n_{2}\right)$, and it lifts to a unique map equivariant under $\operatorname{Sp}^{N}\left(2 n_{0}\right) \times \operatorname{Sp}^{N}\left(2 n_{1}\right) \times \operatorname{Sp}^{N}\left(2 n_{2}\right)$,

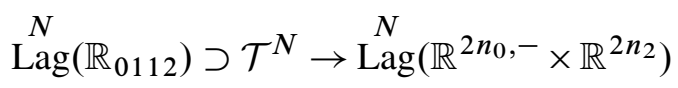

with the property (14). On the other hand, the restriction of the product bundle $\operatorname{Fr}\left(M_{0}\right) \times \operatorname{Fr}\left(M_{1}\right) \times \operatorname{Fr}\left(M_{1}\right) \times \operatorname{Fr}\left(M_{2}\right)$ to $M_{0} \times \Delta_{M_{1}} \times M_{2}$ admits a reduction of the structure group to $\operatorname{Sp}\left(2 n_{0}\right) \times \operatorname{Sp}\left(2 n_{1}\right) \times \operatorname{Sp}\left(2 n_{2}\right)$, and similarly the restriction

$$
\underset{0112}{\mathrm{Fr}}:=\left.\left(\stackrel{N}{\operatorname{Fr}}\left(M_{0}\right) \times \stackrel{N}{\operatorname{Fr}}\left(M_{1}\right) \times \stackrel{N}{\operatorname{Fr}}\left(M_{1}\right) \times \stackrel{N}{\operatorname{Fr}}\left(M_{2}\right)\right)\right|_{M_{0} \times \Delta_{M_{1}} \times M_{2}}
$$

admits a reduction of the structure group to $\operatorname{Sp}^{N}\left(2 n_{0}\right) \times \operatorname{Sp}^{N}\left(2 n_{1}\right) \times \operatorname{Sp}^{N}\left(2 n_{2}\right)$. This group acts on $\operatorname{Lag}^{N}\left(\mathbb{R}_{0112}\right)$ by the diagonal action of $\operatorname{Sp}^{N}\left(2 n_{1}\right)$ on $\mathbb{R}^{2 n_{1}} \times \mathbb{R}^{2 n_{1},-}$. Finally, we use the associated fiber bundle construction to identify

$$
\begin{aligned}
& \left.\stackrel{N}{\operatorname{Lag}\left(M_{0}^{-} \times M_{1} \times M_{1}^{-} \times M_{2}\right)}\right|_{M_{0} \times \Delta_{M_{1}} \times M_{2}} \\
& \cong \underset{0112}{N} \times{ }_{\operatorname{Sp}^{N}}^{N}\left(2 n_{0}\right) \times \operatorname{Sp}^{N}\left(2 n_{1}\right) \times \operatorname{Sp}^{N}\left(2 n_{1}\right) \times \operatorname{Sp}^{N}\left(2 n_{2}\right) \stackrel{N}{\operatorname{Lag}\left(\mathbb{R}_{0112}\right)} \\
& \cong\left(\stackrel{N}{\mathrm{Fr}}\left(M_{0}\right) \times \stackrel{N}{\mathrm{Fr}}\left(M_{1}\right) \times \stackrel{N}{\mathrm{Fr}}\left(M_{2}\right)\right) \times \times_{\mathrm{Sp}^{N}\left(2 n_{0}\right) \times \mathrm{Sp}^{N}\left(2 n_{1}\right) \times \mathrm{Sp}^{N}\left(2 n_{2}\right)} \stackrel{N}{\operatorname{Lag}\left(\mathbb{R}_{0112}\right)}, \\
& \stackrel{N}{\operatorname{Lag}}\left(M_{0}^{-} \times M_{2}\right) \\
& =\left(\stackrel{N}{\operatorname{Fr}}\left(M_{0}^{-}\right) \times \stackrel{N}{\operatorname{Fr}}\left(M_{2}\right)\right) \times_{\mathrm{Sp}^{N}\left(2 n_{0}\right) \times \mathrm{Sp}^{N}\left(2 n_{2}\right)} \stackrel{N a g}{\operatorname{Lag}\left(\mathbb{R}^{2 n_{0},-} \times \mathbb{R}^{2 n_{2}}\right) .}
\end{aligned}
$$

Then the forgetful map on the first factor and the equivariant map (15) on the second factor define the unique lift $\circ^{N}$. 
Now consider two graded Lagrangian correspondences $L_{01} \subset M_{0}^{-} \times M_{1}$ and $L_{12} \subset$ $M_{1}^{-} \times M_{2}$ and suppose that the composition $L_{01} \circ L_{12}=: L_{02} \subset M_{0}^{-} \times M_{2}$ is smooth and embedded. The canonical section $\sigma_{L_{02}}: L_{02} \rightarrow \operatorname{Lag}\left(M_{0}^{-} \times M_{2}\right)$ is given by the linear composition $\circ$ applied to $\left.\left(\sigma_{L_{01}} \times \sigma_{L_{12}}\right)\right|_{L_{01} \times \Delta_{M_{1}}} L_{12}$. The gradings $\sigma_{L_{01}}^{N}, \sigma_{L_{12}}^{N}$ induce a grading on $L_{02}$,

$$
\sigma_{L_{02}}^{N}:=\left.\circ^{N}\left(\sigma_{L_{01}}^{N} \times{ }^{N} \sigma_{L_{12}}^{N}\right)\right|_{L_{01} \times \Delta_{M_{1}} L_{12}},
$$

where the map $\times^{N}$ is defined in (10) and we identify $L_{02} \cong L_{01} \times_{\Delta_{M_{1}}} L_{12}$.

Proposition 3.1.8 Let $L_{0} \subset M_{0}, L_{01} \subset M_{0}^{-} \times M_{1}, L_{12} \subset M_{1}^{-} \times M_{2}$, and $L_{2} \subset M_{2}^{-}$ be graded Lagrangians such that the composition $L_{01} \circ L_{12}=: L_{02}$ is embedded. Then, with respect to the induced grading on $L_{02}$, the degree map $\mathcal{I}\left(L_{0} \times L_{2}, L_{02}\right) \rightarrow \mathbb{Z}_{N}$ is the pullback of the degree map $\mathcal{I}\left(L_{0} \times L_{12}, L_{01} \times L_{2}\right) \rightarrow \mathbb{Z}_{N}$ under the canonical identification $^{3}$ of intersection points.

Proof Suppose for simplicity that Hamiltonian perturbations have been applied to the Lagrangians $L_{0}, L_{2}$ such that $\mathcal{I}\left(L_{0} \times L_{2}, L_{02}\right)$ (and hence also $\mathcal{I}\left(L_{0} \times L_{12}, L_{01} \times L_{2}\right)$ ) is the intersection of transverse Lagrangians. We need to consider $\left(m_{0}, m_{1}, m_{2}\right) \in$ $\left(L_{0} \times L_{12}\right) \cap\left(L_{01} \times L_{2}\right)$, which corresponds to $\left(m_{0}, m_{2}\right) \in\left(L_{0} \times L_{2}\right) \cap L_{02}$. We abbreviate the tangent spaces of the Lagrangians by $\Lambda_{j}=T_{m_{j}} L_{j}, \Lambda_{i j}=T_{\left(m_{i}, m_{j}\right)} L_{i j}$, and $\Delta_{1}=\Delta_{T_{m_{1}} M_{1}}$ and their graded lifts by $\tilde{\Lambda}_{j}=\sigma_{L_{j}}^{N}\left(m_{j}\right), \widetilde{\Lambda}_{i j}=\sigma_{L_{i j}}^{N}\left(m_{i}, m_{j}\right)$, and $\widetilde{\Delta}_{1}=\widetilde{\Delta}_{T_{m_{1}} M_{1}}$. We claim that

$$
\begin{aligned}
d\left(\tilde{\Lambda}_{0} \times{ }^{N} \tilde{\Lambda}_{12}, \tilde{\Lambda}_{01}^{-} \times{ }^{N} \tilde{\Lambda}_{2}^{-}\right) & =d\left(\tilde{\Lambda}_{0} \times{ }^{N} \tilde{\Delta}_{1} \times{ }^{N} \tilde{\Lambda}_{2}, \tilde{\Lambda}_{01}^{-} \times{ }^{N} \tilde{\Lambda}_{12}^{-}\right) \\
& =d\left(\tilde{\Lambda}_{0} \times{ }^{N} \tilde{\Lambda}_{2}, \tilde{\Lambda}_{01}^{-} \circ^{N} \tilde{\Lambda}_{12}^{-}\right) .
\end{aligned}
$$

The first identity is Lemma 3.1.6. To prove (17) we begin by noting the transverse intersection $\Lambda_{02} \pitchfork \Lambda_{0} \times \Lambda_{2}$. We denote $\widetilde{\Lambda}_{02}:=\widetilde{\Lambda}_{01} \circ^{N} \widetilde{\Lambda}_{12}$ (hence $\widetilde{\Lambda}_{02}^{-}=\tilde{\Lambda}_{01}^{-} \circ^{N} \tilde{\Lambda}_{12}^{-}$) and pick a path $\tilde{\gamma}_{02}:[0,1] \rightarrow \operatorname{Lag}^{N}\left(T_{m_{0}} M_{0}^{-} \times T_{m_{2}} M_{2}\right)$ from $\tilde{\gamma}_{02}(0)=\tilde{\Lambda}_{0} \times{ }^{N} \tilde{\Lambda}_{2}$ to $\tilde{\gamma}_{02}(1)=\tilde{\Lambda}_{02}^{-}$whose crossing form with $\Lambda_{0} \times \Lambda_{2}$ at $s=0$ is positive definite and hence by Remark 3.0.10

$$
d\left(\tilde{\Lambda}_{0} \times^{N} \tilde{\Lambda_{2}}, \tilde{\Lambda}_{02}^{-}\right)=-I^{\prime}\left(\gamma_{02}, \Lambda_{0} \times \Lambda_{2}\right) .
$$

Here $I^{\prime}$ denotes the Maslov index of a pair of paths not counting crossings at the endpoints. Next, fix a complement $L_{11} \in \operatorname{Lag}\left(T_{\left(m_{1}, m_{1}\right)} M_{1} \times M_{1}^{-}\right)$of the diagonal. Then both $\left(\Lambda_{02} \times{ }^{N} L_{11}\right)^{T}$ and $\Lambda_{01} \times \Lambda_{12}$ are transverse to $T_{m_{0}} M_{0} \times \Delta_{1} \times T_{m_{2}} M_{2}$

\footnotetext{
${ }^{3}$ Here it suffices to allow for Hamiltonian perturbation on $M_{0}$ and $M_{2}$, ie replacing $L_{0}, L_{2}$ with $L_{0}^{\prime}:=\phi_{1}^{H_{0}}\left(L_{0}\right), L_{2}^{\prime}:=\left(\phi_{1}^{H_{2}}\right)^{-1}\left(L_{2}\right)$. Then for every $\left(m_{0}, m_{2}\right) \in\left(L_{0}^{\prime} \times L_{2}^{\prime}\right) \cap L_{02}$ there is a unique $m_{1} \in M_{1}$ such that $\left(m_{0}, m_{1}\right) \in L_{01},\left(m_{1}, m_{2}\right) \in L_{12}$, hence $\left(m_{0}, m_{1}, m_{2}\right) \in\left(L_{0}^{\prime} \times L_{12}\right) \cap\left(L_{01} \times L_{2}^{\prime}\right)$.
} 
and their composition is $\Lambda_{02}$. By Lemma 3.1.9 below we then find a path $\gamma_{0112}$ and lift it to $\tilde{\gamma}_{0112}:[0,1] \rightarrow \operatorname{Lag}^{N}\left(T_{\left(m_{0}, m_{1}, m_{1}, m_{2}\right)} M_{0}^{-} \times M_{1} \times M_{1}^{-} \times M_{2}\right)$ from $\tilde{\gamma}_{0112}(0)=$ $\left[\widetilde{\Lambda}_{02} \times{ }^{N} \widetilde{L}_{11}\right]^{T}$ to $\tilde{\gamma}_{0112}(1)=\widetilde{\Lambda}_{01} \times{ }^{N} \widetilde{\Lambda}_{12}$ whose composition $\circ\left(\gamma_{0112}\right)=\Lambda_{02}$ is constant and that has no crossings with $\Lambda_{0} \times \Delta_{1} \times \Lambda_{2}$ (by the transversality $\left.\gamma_{0112} \cap\left(\Lambda_{0} \times \Delta_{1} \times \Lambda_{2}\right)=\Lambda_{02} \cap\left(\Lambda_{0} \times \Lambda_{2}\right)=\{0\}\right)$. Here the grading of $\widetilde{L}_{11}$ is determined by continuation along this path. Since the composition $\circ\left(\gamma_{0112}\right)$ is constant this continuation yields

$$
\tilde{\Lambda}_{02}=\circ^{N}\left(\tilde{\gamma}_{0112}\right)=\circ^{N}\left(\left(\tilde{\Lambda}_{02} \times{ }^{N} \tilde{L}_{11}\right)^{T}\right)=d\left(\tilde{L}_{11}, \tilde{\Delta}_{1}^{-}\right) \cdot \tilde{\Lambda}_{02} \text {. }
$$

Here we also used (14), and we deduce that $d\left(\widetilde{L}_{11}, \widetilde{\Delta}_{1}^{-}\right)=0 \bmod N$. Furthermore, we fix a path $\tilde{\gamma}_{11}:[0,1] \rightarrow \operatorname{Lag}^{N}\left(T_{\left(m_{1}, m_{1}\right)} M_{1}^{-} \times M_{1}\right)$ from $\tilde{\gamma}_{11}(0)=\widetilde{\Delta}_{1}$ to $\tilde{\gamma}_{11}(1)=\widetilde{L}_{11}^{-}$ whose crossing form with $\Delta_{1}$ at $s=0$ is positive definite, and thus

$$
-I^{\prime}\left(\gamma_{11}, \Delta_{1}\right)=d\left(\tilde{\Delta}_{1}, \tilde{L}_{11}^{-}\right)=d\left(\tilde{L}_{11}, \tilde{\Delta}_{1}^{-}\right)=0 \quad \bmod N
$$

Now the concatenated path $\left(\tilde{\gamma}_{02} \times \tilde{\gamma}_{11}\right)^{T} \# \tilde{\gamma}_{0112}^{-}$connects $\tilde{\Lambda}_{0} \times{ }^{N} \tilde{\Delta}_{1} \times{ }^{N} \tilde{\Lambda}_{2}$ to $\widetilde{\Lambda}_{01}^{-} \times{ }^{N} \widetilde{\Lambda}_{12}^{-}$with positive definite crossing form at $s=0$, and (17) can be verified:

$$
\begin{aligned}
d\left(\tilde{\Lambda}_{0} \times{ }^{N} \tilde{\Delta}_{1}\right. & \left.\times N \tilde{\Lambda}_{2}, \tilde{\Lambda}_{01}^{-} \times{ }^{N} \tilde{\Lambda}_{12}^{-}\right) \\
& =-I^{\prime}\left(\left(\gamma_{02} \times \gamma_{11}\right)^{T} \# \gamma_{0112}^{-}, \Lambda_{0} \times \Delta_{1} \times \Lambda_{2}\right) \\
& =-I^{\prime}\left(\gamma_{02}, \Lambda_{0} \times \Lambda_{2}\right)-I^{\prime}\left(\gamma_{11}, \Delta_{1}\right)-I^{\prime}\left(\gamma_{0112}^{-}, \Lambda_{0} \times \Delta_{1} \times \Lambda_{2}\right) \\
& =-I^{\prime}\left(\gamma_{02}, \Lambda_{0} \times \Lambda_{2}\right)=d\left(\tilde{\Lambda}_{0} \times{ }^{N} \tilde{\Lambda}_{2}, \tilde{\Lambda}_{02}^{-}\right) .
\end{aligned}
$$

Lemma 3.1.9 Let $V_{0}, V_{1}, V_{2}$ be symplectic vector spaces, $\Lambda_{02} \subset V_{0}^{-} \times V_{2}$ a Lagrangian subspace, and denote by

$$
\mathcal{T}_{\Lambda_{02}} \subset \operatorname{Lag}\left(V_{0}^{-} \times V_{1} \times V_{1}^{-} \times V_{2}\right)
$$

the subset of Lagrangian subspaces $\Lambda \subset V_{0}^{-} \times V_{1} \times V_{1}^{-} \times V_{2}$ with $\Lambda \pitchfork\left(V_{0} \times \Delta_{V_{1}} \times V_{2}\right)=$ : $\widehat{\Lambda}_{02}$ and $\pi_{02}\left(\widehat{\Lambda}_{02}\right)=\Lambda_{02}$. Then $\mathcal{T}_{\Lambda_{02}}$ is contractible.

Proof We fix metrics on $V_{0}, V_{1}$, and $V_{2}$. Then we will construct a contraction $\left(\rho_{t}\right)_{t \in[0,1]}, \rho_{t}: \mathcal{T}_{\Lambda_{02}} \rightarrow \mathcal{T}_{\Lambda_{02}}$ with $\rho_{0}=$ Id and $\rho_{1} \equiv \Psi\left(\Lambda_{02} \times\left(\Delta_{1}\right)^{\perp}\right)$, where $\Psi: V_{0}^{-} \times V_{2} \times V_{1} \times V_{1}^{-} \rightarrow V_{0}^{-} \times V_{1} \times V_{1}^{-} \times V_{2}$ exchanges the factors. To define $\rho_{t}(\Lambda)$ we write $\Lambda=\widehat{\Lambda}_{02} \oplus \widehat{\Lambda}_{11}$, where $\widehat{\Lambda}_{11}$ is the orthogonal complement of $\widehat{\Lambda}_{02}$ in $\Lambda$. Now $\widehat{\Lambda}_{02}$ is the image of $\left(\operatorname{Id}_{V_{0}}, i_{1}, i_{1}, \operatorname{Id}_{V_{2}}\right): \Lambda_{02} \rightarrow V_{0}^{-} \times V_{1} \times V_{1}^{-} \times V_{2}$ for a linear map $i_{1}: \Lambda_{02} \rightarrow V_{1}$ and $\widehat{\Lambda}_{11}$ is the image of $\left(j_{0}, \operatorname{Id}_{V_{1}}+j_{1},-\operatorname{Id}_{V_{1}}+j_{1}, j_{2}\right): V_{1} \rightarrow$ $V_{0}^{-} \times V_{1} \times V_{1}^{-} \times V_{2}$ for linear maps $j_{i}: V_{1} \rightarrow V_{i}$. One can check that

$$
\rho_{t}(\Lambda):=\operatorname{im}\left(\operatorname{Id}_{V_{0}}, t \cdot i_{1}, t \cdot i_{1}, \operatorname{Id}_{V_{2}}\right) \oplus \operatorname{im}\left(t \cdot j_{0}, \operatorname{Id}_{V_{1}}+t^{2} \cdot j_{1},-\operatorname{Id}_{V_{1}}+t^{2} \cdot j_{1}, t \cdot j_{2}\right)
$$

is an element of $\mathcal{T}_{\Lambda_{02}}$ for all $t \in[0,1]$ and defines a smooth contraction. 


\section{Floer cohomology}

The main content of this section is a review of the construction of graded Floer cohomology for pairs of Lagrangian submanifolds in monotone and exact cases by Floer, Oh, and Seidel. In Section 4.3 we then extend Floer cohomology to generalized Lagrangian correspondences, which in Section 5 will be reformulated in terms of pseudoholomorphic quilts.

\subsection{Monotonicity}

Let $(M, \omega)$ be a symplectic manifold. Let $\mathcal{J}(M, \omega)$ denote the space of compatible almost complex structures on $(M, \omega)$. Any $J \in \mathcal{J}(M, \omega)$ gives rise to a complex structure on the tangent bundle $T M$; the first Chern class $c_{1}(T M) \in H^{2}(M, \mathbb{Z})$ is independent of the choice of $J$. Throughout, we will use the following standing assumptions on all symplectic manifolds:

(M1) $(M, \omega)$ is monotone, that is for some $\tau \geq 0$

$$
[\omega]=\tau c_{1}(T M) .
$$

(M2) If $\tau>0$ then $M$ is compact. If $\tau=0$ then $M$ is (necessarily) noncompact but satisfies "bounded geometry" assumptions as in [33].

Note here that we treat the exact case $[\omega]=0$ as special case of monotonicity (with $\tau=0)$. Next, we denote the index map by

$$
c_{1}: \pi_{2}(M) \rightarrow \mathbb{Z}, \quad u \mapsto\left(c_{1}, u_{*}\left[S^{2}\right]\right) .
$$

The minimal Chern number $N_{M} \in \mathbb{N}$ is the nonnegative generator of its image.

Associated to a Lagrangian submanifold $L \subset M$ are the Maslov index and action (ie symplectic area) maps

$$
I: \pi_{2}(M, L) \rightarrow \mathbb{Z}, \quad A: \pi_{2}(M, L) \rightarrow \mathbb{R} .
$$

Our standing assumptions on all Lagrangian submanifolds are the following:

(L1) $L$ is monotone, that is

$$
2 A(u)=\tau I(u) \quad \forall u \in \pi_{2}(M, L)
$$

where the $\tau \geq 0$ is (necessarily) that from (M1).

(L2) $L$ is compact and oriented. 
Any homotopy class $[u] \in \pi_{2}(M, L)$ that is represented by a nontrivial $J$-holomorphic disk $u:(D, \partial D) \rightarrow(M, L)$ has positive action $A([u])=\int u^{*} \omega>0$. Monotonicity with $\tau>0$ then implies that the index is also positive. So, for practical purposes, we define the (effective) minimal Maslov number $N_{L} \in \mathbb{N}$ as the generator of $I(\{[u] \in$ $\left.\left.\pi_{2}(M, L) \mid A([u])>0\right\}\right) \subset \mathbb{N}$. If $M$ and $L$ are exact $(\tau=0)$, then $A \equiv 0$, so we have $N_{L}=\infty$.

If the Lagrangian submanifold $L$ is oriented then $I(u)$ is always even since it is the Maslov index of a loop of oriented Lagrangian subspaces. So the orientation and monotonicity assumption on $L$ imply $N_{L} \geq 2$, ie any nontrivial holomorphic disk must have $I(u) \geq 2$, which excludes disk bubbling in transverse moduli spaces of index 0 and 1.

In order for the Floer cohomology groups to be well defined we will also have to make the following additional assumption.

(L3) $L$ has minimal Maslov number $N_{L} \geq 3$.

Alternatively, following [22; 10], we may replace (L3) by an assumption on the disk counts, which we briefly recall here. Given any $p \in L$ and $\omega$-compatible almost complex structure $J \in \mathcal{J}(M, \omega)$ let $\mathcal{M}_{1}^{2}(L, J, p)$ be the moduli space of $J-$ holomorphic disks $u:(D, \partial D) \rightarrow(M, L)$ with Maslov number 2 and one marked point satisfying $u(1)=p$, modulo automorphisms of the disk fixing $1 \in \partial D$. Oh proves that for any $p \in L$ there exists a dense subset $\mathcal{J}^{\text {reg }}(p) \subset \mathcal{J}(M, \omega)$ such that $\mathcal{M}_{1}^{2}(L, J, p)$ is cut out transversely, and consequentially a finite set. Moreover, any relative spin structure on $L$ induces an orientation on $\mathcal{M}_{1}^{2}(L, J, p)$. Letting $\epsilon: \mathcal{M}_{1}^{2}(L, J, p) \rightarrow$ $\{ \pm 1\}$ denote the map comparing the given orientation to the canonical orientation of a point, the disk number of $L$,

$$
w(L):=\sum_{u \in \mathcal{M}_{1}^{2}(L, J, p)} \epsilon(u)
$$

is independent of $J \in J^{\text {reg }}(p)$ and $p \in L$. If we work without orientations then $w(L) \in \mathbb{Z}_{2}$ is still well defined.

As a third class of assumptions, we will restrict our considerations to Maslov coverings and gradings that are compatible with orientations, that is we make the following additional assumptions on the grading of the symplectic manifolds $M$ and Lagrangian submanifolds $L \subset M$. (In the case $N=2$ these assumptions reduce to (L2).)

(G1) $M$ is equipped with a Maslov covering $\operatorname{Lag}^{N}(M)$ for $N$ even, and the induced 2-fold Maslov covering $\operatorname{Lag}^{2}(M)$ is the one described in Example 3.0.8 (i).

(G2) $L$ is equipped with a grading $\sigma_{L}^{N}: L \rightarrow \operatorname{Lag}^{N}(M)$, and the induced 2-grading $L \rightarrow \operatorname{Lag}^{2}(M)$ is the one given by the orientation of $L$. 
In the following we discuss topological situations which ensure monotonicity.

Lemma 4.1.1 Suppose that $M$ is monotone and $L \subset M$ is a compact Lagrangian such that $\pi_{1}(L)$ is torsion; that is every element has finite order, and there is a finite maximal order $k$. Then $L$ is monotone and the minimal Maslov number is at least $2 N_{M} / k$.

Proof Let $u:(D, \partial D) \rightarrow(M, L)$ and let $k(u)$ be the order of the restriction of $u$ to the boundary in $\pi_{1}(L)$. After passing to a $k(u)$-fold branched cover $\tilde{u}$, we may assume that the restriction of $\tilde{u}$ to $\partial D$ is homotopically trivial in $L$. By adding the homotopy we obtain a sphere $v: S^{2} \rightarrow M$ with $k(u) I(u)=I(\tilde{u})=2 c_{1}(v)$ divisible by $2 N_{M}$. For the relation between the first Chern class and the Maslov index, see eg McDuff and Salamon [18, Appendix C]. The similar identity for the actions (due to $\left.\omega\right|_{L}=0$ ) completes the proof.

In practice, we will need the action-index relation not only for disks as in (L1) but also for other surfaces with several boundary components mapping to several Lagrangians. (This really only becomes relevant in [41] for the definition of relative invariants from surfaces with strip-like ends.) In particular, to define Floer cohomology for a pair of Lagrangians (and especially later to prove the isomorphism (3)) we need the actionindex for annuli between the two Lagrangians. This provides the energy-index relation in Remark 4.2.2. In fact, it also implies monotonicity (L1) for both Lagrangians as long as $M$ is connected.

Definition 4.1.2 (a) We say that a tuple $\left(L_{e}\right)_{e \in \mathcal{E}}$ is monotone with monotonicity constant $\tau \geq 0$ if the following holds: Let $\Sigma$ be any connected compact surface with nonempty boundary $\partial \Sigma=\bigsqcup_{e \in \mathcal{E}} C_{e}$ (with $C_{e}$ possibly empty or disconnected). Then for every map $u: \Sigma \rightarrow M$ satisfying $u\left(C_{e}\right) \subset L_{e}$ we have the action-index relation

$$
2 \int u^{*} \omega=\tau \cdot I\left(u^{*} T M,\left(u^{*} T L_{e}\right)_{e \in \mathcal{E}}\right),
$$

where $I$ is the sum of the Maslov indices of the totally real subbundles $\left(\left.u\right|_{C_{e}}\right)^{*} T L_{e}$ in some fixed trivialization of $u^{*} T M$.

(b) We say that a pair $\left(L_{0}, L_{1}\right)$ is monotone for Floer theory if (a) holds for the annulus $\Sigma=[0,1] \times S^{1}$ and every map $u$ with boundary values $u\left(\{j\} \times S^{1}\right) \subset L_{j}$ for $j=0,1$.

The following is a minor generalization of [22, Proposition 2.7]. 


\section{Lemma 4.1.3 Suppose that $M$ is monotone.}

(a) If each $L_{e} \subset M$ is monotone, and the image of each $\pi_{1}\left(L_{e}\right)$ in $\pi_{1}(M)$ is torsion, then the tuple $\left(L_{e}\right)_{e \in \mathcal{E}}$ is monotone.

(b) If both $L_{0}, L_{1} \subset M$ are monotone, and the image of $\pi_{1}\left(L_{0}\right)$ or $\pi_{1}\left(L_{1}\right)$ in $\pi_{1}(M)$ is torsion, then the pair $\left(L_{0}, L_{1}\right)$ is monotone for Floer theory.

Proof To check (a) consider $u$ : $\Sigma \rightarrow M$ satisfying $u\left(C_{e}\right) \subset L_{e}$. By assumption we have integers $N_{e} \in \mathbb{N}$ such that $\left.N_{e} u\right|_{C_{e}}$ is contractible in $M$. Let $N=\prod_{e \in \mathcal{E}} N_{e}$, so that $\left.N u\right|_{C_{e}}$ is contractible for all boundary components $C_{e}$ of $\Sigma$. Let $\widetilde{\Sigma} \rightarrow \Sigma$ be a ramified $N$-cover defined as follows: Pick one ramification point $z_{1}, \ldots, z_{k} \in \Sigma$ in each connected component of $\Sigma$ with nonempty boundary. Then there exists a representation $\rho: \pi_{1}\left(\Sigma \backslash\left\{z_{1}, \ldots, z_{k}\right\}\right) \rightarrow \mathbb{Z}_{N}$ with $\rho\left(\left[C_{e}\right]\right)=\left[N / N_{e}\right]$. The induced ramified cover $\rho: \widetilde{\Sigma} \rightarrow \Sigma$ satisfies the following: The inverse image $\widetilde{C}_{e}$ of $C_{e}$ consists of $N / N_{e}$ connected components, each of which is an $N_{e}$-fold cover of $C_{e}$. Now the pullback $\tilde{u}: \tilde{\Sigma} \rightarrow M$ of $u: \Sigma \rightarrow M$ has restrictions to the boundary $\left.\tilde{u}\right|_{\tilde{C}_{e}}$ that are homotopically trivial in $M$. Thus $\tilde{u}$ is homotopic to the connected sum of some maps $v_{e, j}:(D, \partial D) \rightarrow\left(M, L_{e}\right)$ for $j=1, \ldots, N / N_{e}$ and a map $v: S \rightarrow M$ on a closed surface $S$. We can now use the closedness of $\omega$ and the monotonicity of $M$ and each $L_{e}$ to deduce

$$
\begin{aligned}
2 N \int_{\Sigma} u^{*} \omega=2 \int_{\tilde{\Sigma}} \tilde{u}^{*} \omega & =2 \int_{S} v^{*} \omega+\sum_{\substack{e \in \mathcal{E}, j=1, \ldots, N / N_{e}}} 2 \int_{D} v_{e, j}^{*} \omega \\
& =2 \tau c_{1}\left(v^{*} T M\right)+\sum_{\substack{e \in \mathcal{E} \\
j=1, \ldots, N / N_{e}}} \tau I\left(v_{e, j}\right)=\tau I(\widetilde{u})=\tau N I(u),
\end{aligned}
$$

using properties of the Maslov index explained in [18, Appendix C]. The first equality $N \int u^{*} \omega=\int \tilde{u}^{*} \omega$ can be confirmed by integrating over the complement of the branch points. The last equality $I(\widetilde{u})=N I(u)$ holds since any trivialization $u^{*} T M \cong \Sigma \times \mathbb{C}^{n}$ induces a trivialization $\widetilde{u}^{*} T M \cong \widetilde{\Sigma} \times \mathbb{C}^{n}$, which restricts to the $N$-fold covering $\rho \times \mathrm{Id}: \widetilde{C}_{e} \times \mathbb{C}^{n} \rightarrow C_{e} \times \mathbb{C}^{n}$ on each boundary component.

In the case of (b) we can take a multiple cover of the annulus such that one boundary loop is contractible in $M$, and hence the multiply covered annulus is homotopic to two disks to which we can apply monotonicity of the single Lagrangians.

In the exact case, with $\omega=d \lambda$, any tuple of exact Lagrangians $\left(L_{e}\right)_{e \in \mathcal{E}}$, that is with $\left[\left.\lambda\right|_{L_{e}}\right]=0 \in H^{1}\left(L_{e}\right)$, is automatically monotone. Moreover, note that monotonicity is invariant under Hamiltonian isotopies of one or several Lagrangians. 
Remark 4.1.4 Another situation in which one naturally has monotonicity is the BohrSommerfeld setting, as pointed out to us by P Seidel. Suppose that the cohomology class $[\omega]$ is integral. Let $(\mathcal{L}, \nabla) \rightarrow(M, \omega)$ be a unitary line-bundle-with-connection having curvature $(2 \pi / i) \omega$. The restriction of $(\mathcal{L}, \nabla)$ to any Lagrangian $L \subset M$ is flat. $L$ is Bohr-Sommerfeld if the restriction of $(\mathcal{L}, \nabla)$ to $L$ is trivial, that is, there exists a nonzero horizontal section. In that case, we choose a horizontal section $\phi_{L}^{\mathcal{L}}$ of unit length, which is unique up to a collection of phases $U(1)^{\pi_{0}(L)}$. Suppose that $M$ is monotone, $[\omega]=\lambda c_{1}(M)$ for some $\lambda>0$. Since $c_{1}(M)$ and $[\omega]$ are integral, we must have $\lambda=k / l$ for some integers $k, l>0$. Let $\mathcal{K}^{-1} \rightarrow M$ denote the anticanonical bundle, $\mathcal{K}_{m}^{-1}=\Lambda_{\mathbb{C}}^{\text {top }}\left(T_{m}^{0,1} M\right)$, which satisfies $k c_{1}\left(\mathcal{K}^{-1}\right)=l \frac{i}{2 \pi}[\operatorname{curv}(\nabla)]=l c_{1}(\mathcal{L})$. Hence there exists an isomorphism

$$
\Phi:\left(\mathcal{K}^{-1}\right)^{\otimes k} \rightarrow \mathcal{L}^{\otimes l}
$$

Let $L \subset M$ be an oriented Lagrangian submanifold. The restriction of $\mathcal{K}^{-1}$ to $L$ has a natural nonvanishing section $\phi_{L}^{\mathcal{K}}$ given by the orientation and the isomorphisms

$$
\left.\Lambda_{\mathbb{R}}^{\text {top }} T L \rightarrow \Lambda_{\mathbb{C}}^{\text {top }} T^{0,1} M\right|_{L}, \quad v_{1} \wedge \cdots \wedge v_{n} \mapsto\left(v_{1}+i J v_{1}\right) \wedge \cdots \wedge\left(v_{n}+i J v_{n}\right) .
$$

We say that $L$ is Bohr-Sommerfeld monotone with respect to $(\mathcal{L}, \nabla, \Phi)$ if it is BohrSommerfeld and the section $\left(\phi_{L}^{\mathcal{L}}\right)^{\otimes l}$ is homotopic to $\Phi \circ\left(\phi_{L}^{\mathcal{K}}\right)^{\otimes k}$, that is, there exists a function $\psi: L \rightarrow \mathbb{R}$ such that

$$
\left(\exp (2 \pi i \psi) \phi_{L}^{\mathcal{L}}\right)^{\otimes l}=\Phi \circ\left(\phi_{L}^{\mathcal{K}}\right)^{\otimes k} .
$$

Lemma 4.1.5 Let $\left(L_{e}\right)_{e \in \mathcal{E}}$ be a collection of Lagrangians such that each is BohrSommerfeld monotone with respect to $(\mathcal{L}, \nabla, \Phi)$. Then $\left(L_{e}\right)_{e \in \mathcal{E}}$ is monotone.

Proof Let $\Sigma$ be a compact Riemann surface with boundary components $\left(C_{e}\right)_{e \in \mathcal{E}}$. Let $u: \Sigma \rightarrow M$ be a map with boundary $u\left(C_{e}\right) \subset L_{e}$. The index $I(u)$ is the sum of Maslov indices of the bundles $\left(\left.u\right|_{C_{e}}\right)^{*} T L_{e}$, with respect to some fixed trivialization of $u^{*} T M$. Equivalently, $I(u)$ is the sum of winding numbers of the sections $\phi_{L_{e}}^{\mathcal{K}}$ with respect to the induced trivialization of $u^{*} \mathcal{K}^{-1}$. Since each $L_{e}$ is Bohr-Sommerfeld, $k I(u)$ is the sum of the winding numbers of the sections $\left(\phi_{L_{e}}^{\mathcal{L}}\right)^{\otimes l}$, with respect to the induced trivialization of $u^{*} \mathcal{L}^{\otimes l}$. Write $u^{*} \nabla^{\otimes l}=\mathrm{d}+\alpha$ for some $\alpha \in \Omega^{1}(\Sigma)$ in this trivialization, so that $u^{*} \operatorname{curv}\left(\nabla^{\otimes l}\right)=\mathrm{d} \alpha$. Since the sections are horizontal, we have

$$
k I(u)=(i / 2 \pi) \int_{\partial \Sigma} \alpha=(i / 2 \pi) \int_{\Sigma} u^{*} \operatorname{curv}\left(\nabla^{\otimes l}\right)=l A(u) .
$$




\subsection{Graded Floer cohomology for pairs of Lagrangians}

Let $L_{0}, L_{1} \subset M$ be compact Lagrangian submanifolds. For a time-dependent Hamiltonian $H \in C^{\infty}([0,1] \times M)$ let $\left(X_{t}\right)_{t \in[0,1]}$ denote the family of Hamiltonian vector fields for $\left(H_{t}\right)_{t \in[0,1]}$, and let $\phi_{t_{0}, t_{1}}: M \rightarrow M$ denote its flow. (That is, $\phi_{t_{0}, t_{1}}(y)=$ $x\left(t_{1}\right)$, where $x:[0,1] \rightarrow M$ satisfies $\dot{x}=X_{t}(x), x\left(t_{0}\right)=y$.) We will abbreviate $\phi_{1}:=\phi_{0,1}$ for the time 1 flow from $t_{0}=0$ to $t_{1}=1$. Let $\operatorname{Ham}\left(L_{0}, L_{1}\right)$ be the set of $H \in C^{\infty}([0,1] \times M)$ such that $\phi_{1}\left(L_{0}\right)$ intersects $L_{1}$ transversally. Then we have a finite set of perturbed intersection points

$$
\mathcal{I}\left(L_{0}, L_{1}\right) ;=\left\{\gamma:[0,1] \rightarrow M \mid \gamma(t)=\phi_{0, t}(\gamma(0)), \gamma(0) \in L_{0}, \gamma(1) \in L_{1}\right\} .
$$

It is isomorphic to the intersection $\phi_{1}\left(L_{0}\right) \pitchfork L_{1}$. If we assume that $M$ and $L_{0}, L_{1}$ are graded as in $(\mathrm{G} 1)-(\mathrm{G} 2)$, then we obtain a degree map from Section 3,

$$
\mathcal{I}\left(L_{0}, L_{1}\right) \rightarrow \mathbb{Z}_{N}, \quad x \mapsto|x|=d\left(\sigma_{L_{0}}^{N}(x), \sigma_{L_{1}}^{N}(x)\right) .
$$

Since $N$ is even the sign $(-1)^{|x|}$ is well-defined. It agrees with the usual sign in the intersection number, given by the orientations of $\phi_{1}\left(L_{0}\right)$ and $L_{1}$, which also determine the mod 2 grading by assumption.

We denote the space of time-dependent $\omega$-compatible almost complex structures by

$$
\mathcal{J}_{t}(M, \omega):=C^{\infty}([0,1], \mathcal{J}(M, \omega)) .
$$

For any $J \in \mathcal{J}_{t}(M, \omega)$ and $H \in \operatorname{Ham}\left(L_{0}, L_{1}\right)$ we say that a map $u: \mathbb{R} \times[0,1] \rightarrow M$ is $(J, H)$-holomorphic with Lagrangian boundary conditions if

$$
\begin{gathered}
\bar{\partial}_{J, H} u:=\partial_{s} u(s, t)+J_{t, u(s, t)}\left(\partial_{t} u(s, t)-X_{t}(u(s, t))\right)=0, \\
u(\mathbb{R}, 0) \subset L_{0}, \quad u(\mathbb{R}, 1) \subset L_{1} .
\end{gathered}
$$

The (perturbed) energy of a solution is

$$
E_{H}(u):=\int_{\mathbb{R} \times[0,1]}\left|\partial_{s} u\right|^{2}=\int_{\mathbb{R} \times[0,1]} u^{*} \omega+\mathrm{d}(H(u) \mathrm{d} t) .
$$

The following exponential decay lemma of Floer [8] will be needed later and is part of the proof of Theorem 4.2.3 below.

Lemma 4.2.1 Let $H \in \operatorname{Ham}\left(L_{0}, L_{1}\right)$ and $J \in \mathcal{J}_{t}(M, \omega)$. Then for any $(J, H)-$ holomorphic strip $u: \mathbb{R} \times[0,1] \rightarrow M$ with Lagrangian boundary conditions in $L_{0}, L_{1}$ the following are equivalent:

(a) $u$ has finite energy $E_{H}(u)=\int_{\mathbb{R} \times[0,1]}\left|\partial_{s} u\right|^{2}<\infty$.

(b) There exist $x_{ \pm} \in \mathcal{I}\left(L_{0}, L_{1}\right)$ such that $u(s, \cdot)$ converges to $x_{ \pm}$exponentially in all derivatives as $s \rightarrow \pm \infty$. 
For any $x_{ \pm} \in \mathcal{I}\left(L_{0}, L_{1}\right)$ we denote by

$\mathcal{M}\left(x_{-}, x_{+}\right):=\left\{u: \mathbb{R} \times[0,1] \rightarrow M \mid(20),(21), E_{H}(u)<\infty, \lim _{s \rightarrow \pm \infty} u(s, \cdot)=x_{ \pm}\right\} / \mathbb{R}$

the space of finite energy $(J, H)$-holomorphic maps modulo translation in $s \in \mathbb{R}$. It is isomorphic to the moduli space of finite energy $J^{\prime}$-holomorphic maps with boundary conditions in $\phi_{1}\left(L_{0}\right)$ and $L_{1}$, and without Hamiltonian perturbation. Here $J^{\prime} \in \mathcal{J}_{t}(M, \omega)$ arises from $J$ by pullback with $\phi_{t, 1}$.

Remark 4.2.2 Suppose that the pair $\left(L_{0}, L_{1}\right)$ is monotone, then for any $x_{ \pm} \in$ $\mathcal{I}\left(L_{0}, L_{1}\right)$ there exists a constant $c\left(x_{-}, x_{+}\right)$such that for all $u \in \mathcal{M}\left(x_{-}, x_{+}\right)$the energy-index relation holds:

$$
2 E_{H}(u)=\tau \cdot \operatorname{Ind}\left(D_{u}\right)+c\left(x_{-}, x_{+}\right),
$$

where $D_{u}$ denotes the linearized operator at $u$ of the Cauchy-Riemann Equation (20) on the space of sections of $u^{*} T M$ satisfying the linearized Lagrangian boundary conditions from (21). Its Fredholm index is given by the Maslov-Viterbo index of $u$. This monotonicity ensures energy bounds for the moduli spaces of fixed index and thus compactness up to bubbling.

Theorem 4.2.3 (Floer, Oh) Let $L_{0}, L_{1} \subset M$ be a monotone pair ${ }^{4}$ of Lagrangian submanifolds satisfying (L1)-(L2) and (M1)-(M2). For any $H \subset \operatorname{Ham}\left(L_{0}, L_{1}\right)$ there exists a dense subset $\mathcal{J}_{t}^{\text {reg }}\left(L_{0}, L_{1} ; H\right) \subset \mathcal{J}_{t}(M, \omega)$ such that the following holds for all $x_{ \pm} \in \mathcal{I}\left(L_{0}, L_{1}\right)$.

(a) $\mathcal{M}\left(x_{-}, x_{+}\right)$is a smooth manifold whose dimension near a nonconstant solution $u$ is given by the formal dimension $\operatorname{Ind}\left(D_{u}\right)-1$. We denote $\mathcal{M}\left(x_{-}, x_{+}\right)_{j}:=$ $\left\{\operatorname{Ind}\left(D_{u}\right)=j+1\right\}$; thus excluding the constant solution from $\mathcal{M}(x, x)_{0}$.

(b) The component $\mathcal{M}\left(x_{-}, x_{+}\right)_{0} \subset \mathcal{M}\left(x_{-}, x_{+}\right)$of formal dimension zero is finite.

(c) Suppose that $L_{0}$ and $L_{1}$ have minimal Maslov numbers $N_{L_{k}} \geq 3$. Then the one-dimensional component $\mathcal{M}\left(x_{-}, x_{+}\right){ }_{1} \subset \mathcal{M}\left(x_{-}, x_{+}\right)$has a compactification as one-dimensional manifold with boundary

$$
\partial \overline{\mathcal{M}\left(x_{-}, x_{+}\right)_{1}} \cong \bigcup_{x \in \mathcal{I}\left(L_{0}, L_{1}\right)} \mathcal{M}\left(x_{-}, x\right)_{0} \times \mathcal{M}\left(x, x_{+}\right)_{0} .
$$

(d) If $\left(L_{0}, L_{1}\right)$ is relatively spin (as defined in eg [40]), then there exists a coherent set of orientations on $\mathcal{M}\left(x_{-}, x_{+}\right)_{0}, \mathcal{M}\left(x_{-}, x_{+}\right)_{1}$ for all $x_{ \pm} \in \mathcal{I}\left(L_{0}, L_{1}\right)$, that is, orientations compatible with (23).

\footnotetext{
${ }^{4}$ Throughout, we are working with monotone pairs of Lagrangians in the sense of Definition 4.1.2.
} 
For the proofs of (a)-(c) we refer to Oh's paper [22] and the clarifications [23; 15]. For the exact case see Seidel [33]. The proof of (d) is contained in our paper [40] loosely following Fukaya et al [10]. From (d) we obtain a map

$$
\epsilon: \mathcal{M}\left(x_{-}, x_{+}\right)_{0} \rightarrow\{ \pm 1\}
$$

defined by comparing the given orientation to the canonical orientation of a point.

Now let $M$ be a monotone symplectic manifold satisfying (M1)-(M2) and equipped with an $N$-fold Maslov covering. Let $L_{0}, L_{1} \subset M$ be a monotone, relative spin pair of graded Lagrangian submanifolds satisfying (L1)-(L3), and let $H \in \operatorname{Ham}\left(L_{0}, L_{1}\right)$. The Floer cochain group is the $\mathbb{Z}_{N}$-graded group

$$
C F\left(L_{0}, L_{1}\right)=\bigoplus_{d \in \mathbb{Z}_{N}} C F^{d}\left(L_{0}, L_{1}\right), \quad C F^{d}\left(L_{0}, L_{1}\right)=\bigoplus_{\substack{x \in \mathcal{I}\left(L_{0}, L_{1}\right) \\|x|=d}} \mathbb{Z}\langle x\rangle,
$$

and the Floer coboundary operator is the map of degree 1,

$$
\begin{aligned}
\partial^{d} & : C F^{d}\left(L_{0}, L_{1}\right) \rightarrow C F^{d+1}\left(L_{0}, L_{1}\right), \\
\partial^{d}\left\langle x_{-}\right\rangle & :=\sum_{x_{+} \in \mathcal{I}\left(L_{0}, L_{1}\right)}\left(\sum_{u \in \mathcal{M}\left(x_{-}, x_{+}\right)_{0}} \epsilon(u)\right)\left\langle x_{+}\right\rangle .
\end{aligned}
$$

defined by

Here we choose some $J \in \mathcal{J}_{t}^{\text {reg }}\left(L_{0}, L_{1} ; H\right)$. If an isolated trajectory $u \in \mathcal{M}\left(x_{-}, x_{+}\right)_{0}$ exists, then the degree identity $\left|x_{+}\right|=\left|x_{-}\right|+1$ can be seen by concatenating the paths $\tilde{\gamma_{0}}, \tilde{\gamma_{1}}$ of graded Lagrangians in the definition of $\left|x_{-}\right|$with the unique graded lifts of $u^{*} T L_{0}, u^{*} T L_{1}$ to obtain paths of graded Lagrangians defining $\left|x_{+}\right|$(using a trivialization of $u^{*} T M$ over the strip, compactified to a disk). By additivity of the Maslov index this shows $\left|x_{+}\right|=\left|x_{-}\right|+I\left(u^{*} T L_{0}, u^{*} T L_{1}\right)=\left|x_{-}\right|+1$. It follows from Theorem 4.2.3 that $\partial^{2}=0$. Alternatively, following [22], we could drop assumption (L3), then $\partial^{2}=\left(w\left(L_{0}\right)-w\left(L_{1}\right)\right)$ Id, and to obtain a well defined cohomology it suffices to assume that this disk count vanishes. In either case, the Floer cohomology ${ }^{5}$

$$
H F\left(L_{0}, L_{1}\right):=\bigoplus_{d \in \mathbb{Z}_{N}} H F^{d}\left(L_{0}, L_{1}\right), \quad H F^{d}\left(L_{0}, L_{1}\right):=\operatorname{ker}\left(\partial^{d}\right) / \operatorname{im}\left(\partial^{d-1}\right)
$$

is $\mathbb{Z}_{N}$-graded. It is independent of the choice of $H$ and $J$; a generalization of this fact is proved in Section 5.3 below. If the gradings moreover satisfy (G1)-(G2), then we have a well defined splitting

$$
H F\left(L_{0}, L_{1}\right)=H F^{\text {even }}\left(L_{0}, L_{1}\right) \oplus H F^{\text {odd }}\left(L_{0}, L_{1}\right),
$$

which coincides with the splitting induced by the orientations of $L_{0}, L_{1} \subset M$.

${ }^{5}$ Note that our conventions differ from Seidel's definition of graded Floer cohomology in [31] in two points which cancel each other: The roles of $x_{-}$and $x_{+}$are interchanged and we switched the sign of the Maslov index in the definition of the degree (11). 


\subsection{Floer cohomology for generalized Lagrangian correspondences}

The goal of this section is to define a first version of Floer cohomology for a cyclic generalized Lagrangian correspondence $\underline{L}$ as in Definition 2.1.3. So we consider $\underline{L}=$ $\left(L_{01}, \ldots, L_{r(r+1)}\right)$, a sequence of smooth Lagrangian correspondences $L_{(j-1) j} \subset$ $M_{j-1}^{-} \times M_{j}$ between a sequence $M_{0}, M_{1}, \ldots, M_{r+1}=M_{0}$ of symplectic manifolds. For example, we could consider a noncyclic sequence of Lagrangians $L_{01} \subset M_{1}$, $\left(L_{(i-1) i} \subset M_{i-1}^{-} \times M_{i}\right)_{i=2, \ldots, r}, L_{r 0} \subset M_{r}^{-}$, which is a special case of the cyclic setup with $M_{0}=\{\mathrm{pt}\}$. The usual Floer cohomology for pairs of Lagrangians fits into this case with $r=1$ and Lagrangian submanifolds $L_{01}, L_{10} \subset M_{1}$.

We assume that $\underline{L}$ satisfies (M1)-(M2) and (L1)-(L3), ie each $M_{j}$ satisfies (M1)-(M2) and each $L_{(j-1) j}$ satisfies (L1)-(L3) with a fixed monotonicity constant $\tau \geq 0$. We moreover assume that $\underline{L}$ is graded in the sense of Definition 3.1.2 and equipped with a relative spin structure in the following sense. Alternatively, we may replace the minimal Maslov assumption (L3) by the assumption that the sum of disk numbers from (18) vanishes:

$$
w\left(L_{01}\right)+\cdots+w\left(L_{r(r+1)}\right)=0 .
$$

Definition 4.3.1 Let $\underline{L}=\left(L_{01}, \ldots, L_{r(r+1)}\right)$ be a cyclic generalized Lagrangian correspondence (ie $L_{j(j+1)} \subset M_{j}^{-} \times M_{j+1}$ for a cyclic sequence $M_{0}, M_{1}, \ldots, M_{r+1}=$ $M_{0}$ of symplectic manifolds). A relative spin structure on $\underline{L}$ consists of a collection of background classes $b_{j} \in H^{2}\left(M_{j}, \mathbb{Z}_{2}\right)$ for $j=0, \ldots, r+1$ and relative spin structures on $L_{j(j+1)}$ with background classes $-\pi_{j}^{*} b_{j}+\pi_{j+1}^{*} b_{j+1}$. The cyclic requirement on the background classes $b_{0} \in H^{2}\left(M_{0}, \mathbb{Z}_{2}\right)$ and $b_{r+1} \in H^{2}\left(M_{r+1}, \mathbb{Z}_{2}\right)=H^{2}\left(M_{0}, \mathbb{Z}_{2}\right)$ is $b_{r+1}=b_{0}$ for $r$ odd and $b_{r+1}=b_{0}+w_{2}\left(M_{0}\right)$ for $r$ even. ${ }^{6}$

Eventually, in Section 5, we will define the Floer cohomology $H F(\underline{L})$ directly, using "quilts of pseudoholomorphic strips". In this section however we define $H F(\underline{L})$ as a special case of the Floer cohomology for pairs of Lagrangian submanifolds - which are constructed from the sequence $\underline{L}$ as follows. If $\underline{L}$ has even length $r+1 \in 2 \mathbb{N}$ we define a pair of graded Lagrangian submanifolds,

$$
\begin{aligned}
& L_{(0)}:=\left(L_{01} \times L_{23} \times \cdots \times L_{(r-1) r}\right) \quad \subset M_{0}^{-} \times M_{1} \times M_{2}^{-} \times \cdots \times M_{r}=: \widetilde{M} . \\
& L_{(1)}:=\left(L_{12} \times L_{34} \times \cdots \times L_{r(r+1)}\right)^{T} \quad
\end{aligned}
$$

Here we denote by $M_{1}^{-} \times \cdots \times M_{r}^{-} \times M_{0} \rightarrow M_{0}^{-} \times M_{1} \times \cdots \times M_{r}, Z \mapsto Z^{T}$ the transposition of the last to the first factor, combined with an overall sign change in

${ }^{6}$ This shift is necessary in order to fit in the canonical relative spin structure for the diagonal $\Delta_{0}$; see our paper [39] for details. 
the symplectic form. If $\underline{L}$ has odd length $r+1 \in 2 \mathbb{N}+1$ we insert the diagonal $\Delta_{0} \subset M_{0}^{-} \times M_{0}=M_{r+1}^{-} \times M_{0}$ (equipped with its canonical grading) into $\underline{L}$ before arranging it into a pair of Lagrangian submanifolds as above, yielding

$$
\begin{aligned}
& L_{(0)}=\left(L_{01} \times L_{23} \times \cdots \times L_{r(r+1)}\right) \\
& L_{(1)}=\left(L_{12} \times L_{34} \times \cdots \times L_{(r-1) r} \times \Delta_{0}\right)^{T} \quad \subset M_{0}^{-} \times M_{1} \times \cdots \times M_{r}^{-} \times M_{r+1}=\widetilde{M} .
\end{aligned}
$$

In the case of a noncyclic correspondence with $M_{0}=M_{r+1}=\{\mathrm{pt}\}$ the transposition as well as insertion of the diagonal are trivial operations. Note that, beyond the grading, also the monotonicity, compactness, and orientation assumptions (L1)-(L2) on $\underline{L}$ transfer directly to properties (L1)-(L2) for $L_{(0)}$ and $L_{(1)}$. Similarly, a relative spin structure on $\underline{L}$ induces compatible relative spin structures on $L_{(0)}$ and $L_{(1)}$ [40]. Moreover, we say that $\underline{L}$ is monotone if the pair of Lagrangians $\left(L_{(0)}, L_{(1)}\right)$ is monotone in the sense of Definition 4.1.2(b). If this is the case, then a graded Floer cohomology for $\underline{L}$ can be defined by

$$
H F(\underline{L}):=H F\left(L_{(0)}, L_{(1)}\right) .
$$

Remark 4.3.2 To see that $H F\left(L_{(0)}, L_{(1)}\right)$ is well defined we need to make sure that $\partial^{2}=0$. This holds immediately if $L_{(0)}$ and $L_{(1)}$ also satisfy (L3), if the bubbling of holomorphic discs is otherwise excluded, or if the effect of bubbling sums up to zero. This can be achieved if all Lagrangians satisfy (L3) or, weaker, if the total disk count vanishes (24).

(a) Note that the assumption (L3) on the factors of $\underline{L}$ does not directly transfer to the product Lagrangians $L_{(0)}$ and $L_{(1)}$ since a difference of Maslov numbers greater than 3 could give a total Maslov number less than 3 . However, if we use a split almost complex structure $\widetilde{J}=J_{0} \oplus \cdots \oplus J_{r}$ on $\widetilde{M}$, induced from compatible almost complex structures $J_{k}$ on each $M_{k}$, then any nonconstant holomorphic disc in $\widetilde{M}$ with boundary on $L_{(0)}$ or $L_{(1)}$ will simply be a product of $J_{k}$-holomorphic discs. Pairs of these discs take boundary values in the Lagrangian correspondences $L_{(k-1) k}$ which satisfy the monotonicity assumptions as well as (L3). Hence each of these double discs must have nonnegative area and hence index, and at least one of them has positive area and hence Maslov index at least 3. This excludes bubbling in moduli spaces of index 1 or 2 , hence proves $\partial^{2}=0$.

The proof that transversality can be achieved with an almost complex structure (and also Hamiltonian perturbation) of split type can be found in Theorem 5.2.4 and Proposition 5.2.1 below. This excludes bubbling such that $\partial^{2}=0$ for this specific choice of perturbation data (and hence for any other choice of regular perturbation data). So the Floer cohomology $H F\left(L_{(0)}, L_{(1)}\right)$ is indeed well defined. 
(b) In the absence of (L3) we have $\partial^{2}=\left(w\left(L_{(0)}\right)-w\left(L_{(1)}\right)\right)$ Id by [22]. Using a split almost complex structure we show in [43] (here stated in the case of even length $r+1)$ that $w\left(L_{(0)}\right)=w\left(L_{01}\right)+w\left(L_{23}\right)+\cdots+w\left(L_{(r-1) r}\right)$ and $w\left(L_{(1)}\right)=$ $-w\left(L_{12}\right)-w\left(L_{34}\right)-\cdots-w\left(L_{r(r+1)}\right)$, hence $\partial^{2}=\left(w\left(L_{01}\right)+\cdots+w\left(L_{r(r+1)}\right)\right) \operatorname{Id}$, which vanishes if we assume (24). The relative minus sign in $w\left(L_{(1)}\right)$ arises from the fact that eg $w\left(L_{12}\right)$ is the disk count for the Lagrangian $L_{12} \subset M_{1}^{-} \times M_{2}$, whereas in the construction of $L_{(1)}$ we use $L_{12}^{-} \subset M_{1} \times M_{2}^{-}$, the same submanifold but viewed as Lagrangian with respect to the reversed symplectic structure. The disk counts are related by $w\left(L_{12}^{-}\right)=-w\left(L_{12}\right)$, since the $\left(J_{1},-J_{2}\right)$-holomorphic discs with boundary on $L_{12}^{-}$are identified with $\left(-J_{1}, J_{2}\right)$-holomorphic discs with boundary on $L_{12}$ via a reflection of the domain, which is orientation reversing for the moduli spaces.

In the case of a noncyclic sequence the Floer cohomology $H F(\underline{L})$ specializes to

$$
H F\left(L_{1}, L_{12}, \ldots, L_{(r-1) r}, L_{r}\right)=H F\left(L_{1} \times L_{23} \times \cdots, L_{12} \times L_{34} \times \cdots\right) .
$$

In particular we reproduce the definition of Floer cohomology for a pair of Lagrangians $L_{0}, L_{1} \subset M$, viewed as cyclic correspondence

$$
\{\mathrm{pt}\} \stackrel{L_{0}}{\longrightarrow} M \stackrel{L_{1}}{\longrightarrow}\{\mathrm{pt}\} .
$$

We moreover define a Floer cohomology for any Lagrangian $L \subset M^{-} \times M$, viewed as cyclic correspondence $M \stackrel{L}{\rightarrow} M$, in particular for graphs $L=\operatorname{graph}(\phi)$ of symplectomorphisms $\phi: M \rightarrow M$. By definition, this invariant is $H F(L):=H F\left(L, \Delta_{M}\right)$, which reproduces the Floer cohomology $H F(\operatorname{graph}(\phi))=H F\left(\operatorname{graph}(\phi), \Delta_{M}\right)=H F(\phi)$ of a symplectomorphism.

\section{Quilted Floer cohomology}

The purpose of this section is to reformulate the definition of Floer cohomology for generalized Lagrangian correspondences in terms of quilted surfaces (consisting of strips). As in Section 4.3 consider a cyclic generalized Lagrangian correspondence $\underline{L}$, that is, a sequence of symplectic manifolds $M_{0}, M_{1}, \ldots, M_{r}, M_{r+1}$ with $M_{0}=M_{r+1}$ for $r \geq 0$, and a sequence of Lagrangian correspondences

$$
L_{01} \subset M_{0}^{-} \times M_{1}, \quad L_{12} \subset M_{1}^{-} \times M_{2}, \ldots, \quad L_{r(r+1)} \subset M_{r}^{-} \times M_{r+1} .
$$

\subsection{Unfolding of Floer cohomology in products}

We defined the Floer cohomology $H F(\underline{L})$ as the standard Floer cohomology in the product manifold $\widetilde{M}=M_{0}^{-} \times M_{1} \times M_{2}^{-} \times \cdots$ of a pair of Lagrangians $L_{(0)}, L_{(1)}$ 
that is built from the cyclic sequence $\underline{L}$. We will show how quilts arise naturally from "unfolding" this construction and phrasing it in terms of tuples of holomorphic curves in the $M_{j}$.

Informally, $H F(\underline{L})$ can be viewed as the Morse homology on the path space

$$
\mathcal{P}\left(L_{(0)}, L_{(1)}\right)=\left\{y:[0,1] \rightarrow \widetilde{M} \mid y(0) \in L_{(0)}, y(1) \in L_{(1)}\right\}
$$

of the (potentially multivalued) symplectic action functional

$$
\mathcal{A}_{H}(y)=\int_{[0,1] \times[0,1]} v^{*} \omega_{\widetilde{M}}+\int_{0}^{1} H(t, y(t)) \mathrm{d} t .
$$

Here $v:[0,1] \rightarrow \mathcal{P}\left(L_{(0)}, L_{(1)}\right)$ is a smooth homotopy from a fixed $v(0)=y_{0} \in$ $\mathcal{P}\left(L_{(0)}, L_{(1)}\right)$ (in a given connected component) to $v(1)=y$, which can also be viewed as map $v:[0,1] \times[0,1] \rightarrow \widetilde{M}$ satisfying Lagrangian boundary conditions on $\{0\} \times[0,1]$ and $\{1\} \times[0,1]$.

Suppose for now that $r$ is odd, then the path space can be identified with the set of tuples of paths in the manifolds $M_{j}$, connected via $L_{j(j+1)}$-matching conditions at the ends,

$$
\mathcal{P}(\underline{L})=\left\{\underline{x}=\left(x_{j}:[0,1] \rightarrow M_{j}\right)_{j=0, \ldots, r} \mid\left(x_{j}(1), x_{j+1}(0)\right) \in L_{j(j+1)}\right\} .
$$

Here and throughout we will use the index $j \in\{0, \ldots, r\}$ modulo $r+1$, so eg $x_{r+1}:=$ $x_{0}$ and the matching condition for $j=r+1$ is $\left(x_{r}(1), x_{0}(0)\right) \in L_{r(r+1)}$. We make the identification with $\mathcal{P}\left(L_{(0)}, L_{(1)}\right)$ by $y(t)=\left(x_{0}(1-t), x_{1}(t), x_{2}(1-t), \ldots, x_{r}(t)\right)$, then the unperturbed $(H=0)$ symplectic action functional on $\mathcal{P}(\underline{L})$ becomes

$$
\mathcal{A}_{0}(\underline{x})=\sum_{j=0}^{r} \int_{[0,1] \times[0,1]} v_{j}^{*} \omega_{M_{j}}
$$

Here $v_{j}:[0,1] \times[0,1] \rightarrow M_{j}$ interpolate between fixed paths $v_{j}(0, \cdot)$ and $v_{j}(1, \cdot)=x_{j}$, and satisfy what we will call "seam conditions" $\left(v_{j}(s, 1), v_{j+1}(s, 0)\right) \in L_{j(j+1)}$ for all $s \in[0,1]$. Next, assume that the almost complex structure on $\widetilde{M}$ is of time-independent split form $J=\left(-J_{0}\right) \oplus J_{1} \oplus\left(-J_{2}\right) \oplus \cdots \oplus J_{r}$, given by a tuple $J_{j} \in \mathcal{J}\left(M_{j}, \omega_{j}\right)$ of almost complex structures on the factors of $\widetilde{M}$. This defines a metric on the path space, and the gradient flow lines, viewed as solutions of PDE's are the $J$-holomorphic strips $w: \mathbb{R} \times[0,1] \rightarrow \widetilde{M}$ with boundary values in $L_{(0)}$ and $L_{(1)}$. They are in one-to-one correspondence with $(r+1)$-tuples of $J_{j}$-holomorphic maps $u_{j}: \mathbb{R} \times[0,1] \rightarrow M_{j}$ satisfying the seam conditions

$$
\left(u_{j}(s, 1), u_{j+1}(s, 0)\right) \in L_{j(j+1)}, \quad \text { for all } j=0, \ldots, r, s \in \mathbb{R} .
$$


Here we again use cyclic notation $u_{r+1}:=u_{0}$, and the correspondence is given by $w(s, t)=\left(u_{0}(s, 1-t), u_{1}(s, t), u_{2}(s, 1-t), \ldots, u_{r}(s, t)\right)$.

For $r$ even there is a slight modification of the previous correspondence. The product manifold $\widetilde{M}$ has two factors $M_{0}$ and $M_{r+1}=M_{0}$ matched up via the diagonal. So the path space can be identified with the generalized path space $\mathcal{P}(\underline{L})$ as above with the exception that the path $x_{0}:[0,2] \rightarrow M_{0}$ in $M_{0}=M_{r+1}$ is parametrized by an interval of length 2 and satisfies the matching condition $\left(x_{0}(2), x_{1}(0) \in L_{01}\right.$ at its end. Similarly, a $J$-holomorphic strip $w: \mathbb{R} \times[0,1] \rightarrow \widetilde{M}$ corresponds via

$$
w(s, t)=\left(u_{0}(s, 2-t), u_{1}(s, t), u_{2}(s, 1-t) \ldots, u_{r}(s, 1-t), u_{0}(s, t)\right)
$$

to a tuple of $J_{j}$-holomorphic strips as above, with the exception that the strip $u_{0}: \mathbb{R} \times$ $[0,2] \rightarrow M_{0}$ has width 2 . This tuple $\left(u_{j}\right)_{j=0, \ldots, r}$ is the first instance of a nontrivial pseudoholomorphic quilt - containing strips of different widths.

When $r$ is even, the Floer trajectories of the pair $L_{(0)}, L_{(1)}$ in fact cannot be identified with an $(r+1)$-tuple of pseudoholomorphic maps, all defined on strips of width 1 , with seam conditions in $L_{j(j+1)}$. Conformal rescaling $\tilde{u}_{0}(s, t):=u_{0}(2 s, 2 t)$ would result in a "time-shifted" matching condition $\left(\widetilde{u}_{0}(s, 1), u_{1}(2 s, 0)\right) \in L_{01}$ unless $u_{1}$ is rescaled, too, which would result in $\tilde{u}_{0}$ having width 1 but all other strips having width $\frac{1}{2}$. In fact, only simultaneous rescaling of all components in these pseudoholomorphic quilts preserves holomorphicity and seam conditions (unless the Lagrangian correspondences are of split type, eg $L_{01}=L_{0} \times L_{1}$ for Lagrangians $L_{j} \subset M_{j}$ ). It cannot change the relative widths of strips.

By a reparametrization of the path in $M_{0}$, one could identify $\mathcal{P}\left(L_{(0)}, L_{(1)}\right)$ and the action functional with the generalized path space $\mathcal{P}(\underline{L})$ and a corresponding action functional, where all paths are parametrized by $[0,1]$. However, the reparametrized $\widehat{u}_{0}(s, t):=u_{0}(s, 2 t)$ now satisfies $\partial_{s} \widehat{u}_{0}+\frac{1}{2} J_{0} \partial_{t} \widehat{u}_{0}=0$ with a no longer complex structure $\frac{1}{2} J_{0}$ that squares to $-\frac{1}{4}$. This is due to the fact that the pullback of the metric on $\mathcal{P}\left(L_{(0)}, L_{(1)}\right)$ to $\mathcal{P}(\underline{L})$ is the $L^{2}$-metric on each factor with respect to $\omega_{j}\left(\cdot, J_{j} \cdot\right)$ for $j=1, \ldots, r$ but $\frac{1}{2} \omega_{0}\left(\cdot, J_{0}\right)$ on $M_{0}$. We could drop the factor $\frac{1}{2}$ in the metric on $M_{0}$ to obtain $J_{j}$-holomorphic strips of width 1 in each factor as trajectories, however these would be the "gradient flow lines" with respect to a different metric. In general, it is not known how Floer homology behaves under a change of metric. However, we will show that it is independent of the choice of weights $\delta_{j}^{-1} \omega_{j}\left(\cdot, J_{j} \cdot\right)$ in the $L^{2}$-metric on $\mathcal{P}(\underline{L})$. This setup is equivalent to defining the generalized path space with varying widths $x_{j}:\left[0, \delta_{j}\right] \rightarrow M_{j}$ but fixing the standard $L^{2}$-metric induced by $\omega_{j}$ and $J_{j}$ on each factor. 


\subsection{Construction of quilted Floer cohomology}

In the quilted setup for $H F(\underline{L})$ we fix widths $\underline{\delta}=\left(\delta_{j}>0\right)_{j=0, \ldots, r}$ and consider the generalized path space

$$
\mathcal{P}(\underline{L}):=\left\{\underline{x}=\left(x_{j}:\left[0, \delta_{j}\right] \rightarrow M_{j}\right)_{j=0, \ldots, r} \mid\left(x_{j}\left(\delta_{j}\right), x_{j+1}(0)\right) \in L_{j(j+1)}\right\} .
$$

We define a perturbed symplectic action functional on $\mathcal{P}(\underline{L})$ by picking a homotopy $\underline{v}=\left(v_{j}\right)_{j=0, \ldots, r}:[0,1] \rightarrow \mathcal{P}(\underline{L})$ from a fixed $\underline{v}(0)$ to $\underline{v}(1)=\underline{x}$ and setting

$$
\mathcal{A}_{\underline{H}}(\underline{x})=\sum_{j=0}^{r}\left(\int_{[0,1] \times\left[0, \delta_{j}\right]} v_{j}^{*} \omega_{M_{j}}+\int_{0}^{\delta_{j}} H_{j}\left(t, x_{j}(t)\right) \mathrm{d} t\right),
$$

using a tuple of Hamiltonian functions

$$
\underline{H}=\left(H_{j} \in C^{\infty}\left(\left[0, \delta_{j}\right] \times M_{j}\right)\right)_{j=0, \ldots, r} .
$$

By folding and rescaling as in the previous section, this is equivalent to the path space $\mathcal{P}\left(L_{(0)}, L_{(1)}\right)$ with symplectic action functional perturbed by a Hamiltonian of split type, eg $H=\sum_{j=0}^{r}(-1)^{j+1} \delta_{j} \widetilde{H}_{j}$ for $r$ odd, where $\widetilde{H}_{j}(t, x)=H_{j}\left(\delta_{j} t, x\right)$ for $j$ odd and $\tilde{H}_{j}(t, x)=H_{j}\left(\delta_{j}(1-t), x\right)$ for $j$ even. Here the critical points correspond to the perturbed intersection points $\phi_{1}^{H}\left(L_{(0)}\right) \cap L_{(1)}$, where $\phi_{1}^{H}$ is the time-one flow of $H$. In the quilted setup, the critical points of $\mathcal{A}_{\underline{H}}$ are tuples of Hamiltonian chords

$$
\mathcal{I}(\underline{L}):=\left\{\begin{array}{l|r}
\underline{x}=\left(x_{j}:\left[0, \delta_{j}\right] \rightarrow M_{j}\right)_{j=0, \ldots, r} & \begin{array}{r}
\dot{x}_{j}(t)=X_{H_{j}}\left(x_{j}(t)\right), \\
\left(x_{j}\left(\delta_{j}\right), x_{j+1}(0)\right) \in L_{j(j+1)}
\end{array}
\end{array} .\right.
$$

$\mathcal{I}(\underline{L})$ is canonically identified with

$$
\times_{\phi_{\delta_{0}}}^{H_{0}}\left(L_{01} \times{ }_{\phi_{\delta_{1}}}^{H_{1}} L_{12} \cdots \times{ }_{\phi_{\delta_{r}}}^{H_{r}} L_{r(r+1)}\right),
$$

the set of points

$$
\left\{\left(m_{0}, \ldots, m_{r}\right) \in M_{0} \times \cdots \times M_{r} \mid\left(\phi_{\delta_{j}}^{H_{j}}\left(m_{j}\right), m_{j+1}\right) \in L_{j(j+1)}\right\},
$$

where $\phi_{\delta_{j}}^{H_{j}}$ is the time $\delta_{j}$ flow of the Hamiltonian $H_{j}$. In this setting we can check that Hamiltonians of split type suffice to achieve transversality for the intersection points.

Proposition 5.2.1 There is a dense open subset $\operatorname{Ham}(\underline{L}) \subset \bigoplus_{j=0}^{r} C^{\infty}\left(\left[0, \delta_{j}\right] \times M_{j}\right)$ such that for every $\left(H_{0}, \ldots, H_{r}\right) \in \operatorname{Ham}(\underline{L})$ the set

$$
\times{ }_{\delta_{\delta_{0}}}^{H_{0}}\left(L_{01} \times{ }_{\phi_{\delta_{1}}^{H_{1}}}^{H_{1}} L_{12} \cdots \times{ }_{\phi_{\delta_{r}}^{H_{r}}} L_{r(r+1)}\right)
$$

is smooth and finite, that is, the defining equations are transversal. 
Proof The defining equations for $\times_{\delta_{\delta_{0}}}^{H_{0}}\left(L_{01} \times \phi_{\delta_{1}}^{H_{1}} L_{12} \cdots \times{ }_{\phi_{\delta_{r}}}^{H_{r}} L_{r(r+1)}\right)$ are

$$
m_{j}^{\prime}=\phi_{\delta_{j}}^{H_{j}}\left(m_{j}\right) \quad \text { for all } j=0, \ldots, r
$$

for $\left(m_{0}^{\prime}, m_{1}, m_{1}^{\prime}, m_{2}, \ldots, m_{r}^{\prime}, m_{0}\right) \in L_{01} \times L_{12} \cdots \times L_{r(r+1)}$. Consider the universal moduli $\mathcal{U}$ space of data $\left(H_{0}, \ldots, H_{r}, m_{0}^{\prime}, m_{1}, \ldots, m_{r}^{\prime}, m_{0}\right)$ satisfying (25), where now each $H_{j}$ has class $C^{\ell}$ for some $\ell \geq 1$. It is cut out by the diagonal values of the $C^{\ell}$-map

$$
\begin{aligned}
L_{01} \times L_{12} \cdots \times L_{r(r+1)} \times \bigoplus_{j=0, \ldots, r} C^{\ell}\left([0,1] \times M_{j}\right) & \longrightarrow \bigoplus_{j=0, \ldots, r} M_{j} \times M_{j}, \\
& \left(m_{j}, m_{j}^{\prime}, H_{j}\right)_{j=0, \ldots, r} \longmapsto\left(\phi_{\delta_{j}}^{H_{j}}\left(m_{j}\right), m_{j}^{\prime}\right)_{j=0, \ldots, r} .
\end{aligned}
$$

The linearized equations for $\mathcal{U}$ are

$$
v_{j}^{\prime}-D \phi_{\delta_{j}}^{H_{j}}\left(h_{j}, v_{j}\right)=0 \in T M_{j} \quad \text { for all } j=0, \ldots, r,
$$

for $v_{j}, v_{j}^{\prime} \in T_{m_{j}} M_{j}$ and $h_{j} \in C^{\ell}\left([0,1] \times M_{j}\right)$. The map

$$
C^{\ell}\left([0,1] \times M_{j}\right) \rightarrow T_{\phi_{\delta_{j}}}^{H_{j}}\left(m_{j}\right) M_{j}, \quad h_{j} \mapsto D \phi_{\delta_{j}}^{H_{j}}\left(h_{j}, 0\right)
$$

is surjective, which shows that the product of the operators on the left-hand side of (26) is also surjective. So by the implicit function theorem $\mathcal{U}$ is a $C^{\ell}$ Banach manifold, and we consider its projection to $\bigoplus_{k=0}^{r} C^{\ell}\left(\left[0, \delta_{k}\right] \times M_{k}\right)$. This is a Fredholm map of class $C^{\ell}$ and index 0. Hence, by the Sard-Smale theorem, the set of regular values (which coincides with the set of functions $H=\left(H_{0}, \ldots, H_{r}\right)$ such that the perturbed intersection is transversal) is dense in $\bigoplus_{k=0}^{r} C^{\ell}\left(\left[0, \delta_{k}\right] \times M_{k}\right)$. Moreover, the set of regular values is open for each $\ell \geq 1$. Indeed, by the compactness of $L_{01} \times L_{12} \cdots \times L_{r(r+1)}$, a $C^{1}$-small change in $H$ leads to a small change in perturbed intersection points, with small change in the linearized operators.

Now, by approximation of $C^{\infty}$-functions with $C^{\ell}$-functions, the set of regular values in $\bigoplus_{k=0}^{r} C^{\infty}\left(\left[0, \delta_{k}\right] \times M_{k}\right)$ is dense in the $C^{\ell}$-topology for all $\ell \geq 1$, and hence dense in the $C^{\infty}$-topology. Finally, the set of regular smooth $H$ is open in the $C^{\infty}$-topology as a special case of the $C^{1}$-openness.

In the proof of Theorem 5.4.1 we will use the following special choice of corresponding regular Hamiltonian perturbations, which will provide a canonical identification of intersection points, as in Remark 3.1.3 for the unperturbed case.

Remark 5.2.2 Consider two cyclic generalized Lagrangian correspondences

$$
\begin{aligned}
\underline{L} & =\left(L_{01}, \ldots, L_{(j-1) j}, L_{j(j+1)}, \ldots, L_{r(r+1)}\right), \\
\underline{L}^{\prime} & =\left(L_{01}, \ldots, L_{(j-1) j} \circ L_{j(j+1)}, \ldots, L_{r(r+1)}\right)
\end{aligned}
$$


such that the composition $L_{(j-1) j} \circ L_{j(j+1)}$ is embedded in the sense of Definition 2.0.4. Pick Hamiltonian perturbations $\underline{H}^{\prime}=\left(\ldots, H_{j-1}, H_{j+1}, \ldots\right) \in \operatorname{Ham}\left(\underline{L}^{\prime}\right)$ such that

$$
\times_{\phi_{\delta_{0}}}^{H_{0}}\left(L_{01} \cdots \times_{\phi_{\delta_{j-1}}^{H_{j-1}}}^{H_{(j-1) j}} \circ L_{j(j+1)} \times_{\left.\phi_{\delta_{j+1}}^{H_{j+1}} \cdots L_{r(r+1)}\right)}\right.
$$

is transverse. Then we have $\underline{H}:=\left(\ldots, H_{j-1}, H_{j} \equiv 0, H_{j+1}, \ldots\right) \in \operatorname{Ham}(\underline{L})$, that is

$$
\times_{\phi_{\delta_{0}}}^{H_{0}}\left(L_{01} \cdots \times_{\phi_{\delta_{j-1}}^{H_{j-1}}}^{H_{j-1) j}} L_{\mathrm{Id}} L_{j(j+1)} \times_{\phi_{\delta_{j+1}}}^{H_{j+1}} \cdots L_{r(r+1)}\right)
$$

is transverse, since by assumption $L_{(j-1) j} \times L_{(j+1) j}$ is transverse to the diagonal $M_{j-1} \times \Delta_{M_{j}} \times M_{j+1}$. Moreover, the generalized intersection points

$$
\begin{aligned}
\mathcal{I}(\underline{L}, \underline{H})=\left\{\left(\ldots, m_{j-1}, m_{j}, m_{j+1}, \ldots\right) \in \cdots \times M_{j-1} \times M_{j} \times M_{j+1} \cdots \mid\right. \\
\left.\ldots,\left(\phi_{\delta_{j-1}}^{H_{j-1}}\left(m_{j-1}\right), m_{j}\right) \in L_{(j-1) j},\left(m_{j}, m_{j+1}\right) \in L_{j(j+1)}, \ldots\right\} \\
=\left\{\left(\ldots, m_{j-1}, m_{j+1}, \ldots\right) \in \cdots \times M_{j-1} \times M_{j+1} \cdots \mid\right. \\
\left.\quad \ldots,\left(\phi_{\delta_{j-1}}^{H_{j-1}}\left(m_{j-1}\right), m_{j+1}\right) \in L_{(j-1) j} \circ L_{j(j+1)}, \ldots\right\}=\mathcal{I}\left(\underline{L}^{\prime}, \underline{H^{\prime}}\right)
\end{aligned}
$$

are canonically identified, since the intermediate point $m_{j} \in M_{j}$ is uniquely determined by the pair

$$
\left(\phi_{\delta_{j-1}}^{H_{j-1}}\left(m_{j-1}\right), m_{j+1}\right) \in L_{(j-1) j} \circ L_{j(j+1)} .
$$

With this split Hamiltonian perturbation we have a canonical bijection of critical points $\phi_{1}^{H}\left(L_{(0)}\right) \cap L_{(1)} \cong \mathcal{I}(\underline{L})$, and hence the (graded) Floer chain group $C F\left(L_{(0)}, L_{(1)}\right)$ is identified with

$$
C F(\underline{L}):=\bigoplus_{d \in \mathbb{Z}_{N}} C F^{d}(\underline{L}), \quad C F^{d}(\underline{L}):=\bigoplus_{\underline{x} \in \mathcal{I}(\underline{L}),|\underline{x}|=d} \mathbb{Z}\langle\underline{x}\rangle .
$$

The grading is defined as in Section 3.1,

$$
\mathcal{I}(\underline{L}) \cong \phi_{1}^{H}\left(L_{(0)}\right) \cap L_{(1)} \rightarrow \mathbb{Z}_{N}, \quad \underline{x} \cong y \mapsto|y|=|\underline{x}| .
$$

Next, fix a tuple of almost complex structures

$$
\underline{J}=\left(J_{j}\right)_{j=0, \ldots, r} \in \bigoplus_{j=0}^{r} C^{\infty}\left(\left[0, \delta_{j}\right], \mathcal{J}\left(M_{j}, \omega_{j}\right)\right)=: \mathcal{J}_{t}(\underline{L})
$$

and equip $\mathcal{P}(\underline{L})$ with the $L^{2}$-metric induced by the $t$-dependent metric $\omega_{j}\left(\cdot, J_{j} \cdot\right)$ on each factor $M_{j}$. Then the Floer trajectories (obtained by reformulating the gradient flow as PDE) are $(r+1)$-tuples of maps $u_{j}: \mathbb{R} \times\left[0, \delta_{j}\right] \rightarrow M_{j}$ that are $\left(J_{j}, H_{j}\right)-$ holomorphic,

$$
\bar{\partial}_{J_{j}, H_{j}} u_{j}=\partial_{s} u_{j}+J_{j}\left(\partial_{t} u_{j}-X_{H_{j}}\left(u_{j}\right)\right)=0 \quad \forall j=0, \ldots r,
$$


and satisfy the seam conditions

$$
\left(u_{j}\left(s, \delta_{j}\right), u_{j+1}(s, 0)\right) \in L_{j(j+1)} \quad \forall j=0, \ldots r, s \in \mathbb{R} .
$$

For a Floer trajectory to be counted towards the differential between critical points $\underline{x}^{ \pm} \in \mathcal{I}(\underline{L})$ we moreover require finite energy and limits

$$
\begin{gathered}
E(\underline{u}):=\sum_{j=0}^{r} \int_{\mathbb{R} \times\left[0, \delta_{j}\right]} u_{j}^{*} \omega_{j}+\mathrm{d}\left(H_{j}\left(u_{j}\right) \mathrm{d} t\right)<\infty, \\
\lim _{s \rightarrow \pm \infty} u_{j}(s, \cdot)=x_{j}^{ \pm},
\end{gathered}
$$$$
\forall j=0, \ldots, r \text {. }
$$

As in standard Floer theory, the moduli spaces of "quilted holomorphic strips"
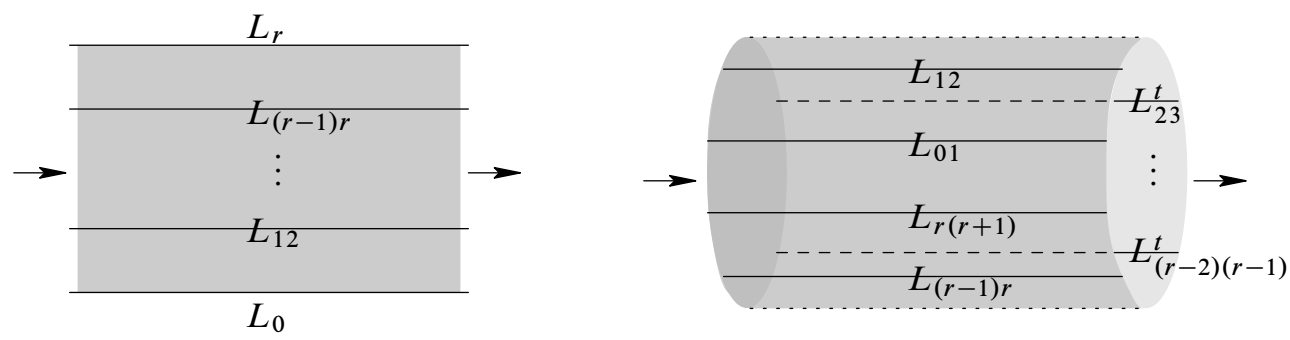

Figure 1: Quilted Floer trajectories for $M_{0}=\{\mathrm{pt}\}$ and in general

$$
\mathcal{M}\left(\underline{x}^{-}, \underline{x}^{+}\right):=\left\{\underline{u}=\left(u_{j}: \mathbb{R} \times\left[0, \delta_{j}\right] \rightarrow M_{j}\right)_{j=0, \ldots, r} \mid(27),(28),(29)\right\} / \mathbb{R}
$$

arise from quotienting out by simultaneous $\mathbb{R}$-shift in all components $u_{j}$. (Separate shifts will not preserve the seam condition unless the correspondences are of split type.) We will see that they have the same Fredholm, exponential decay, and compactness properties as usual for Floer trajectories. For that purpose we restrict ourselves to the monotone case.

Remark 5.2.3 The "monotonicity for Floer theory" assumption for the pair $\left(L_{(0)}, L_{(1)}\right)$ in Definition 4.1 .2 can be phrased directly for $\underline{L}$ in the language of [41]: " $\underline{L}$ is a monotone boundary condition for the quilted cylinder". That is, the action-index relation

$$
2 \sum_{j=0}^{r} \int u_{j}^{*} \omega_{j}=\tau \cdot I\left(\left(u_{j}^{*} T M_{j}\right)_{j=0, \ldots, r},\left(s_{j(j+1)}^{*} T L_{j(j+1)}\right)_{j=0, \ldots, r}\right)
$$

holds for each tuple of maps $u_{j}: S^{1} \times\left[0, \delta_{j}\right] \rightarrow M_{j}$ that satisfies the seam conditions $s_{j(j+1)}(s):=\left(u_{j}\left(s, \delta_{j}\right), u_{j+1}(s, 0)\right) \in L_{j(j+1)}$ for $j=0, \ldots, r$. Here the topological 
index $I$ is defined by choosing a trivialization for each $u_{j}^{*} T M_{j}$ and then summing over the Maslov indices of the loops $s_{j(j+1)}^{*} T L_{j(j+1)}$ of Lagrangian subspaces with respect to these trivializations.

Note that the monotonicity condition for $\underline{L}$ is independent of the width $\delta_{j}$ of the annuli that parametrize the maps $u_{j}$. Moreover, it implies monotonicity for the sequence $\underline{L}^{\prime}=\left(L_{01}, \ldots, L_{(j-1) j} \circ L_{j(j+1)}, \ldots, L_{r(r+1)}\right)$ obtained from an embedded composition $L_{(j-1) j} \circ L_{j(j+1)}$. To see the latter note that any seam condition $s_{(j-1)(j+1)}: S^{1} \rightarrow L_{(j-1) j} \circ L_{j(j+1)}$ induces a smooth map $u_{j}: S^{1} \times[0,1] \rightarrow M_{j}$ that is constant in $[0,1]$, fits the seam conditions for $\underline{L}$, but contributes zero to both energy and Maslov index. Hence the action index relation for $\underline{L}$ implies the same relation for $\underline{L}^{\prime}$. Indeed, to identify the Maslov indices pick the trivialization of $u_{j}^{*} T M_{j}$ constant across $[0,1]$. Then the Maslov index for $\underline{L}$ has a contribution $I\left(\Lambda_{(j-1) j}\right)+I\left(\Lambda_{j(j+1)}\right)=I\left(\Lambda_{(j-1) j} \times \Lambda_{j(j+1)}\right)$ from the trivializations $\Lambda_{i(i+1)}: S^{1} \rightarrow \mathbb{C}^{n_{i}+n_{i+1}}$ of $s_{i(i+1)}^{*} L_{i(i+1)}$, and the contribution to the Maslov index for $\underline{L}^{\prime}$ is the index $I\left(\Lambda_{(j-1)(j+1)}:=\Lambda_{(j-1) j} \circ \Lambda_{j(j+1)}\right)$ of the geometric composition of the trivializations. By Lemma 3.1.9 we can homotope $\Lambda_{(j-1) j} \times \Lambda_{j(j+1)}$ to $\left(\Lambda_{(j-1)(j+1)} \times \Lambda_{11}\right)^{T}$, where $\Lambda_{11} \subset \mathbb{C}^{n_{1}} \times \mathbb{C}^{n_{1}}$ is a fixed complement of the diagonal and $(\cdot)^{T}$ is the exchange of factors as in Section 3.1. Then the crossing form definition of the Maslov index proves

$$
\begin{aligned}
I\left(\Lambda_{(j-1) j} \times \Lambda_{j(j+1)}\right) & =I\left(\left(\Lambda_{(j-1)(j+1)} \times \Lambda_{11}\right)^{T}\right) \\
& =I\left(\Lambda_{(j-1)(j+1)}\right)+I\left(\Lambda_{11}\right)=I\left(\Lambda_{(j-1)(j+1)}\right) .
\end{aligned}
$$

Theorem 5.2.4 Suppose that the symplectic manifolds $M_{j}$ satisfy(M1)-(M2) with the same value of the monotonicity constant $\tau$, the Lagrangian correspondences $L_{j(j+1)}$ satisfy (L1)-(L2), and $\underline{L}$ satisfies the monotonicity assumption of Remark 5.2.3.

For any choice of widths $\underline{\delta}$ and regular Hamiltonian perturbations $\underline{H} \subset \operatorname{Ham}(\underline{L})$ there exists a dense subset $\mathcal{J}_{t}^{\text {reg }}(\underline{L} ; \underline{H}) \subset \mathcal{J}_{t}(\underline{L})$ such that the following holds for all $\underline{x}_{ \pm} \in \mathcal{I}(\underline{L})$.

(a) $\mathcal{M}\left(\underline{x}_{-}, \underline{x}_{+}\right)$is a smooth manifold whose dimension near a nonconstant solution $\underline{u}$ is given by the formal dimension, equal to $\operatorname{Ind}\left(D_{\underline{u}}\right)-1$. Here $D_{\underline{u}}$ is the linearized operator at $\underline{u}$ of (27) on the space of sections satisfying the linearized boundary- and seam conditions of (28). We denote the component of nonconstant solutions of formal dimension $j$ by $\mathcal{M}\left(\underline{x}_{-}, \underline{x}_{+}\right)_{j}:=\left\{\operatorname{Ind}\left(D_{\underline{u}}\right)-1=j\right\}$.

(b) The component $\mathcal{M}\left(\underline{x}_{-}, \underline{x}_{+}\right)_{0} \subset \mathcal{M}\left(\underline{x}_{-}, \underline{x}_{+}\right)$of formal dimension zero is finite.

(c) Suppose that each $L_{j(j+1)}$ has minimal Maslov number $N_{L_{j(j+1)}} \geq 3$. Then the one-dimensional component $\mathcal{M}\left(\underline{x}_{-}, \underline{x}_{+}\right){ }_{1} \subset \mathcal{M}\left(\underline{x}_{-}, \underline{x}_{+}\right)$has a compactification 
as one-dimensional manifold with boundary

$$
\partial \overline{\mathcal{M}\left(\underline{x}_{-}, \underline{x}_{+}\right)_{1}} \cong \bigcup_{\underline{x} \in \mathcal{I}(\underline{L})} \mathcal{M}\left(\underline{x}_{-}, \underline{x}\right)_{0} \times \mathcal{M}\left(\underline{x}, \underline{x}_{+}\right)_{0} .
$$

(d) If $\underline{L}$ is relatively spin (as defined in Definition 4.3.1), then there exists a coherent set of orientations on $\mathcal{M}\left(\underline{x}_{-}, \underline{x}_{+}\right)_{0}, \mathcal{M}\left(\underline{x}_{-}, \underline{x}_{+}\right)_{1}$ for all $\underline{x}_{ \pm} \in \mathcal{I}(\underline{L})$, that is, orientations compatible with (30).

Proof Suppose for simplicity that $r$ is odd. (For even $r$ we can insert a diagonal into the sequence $\underline{L}$, then the quilted holomorphic strips of widths $\underline{\delta}$ can be identified with quilted holomorphic strips for the new sequence with widths $\left(\frac{\delta_{0}}{2}, \delta_{1}, \ldots, \delta_{r}, \frac{\delta_{0}}{2}\right)$.) Then the quilted moduli space $\mathcal{M}\left(\underline{x}_{-}, \underline{x}_{+}\right)$is canonically identified with the moduli space of $\left(J_{\underline{\delta}}, H\right)$-holomorphic maps $w: \mathbb{R} \times[0,1] \rightarrow \widetilde{M}$ with boundary conditions $w(\mathbb{R}, 0) \subset \bar{L}_{(0)}, w(\mathbb{R}, 1) \subset L_{(1)}$, finite energy $E_{H}(w)<\infty$, and limit conditions $\lim _{s \rightarrow \pm \infty} w(s, \cdot)=\underline{x}_{ \pm} \in \mathcal{I}\left(L_{(0)}, L_{(1)}\right)$.

The correspondence is by

$$
w(s, t)=\left(u_{0}\left(s, \delta_{0}(1-t)\right), u_{1}\left(s, \delta_{1} t\right), u_{2}\left(s, \delta_{2}(1-t)\right), \ldots, u_{r}\left(s, \delta_{r} t\right)\right),
$$

where $H=\sum_{j=0}^{r}(-1)^{j+1} \delta_{j} \widetilde{H}_{j}$ as above and

$$
J_{\underline{\delta}}:=\left(-\delta_{0}^{-1} J_{0}\left(\delta_{0}(1-t)\right), \delta_{1}^{-1} J_{1}\left(\delta_{1} t\right), \ldots, \delta_{r}^{-1} J_{r}\left(\delta_{r} t\right)\right)
$$

satisfies all properties of a $t$-dependent $\omega_{\widetilde{M}}$-compatible almost complex structure except that it squares to the negative definite diagonal matrix

$$
J_{\underline{\delta}}^{2}=-\left(\delta_{0}^{-2} \operatorname{Id}_{T M_{0}} \oplus \ldots \oplus \delta_{r}^{-2} \operatorname{Id}_{T M_{r}}\right)
$$

instead of -Id. Let us call it a "scaled almost complex structure". Most analytic properties of pseudo-holomorphic curves carry over directly to $J_{\underline{\delta}}$-holomorphic curves. Indeed, $\bar{\partial}_{J_{\underline{\delta}}, H}$ still presents a partial differential operator of the form $\partial_{s}+\mathcal{D}$, where the linearizations of $\mathcal{D}$ are self-adjoint operators on $L^{2}([0,1], T \widetilde{M})$ with boundary conditions in $T L_{(0)}, T L_{(1)}$. Moreover, in local coordinates $\left(\partial_{s}-\mathcal{D}\right)\left(\partial_{s}+\mathcal{D}\right)=$ $\partial_{s}^{2}-J_{\delta}^{2} \partial_{t}^{2}+$ (lower order terms) is an elliptic operator (ie has an elliptic symbol), and in the splitting $T \widetilde{M}=T L_{(0)} \oplus J_{\underline{\delta}}^{-1} T L_{(0)}$ the Lagrangian boundary conditions induce Neumann conditions (from $\left.\partial_{t} w^{\underline{\delta}} X_{H}(w)\right|_{t=0}=-\left.J_{\underline{\delta}}^{-1} \partial_{s} w\right|_{t=0} \in J_{\underline{\delta}}^{-1} T L_{(0)}$ ) resp. Dirichlet conditions (from $\left.w\right|_{t=0} \in L_{(0)}$ ) on the two factors.

With these remarks in mind, we can follow the standard construction of Floer theory (which is currently probably best outlined by Salamon [29] for the case of holomorphic cylinders, fully executed by Donaldson [5] for a gauge theoretic setting, and hopefully soon available in Oh [20] for holomorphic strips). The moduli space (before quotienting 
by the $\mathbb{R}$-action) is described as the zero set of the scaled Cauchy-Riemann operator $\bar{\partial}_{J_{\underline{\delta}}, H}$, which is a Fredholm section of a Banach bundle over the usual Banach manifold of maps $w: \mathbb{R} \times[0,1] \rightarrow \widetilde{M}$ satisfying boundary conditions in $L_{(0)}, L_{(1)}$ and converging uniformly to $\underline{x}_{ \pm}$for $s \rightarrow \pm \infty$. (The Banach manifold is modeled as usual for some $p>2$ on the Sobolev space of maps in $W^{1, p}\left(\mathbb{R} \times[0,1], \mathbb{C}^{N}\right)$ which take boundary values in $\mathbb{R}^{N}$ resp. $i \mathbb{R}^{N}$.)

The Fredholm property of the linearized operators follows as in eg Floer [8] from the general theory of Lockhart and McOwen [17] (also see Donaldson [5]) for operators of the form $\partial_{s}+D_{s}$, where the operators $D_{s}$ converge to invertible operators as $s \rightarrow \pm \infty$. After a Hamiltonian transformation (moving the perturbation onto the Lagrangian $L_{(0)}$ and replacing $J_{\underline{\delta}}$ with $\phi_{*}^{H} J_{\underline{\delta}}$, which retains the same properties) these operators take the form $\left(\phi_{*}^{\bar{H}} J_{\underline{\delta}}\right) \partial_{t}$ on $L^{p}([0,1], T \widetilde{M})$ with domain given by $W^{1, p}$-paths satisfying boundary conditions in $T \phi_{1}^{H}\left(L_{(0)}\right), T L_{(1)}$. Note that $\phi_{*}^{H} J_{\underline{\delta}}$ is an invertible operator on $L^{p}([0,1], T \widetilde{M})$, and invertibility of $\partial_{t}$ follows as usual from the fact that the boundary conditions are transverse on the ends $s \rightarrow \pm \infty$. Similar considerations (for $p=2$ showing that each $D_{s}$ is self-adjoint and for sufficiently large $|s|$ has a spectral gap - eigenvalues bounded uniformly away from 0 ) are the crucial ingredient in proving that solutions of the nonlinear equation on long strips of small energy converge exponentially to intersection points. Details can be found in Floer [8], Salamon [29] or our paper [38, Lemma 3.2.3] (where we prove the presently irrelevant fact that the exponential decay rate is in fact uniform for certain families of widths $\underline{\delta}$ ).

To calculate the index of the linearization of $\bar{\partial}_{J_{\underline{\delta}}, H}$, one can deform $J_{\underline{\delta}}$ through the endomorphisms $\left(\exp \left(\tau \ln \delta_{0}\right) \operatorname{Id}_{T M_{0}}, \ldots, \exp \left(\tau \ln \delta_{r}\right) \operatorname{Id}_{T M_{r}}\right) \circ J_{\underline{\delta}}$ to a true almost complex structure at $\tau=1$. This provides a continuous family of Fredholm operators, along which the index is constant, and ending at a traditional Cauchy-Riemann operator whose index is given by a Maslov index. (see eg Floer [7] or Seidel [33]). In particular, we have $\operatorname{ind}\left(D_{\underline{u}}\right)=0$ for the constant solution in case $\underline{x}_{-}=\underline{x}_{+}$. This identification of the index with a Maslov index together with the monotonicity assumption implies an energy-index relation as in Remark 4.2.2 for solutions (for another proof see eg Oh [22]). Hence all solutions with a fixed index satisfy a uniform $L^{2}$-bound on the gradient. But before proceeding to compactness, let us assume that the section $\bar{\partial}_{\delta_{\underline{\delta}}, H}$ is transverse to the zero section, ie its linearization at any zero is surjective. (This will be achieved by an appropriate choice of $\underline{J}$ - see below.) Then the implicit function theorem for Banach bundles (see eg McDuff and Salamon [18]) implies that the space of solutions $\left(\mathcal{M}\left(\underline{x}_{-}, \underline{x}_{+}\right)\right.$before quotienting by $\left.\mathbb{R}\right)$ is a smooth manifold, whose dimension near $\underline{u}$ is the Fredholm index $\operatorname{ind}\left(D_{\underline{u}}\right)$. Now, except for at a constant solution, the $\mathbb{R}$-action on these finite dimensional manifolds is smooth, proper, and 
free (whereas on the Banach manifold it is not even differentiable), inducing a smooth structure on the moduli spaces $\mathcal{M}\left(\underline{x}_{-}, \underline{x}_{+}\right)$of dimension ind $\left(D_{\underline{u}}\right)-1$ at nonconstant solutions. This proves (a).

To prove (b) and (c) one next proves compactness properties of the moduli spaces of fixed index, as in Floer [8; 29]. By monotonicity, the fixed index provides a uniform $L^{2}$-bound on the gradients of solutions. If, on the other hand, one had an $L^{\infty}$ gradient bound for a sequence of solutions, then elliptic estimates would imply that a subsequence converges with all derivatives on all compact subsets of $\mathbb{R} \times[0,1]$. (These estimates work exactly as in [18, Appendix B], using the splitting into Dirichlet and Neumann problem described above.) For the moduli spaces of index 1 and 2 we can ensure $L^{\infty}$ bounds as follows: We analyze any blow-up point of the gradient in the formulation as a tuple of maps $\underline{u}$. Then the usual rescaling analysis (see eg Floer [8]) is local, in the interior of one component $u_{j}$ (leading to a $J_{j}$-holomorphic sphere in $M_{j}$ ) or near a seam, where we can consider $u_{j}(s,-t) \times u_{j+1}(s, t)$ as $\left(-J_{j}\right) \oplus J_{j+1}$-holomorphic curve with boundary condition in $L_{j(j+1)}$. The latter type of bubbling hence leads to a holomorphic disc in $M_{j}^{-} \times M_{j+1}$ with boundary on $L_{j(j+1)}$. Away from these blow-up points, the solution converges $C_{\mathrm{loc}}^{\infty}$ to a punctured solution, to which we can apply the usual removable singularity theorems (in eg McDuff and Salamon [18] and Oh [21]). In the limit we obtain a new solution (possibly with different end points) of nonnegative index and a number of holomorphic spheres and disks, each of which is nonconstant, so by monotonicity has positive index. By assumption (L3) they in fact must have Maslov index at least 3 (ie Chern number 2 for spheres). Since the Maslov index of all components adds up to 1 or 2, any bubbling is excluded. (See Oh [22] for the analogous argument in the standard theory.) This discussion ensures that the moduli spaces of index 1 and 2 are compact up to the breaking of trajectories as in Morse theory. This is proven by combining the local elliptic estimates with the exponential decay on long strips; see Floer [8] and Donaldson [5]. Finally, part (c) requires a gluing theorem identifying the ends of the moduli space with broken trajectories. Again the proof in Floer [6] (or Schwarz [30, Section 3.2] for the closed case) carries over directly. The crucial ingredient is a uniformly bounded family of right inverses for the linearized operator as in Salamon [29, Proposition 3.9], which is established by combining the already established exponential decay and Fredholm estimates.

Orientations can also be defined as in the standard Floer theory [33] since the linearized operator canonically deforms (as above) through Fredholm operators to a standard Cauchy-Riemann operator.

The only part of the standard construction of Floer theory on $\widetilde{M}$ that has to be adapted substantially is transversality: The scaled almost complex structures $J_{\delta}$ obtained from tuples $\underline{J}$ of almost complex structures on each factor of $\widetilde{M}$ are highly nongeneric as 
scaled almost complex structures on $\widetilde{M}$ (which generically do not respect the splitting into factors). Nevertheless, we proceed as usual and define the set $\mathcal{J}_{t}^{\text {reg }}(\underline{L} ; \underline{H})$ to be those tuples of complex structures $\underline{J}$, such that the corresponding scaled almost complex structure $J_{\underline{\delta}} \in \mathcal{J}_{t}\left(L_{(0)}, L_{(1)} ; H\right)$ is regular in the sense that the linearized operator $D_{u} \bar{\partial}_{J_{\underline{\delta}}, H}$ is surjective for every solution of $\bar{\partial}_{J_{\underline{\underline{\delta}}}, H} u=0$ with $L_{(0)}, L_{(1)}$ boundary values. (This is equivalent to the surjectivity at every solution $\underline{u}$ of the linearized operator $D_{\underline{u}}$ of (27) on the space of sections satisfying the linearized boundary- and seam conditions of (28).) In order to find a dense set of regular $\underline{J}$ we note that the unique continuation theorem [9, Theorem 4.3] applies to the interior of every single nonconstant strip $u_{j}: \mathbb{R} \times\left(0, \delta_{j}\right) \rightarrow M_{j}$. It implies that the set of regular points, $\left(s_{0}, t_{0}\right) \in \mathbb{R} \times\left(0, \delta_{j}\right)$ with $\partial_{s} u_{j}\left(s_{0}, t_{0}\right) \neq 0$ and $u_{j}^{-1}\left(u_{j}(\mathbb{R} \cup\{ \pm \infty\}), t_{0}\right)=\left\{\left(s_{0}, t_{0}\right)\right\}$, is open and dense. These points can be used to prove surjectivity of the linearized operator for a universal moduli space of solutions with respect to almost complex structures of class $C^{k}$. (The constant solutions are automatically transverse due to the previously ensured transversality of the intersection points $\phi_{1}^{H}\left(L_{(0)}\right) \pitchfork L_{(1)}$.) The existence of a $C^{k}$-dense set of regular $\underline{J}$ then follows from the usual Sard-Smale argument as in [18] for each $k \in \mathbb{N}$. Finally, an intersection argument by Taubes (which here works exactly as in [9]) proves that the regular smooth almost complex structures are a comeagre ${ }^{7}$ subset of $\mathcal{J}_{t}(\underline{L})$ in the $C^{\infty}$-topology. Since $\mathcal{J}_{t}(\underline{L})$ is a complete metric space ${ }^{8}$, and hence a Baire space, any such comeagre subset is dense as claimed; see eg Royden [28, Theorem 27].

Now, assuming monotonicity and choosing regular $\underline{H}$ and $\underline{J}$ we can define the Floer homology $H F(\underline{L})$ just as in the standard case: The Floer coboundary operator $\partial^{d}: C F^{d}(\underline{L}) \rightarrow C F^{d+1}(\underline{L})$ is defined by

$$
\partial^{d}\left\langle\underline{x}_{-}\right\rangle:=\sum_{\underline{x}_{+} \in \mathcal{I}(\underline{L})}\left(\sum_{\underline{u} \in \mathcal{M}\left(\underline{x}_{-}, \underline{x}_{+}\right)_{0}} \epsilon(\underline{u})\right)\left\langle\underline{x}_{+}\right\rangle,
$$

where the signs $\epsilon: \mathcal{M}\left(\underline{x}_{-}, \underline{x}_{+}\right)_{0} \rightarrow\{ \pm 1\}$ are defined by comparing the given orientation to the canonical orientation of a point. It follows from Theorem 5.2.4 (c) that $\partial^{2}=0$, and $\partial$ is a map of degree 1 by index calculations as in the standard case. This defines

${ }^{7}$ A subset of a topological space is comeagre if it is the intersection of countably many open dense subsets. Many authors in symplectic topology would use the term "Baire second category", which however in classical Baire theory [28, Chapter 7.8] denotes more generally subsets that are not meagre, ie not the complement of a comeagre subset.

${ }^{8}$ It can be expressed as a closed subspace of a function space $C^{\infty}\left([0,1] \times \widetilde{M}, \mathbb{R}^{N}\right)$. The latter carries a metric $d(f, g)=\sum_{k=0}^{\infty} 2^{-k}\left\|\nabla^{k}(f-g)\right\|_{\infty}\left(1+\left\|\nabla^{k}(f-g)\right\|_{\infty}\right)^{-1}$, which induces the $C^{\infty}$-topology. 
the quilted Floer cohomology

$$
H F(\underline{L}):=\bigoplus_{d \in \mathbb{Z}_{N}} H F^{d}(\underline{L}), \quad H F^{d}(\underline{L}):=\operatorname{ker}\left(\partial^{d}\right) / \operatorname{im}\left(\partial^{d-1}\right)
$$

as $\mathbb{Z}_{N}$-graded group. It is independent of the choice of $\underline{H}$ and $\underline{J}$ by a standard construction of continuation maps. The same construction also allows for a deformation of the widths $\underline{\delta}$, in the folded setup of the above proof, where the $\delta_{j}$ are merely scale factors in the endomorphism $J_{\delta}$. For a more conceptual proof based on quilts interpolating between strips of different widths see Section 5.3 below.

Remark 5.2.5 One can also allow the sequence $\underline{L}$ to have length zero (that is, the empty sequence) as a generalized correspondence from $M$ to $M$; this is the case $r=-1$ in the previous notation. In this case we define $H F(\underline{L})=H F\left(\operatorname{Id}_{M}\right)$, the cylindrical Floer homology. This would be the case without seams in Figure 1.

In the special case of a cyclic generalized Lagrangian correspondence $\underline{L}$ with $M_{0}=\{\mathrm{pt}\}$ and some intermediate correspondence $L_{j(j+1)}$ of split form, we have the following Künneth Theorem.

Theorem 5.2.6 Suppose that $\underline{L}=\left(L_{01}, \ldots, L_{r(r+1)}\right)$ is a cyclic generalized Lagrangian correspondence as in Theorem 5.2.4 such that $M_{0}=\{\mathrm{pt}\}$ and $L_{j(j+1)}=$ $L_{j} \times L_{j+1}$ for some $1 \leq j<r$ and Lagrangian submanifolds $L_{j} \subset M_{j}$ and $L_{j+1} \subset$ $M_{j+1}$. Then the quilted Floer complex for $\underline{L}$ is canonically isomorphic to the tensor product of the two Floer complexes for the cyclic correspondences $\left(L_{01}, \ldots, L_{j}\right)$ and $\left(L_{j+1}, \ldots, L_{r(r+1)}\right)$,

$C F\left(L_{01}, \ldots, L_{j} \times L_{j+1}, \ldots, L_{r(r+1)}\right)$

$$
\cong C F\left(L_{01}, \ldots, L_{j}\right) \otimes C F\left(L_{j+1}, \ldots, L_{r(r+1)}\right) .
$$

In particular, if either $H F\left(L_{01}, \ldots, L_{j}\right)$ or $H F\left(L_{j+1}, \ldots, L_{r(r+1)}\right)$ is torsion-free, then there is a canonical isomorphism

$H F\left(L_{01}, \ldots, L_{j} \times L_{j+1}, \ldots, L_{r(r+1)}\right)$

$$
\cong H F\left(L_{01}, \ldots, L_{j}\right) \otimes H F\left(L_{j+1}, \ldots, L_{r(r+1)}\right) .
$$

The latter isomorphism also holds if we work with coefficients in a field, eg with $\mathbb{Z}_{2}$ coefficients.

Proof The generators and boundary operators of the Floer complex $C F\left(L_{01}, \ldots\right.$, $\left.L_{j} \times L_{j+1}, \ldots, L_{r(r+1)}\right)$ are trivially identified with the generators and differential of 
the Floer complex $C F\left(L_{01}, \ldots, L_{j}, L_{j+1}, \ldots, L_{r(r+1)}\right)$, viewing the two factors of $L_{j} \times L_{j+1}$ as correspondences $L_{j} \subset M_{j}^{-} \times\{\mathrm{pt}\}$ and $L_{j+1} \subset\{\mathrm{pt}\}^{-} \times M_{j+1}$ to and from the point. This is the first trivial case of Theorem 5.4.1 below. At the same time, the intersection points of $\underline{L}$ are trivially identified with the pairs of intersection points of $\left(L_{01}, \ldots, L_{j}\right)$ and $\left(L_{j+1}, \ldots, L_{r(r+1)}\right)$, and the Floer trajectories for $\underline{L}$ are either a pair of a Floer trajectory for $\left(L_{01}, \ldots, L_{j}\right)$ and an intersection point of $\left(L_{j+1}, \ldots, L_{r(r+1)}\right)$ or a pair of an intersection point for $\left(L_{01}, \ldots, L_{j}\right)$ and a Floer trajectory for $\left(L_{j+1}, \ldots, L_{r(r+1)}\right)$. This canonically identifies the Floer complex $C F(\underline{L})$ with the tensor product of complexes $C F\left(L_{01}, \ldots, L_{j}\right) \otimes C F\left(L_{j+1}, \ldots, L_{r(r+1)}\right)$.

Now the isomorphism of homologies follows from the general Künneth formula [35, Lemma 5.3.1] and the fact that the torsion product vanishes if it has one torsion-free factor [35, Lemma 5.2.5]. Modules over fields are generally torsion-free.

\subsection{Invariance of quilted Floer cohomology and relative quilt invariants}

The purpose of this section is to prove the independence of quilted Floer cohomology from the choice of perturbation data, in particular the choice of widths.

Consider a cyclic generalized Lagrangian correspondence $\underline{L}=\left(L_{k(k+1)}\right)_{k=0, \ldots, r}$ satisfying the monotonicity conditions of Theorem 5.2.4. Fix a tuple of widths $\underline{\delta}=$ $\left(\delta_{k}\right)_{k=0, \ldots, r}$. Then Proposition 5.2.1 and Theorem 5.2.4 provide tuples of Hamiltonians $\underline{H}=\left(H_{k}\right)_{k=0 \ldots, r}$ and almost complex structures $\underline{J}=\left(J_{k}\right)_{k=0, \ldots, r}$ such that the Floer homology $H F(\underline{L})$ can be defined by counting quilted Floer trajectories $\underline{u} \in \mathcal{M}\left(\underline{x}^{-}, \underline{x}^{+}\right)$ between generalized intersection points $\underline{x}^{ \pm} \in \mathcal{I}(\underline{L})$.

The proof of independence of Floer cohomology from the choice of perturbations and particularly the widths goes somewhat beyond the proof for standard Floer theory. It is best formulated by using quilted surfaces that are not obtained by "unfolding of strips". With Proposition 5.3.2 below in place we can in particular identify the two definitions of Floer cohomology $H F(\underline{L}) \cong H F\left(L_{(0)}, L_{(1)}\right)$ for a cyclic sequence in Section 4.3 and Section 5.2. For that purpose one chooses special widths in the quilted setup of Section 5.2, namely those that correspond by the discussion in Section 5.1 to the "folded" Floer trajectories of $H F\left(L_{(0)}, L_{(1)}\right)$. The proof of the Proposition however uses the notation and construction of relative quilt invariants in [41]. For readers familiar with this notation, the following Remark describes the quilted Floer trajectories as quilted surfaces. For readers unfamiliar with the quilt notation we will summarize the definition of relative quilt invariants in the special case used in the proof of Proposition 5.3.2 below.

Remark 5.3.1 In the language of quilted surfaces developed in [41] the Floer trajectories correspond to the holomorphic quilted cylinders $\underline{u} \in \mathcal{M}_{\underline{Z}}\left(\underline{x}^{-}, \underline{x}^{+}\right)$with 
$\underline{K}=\left(H_{k} \mathrm{~d} t\right)_{k=0, \ldots, r}$ and $\underline{J}=\left(J_{k}\right)_{k=0, \ldots, r}$. Here the quilted surface is the quilted cylinder $\underline{Z}$ whose patches are strips $\left(S_{k}=\mathbb{R} \times\left[0, \delta_{k}\right]\right)_{k=0, \ldots, r}$ of the given widths with the canonical complex structure and the obvious (up to a shift chosen as \pm 1 ) ends $\epsilon_{k, e_{ \pm}}: \mathbb{R}^{ \pm} \times\left[0, \delta_{k}\right] \rightarrow S_{k},(s, t) \mapsto(s, \pm 1+t)$. The seams are $\sigma_{k}=\left\{\left(k, \mathbb{R} \times\left\{\delta_{k}\right\}\right)\right.$, $(k+1, \mathbb{R} \times\{0\})\}$ for $k=0, \ldots, r \bmod (r+1)$ with seam maps $\phi_{\sigma_{k}}: \partial S_{k} \supset\left(s, \delta_{k}\right) \mapsto$ $(s, 0) \subset \partial S_{k+1}$. This quilted surface is shown on the right in Figure 1. There are no remaining boundary components except for in the special case of a noncyclic sequence with $M_{0}=\{\mathrm{pt}\}$, which is indicated on the left in Figure 1. In that case $\underline{Z}$ has no seam $\sigma_{r}$ between $S_{r}$ and $S_{0}$ but true boundary components $(0, \mathbb{R} \times\{0\})$ and $\left(r, \mathbb{R} \times\left\{\delta_{r}\right\}\right)$. The ends of the quilted surface are the incoming $\underline{e}_{-}=\left(\left(0, e_{-}\right),\left(1, e_{-}\right), \ldots,\left(r, e_{-}\right)\right)$and the outgoing $\underline{e}_{+}=\left(\left(0, e_{+}\right),\left(1, e_{+}\right), \ldots,\left(r, e_{+}\right)\right)$. Note however that the perturbation data $(J, K)$ is $\mathbb{R}$-invariant and the count for the Floer differential is modulo simultaneous $\mathbb{R}$-shift of all maps $u_{k}$. That is, unlike in the definition of relative quilt invariants in [41], where no symmetries are divided out and index 0 solutions are counted, we here count the isolated solutions $\mathcal{M}\left(\underline{x}^{-}, \underline{x}^{+}\right)_{0}=\mathcal{M}_{\underline{Z}}\left(\underline{x}^{-}, \underline{x}^{+}\right)_{1} / \mathbb{R}$, which are pseudoholomorphic quilts of index 1 .

Proposition 5.3.2 $H F(\underline{L})$ is independent, up to isomorphism of $\mathbb{Z}_{N}$-graded groups, of the choice of perturbation data $(\underline{H}, \underline{J})$ and widths $\underline{\delta}$ of the strips.

Proof Suppose that $\left(\underline{H}^{i}, \underline{J}^{i}, \underline{\delta}^{i}\right)$ are two different choices for $i=0,1$. Let $\underline{Z}_{01}$ resp. $\underline{Z}_{10}$ be the quilted cylinder as before, but with complex structures $j_{k}$ on each strip $S_{k} \cong \mathbb{R} \times[0,1]$ that interpolate between the two widths $\delta_{k}^{0}$ and $\delta_{k}^{1}$ at the ends $\left(k, e_{-}\right)$ and $\left(k, e_{+}\right)$, in this order for $\underline{Z}_{01}$ and in reversed order for $\underline{Z}_{10}$. In order for the seams to be real analytic we pick the standard complex structure near the boundary components $\mathbb{R} \times\{0,1\} \subset \partial S_{k}$ and only in the interior of $S_{k}$ scale to the appropriate width and interpolate. Figure 2 shows the example for $r=3$ and $M_{0}=M_{4}=\{\mathrm{pt}\}$. For readers unfamiliar with [41] we specify, as an example, that the quilted surface $\underline{Z}_{01}$ consists of the following data:

(a) A collection $\underline{S}=\left(S_{k}\right)_{k=0, \ldots, r}$ of patches. Here these are the twice punctured disks $S_{k}=\mathbb{R} \times[0,1] \cong D^{2} \backslash\{-1,1\}$ with a complex structure $j_{k}$ and strip-like ends as follows: We fix embeddings of half-strips $\epsilon_{ \pm}: \mathbb{R}^{ \pm} \times\left[0, \delta_{ \pm}\right] \rightarrow S_{k},(s, t) \mapsto\left( \pm 1+\delta_{ \pm}^{-1} s, \delta_{ \pm}^{-1} t\right)$. of width $\delta_{-}=\delta_{k}^{0}$ resp. $\delta_{+}=\delta_{k}^{1}$. These are incoming resp. outgoing strip-like ends with disjoint images. To construct the complex structure we moreover let $\Delta=\frac{1}{3} \min \left(\delta_{+}, \delta_{-}\right)$ and fix disjoint embeddings $\epsilon_{0}: \mathbb{R} \times[0, \Delta] \rightarrow S_{k}$ and $\epsilon_{1}: \mathbb{R} \times[1-\Delta, \Delta] \rightarrow S_{k}$ such that $\epsilon_{0}(s, 0)=(s, 0)$ resp. $\epsilon_{1}(s, 1)=(s, 1)$ and $\epsilon_{0 / 1}^{-1} \circ \epsilon_{ \pm}$are four biholomorphisms with respect to the canonical complex structure on the half-strip and strip. Now we choose a complex structure $j_{k}$ on $S_{k}$ such that it pulls back to the canonical complex structure under each of $\epsilon_{-}, \epsilon_{+}, \epsilon_{0}, \epsilon_{1}$. 
(b) A collection $\mathcal{S}$ of seams. Here these are the set $\mathcal{S}=\left(\sigma_{k}\right)_{k=0, \ldots, r}$ of seam labels $\sigma_{k}=\left\{\left(k, \mathbb{R} \times\{1\} \subset \partial S_{k}\right),\left(k+1, \mathbb{R} \times\{0\} \subset \partial S_{k+1}\right)\right\}$, where we use the label $k$ modulo $(r+1)$, with seam maps $\phi_{\sigma_{k}}: \partial S_{k} \ni(s, 1) \mapsto(s, 0) \in \partial S_{k+1}$ providing diffeomorphisms of boundary components. By our construction these seams are real analytic and compatible with strip-like ends.

(c) Orderings of patches, boundary components, and quilted ends. Here they are given by the enumeration with $k=0, \ldots, r$, and there is only one incoming and one outgoing quilted end consisting of all incoming resp. outgoing strip-like ends on the patches. We

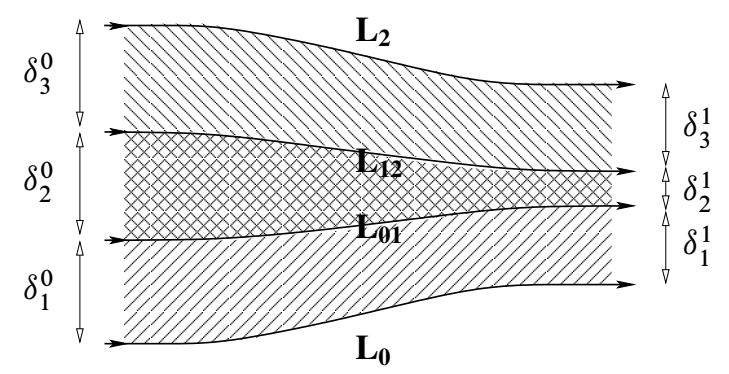

Figure 2: Interpolating between two widths

moreover interpolate the perturbation data on the two ends by some regular $\left(\underline{K}_{01}, \underline{J}_{01}\right)$ on $\underline{Z}_{01}$ and similar for $\underline{Z}_{10}$. Summarizing from [41] this means the following.

(a) We pick function valued one-forms $\underline{K}_{01}=\left(K_{k} \in \Omega^{1}\left(S_{k}, C^{\infty}\left(M_{k}\right)\right)\right)_{k=0, \ldots, r}$ such that $\left.K_{k}\right|_{\partial S_{k}}=0$ and on each end $\epsilon_{-}^{*} K_{k}=H_{k}^{0} \mathrm{~d} t$ resp. $\epsilon_{+}^{*} K_{k}=H_{k}^{1} \mathrm{~d} t$. The corresponding Hamiltonian vector field valued one-forms are denoted by $\underline{Y}_{01}=$ $\left(Y_{k} \in \Omega^{1}\left(S_{k}, \operatorname{Vect}\left(M_{k}\right)\right)\right.$ and satisfy $\epsilon_{ \pm}^{*} Y_{k}=X_{H_{k}^{0 / 1}} \mathrm{~d} t$ on each end.

(b) Let $\mathcal{J}$ denote the set of collections

$$
\underline{J}=\left(J_{k} \in \operatorname{Map}\left(S_{k}, \mathcal{J}\left(M_{k}, \omega_{k}\right)\right)\right)_{k=0, \ldots, r}
$$

of compatible almost complex structures agreeing with the almost complex structures $J_{k}^{0}$ resp. $J_{k}^{1}$ on the incoming ends $\operatorname{im} \epsilon_{-}$resp. the outgoing ends $\operatorname{im} \epsilon_{+}$. We prove in [41] that $\mathcal{J}$ contains an open dense subset of regular $\underline{J}$, of which we pick one $\underline{J}_{01}$.

Given this perturbation data and intersection points $\underline{x}^{-} \in \mathcal{I}(\underline{L})^{0}, \underline{x}^{+} \in \mathcal{I}(\underline{L})^{1}$ we construct in [41] the moduli spaces of pseudoholomorphic quilts $\mathcal{M}_{\underline{Z}_{01}}\left(\underline{x}^{-}, \underline{x}^{+}\right)$and $\mathcal{M}_{\underline{Z}_{10}}\left(\underline{x}^{-}, \underline{x}^{+}\right)$. For example,

$$
\mathcal{M}_{\underline{Z}_{01}}\left(\underline{x}^{-}, \underline{x}^{+}\right):=\left\{\underline{u}=\left(u_{k}: S_{k} \rightarrow M_{k}\right)_{k=0, \ldots, r} \mid(a)-(d)\right\}
$$

is the space of collections of $\left(\underline{J}_{01}, \underline{Y}_{01}\right)$-holomorphic maps with Lagrangian seam conditions, finite energy, and fixed limits, that is 

(a) $J_{k}\left(u_{k}\right) \circ\left(\mathrm{d} u_{k}-Y_{k}\left(u_{k}\right)\right)-\left(\mathrm{d} u_{k}-Y_{k}\left(u_{k}\right)\right) \circ j_{k}=0$ for $k=0, \ldots, r$,
(b) $\left(u_{k}, u_{k+1} \circ \varphi_{\sigma_{k}}\right)(\mathbb{R} \times\{0\}) \subset L_{k(k+1)}$ for all $k=0, \ldots, r$,
(c) $\sum_{k=0}^{r} \int_{S_{k}}\left(u_{k}^{*} \omega_{k}+\mathrm{d}\left(K_{k} \circ u_{k}\right)\right)<\infty$,
(d) $\lim _{s \rightarrow \pm \infty} u_{k}\left(\epsilon_{ \pm}(s, t)\right)=x_{k}^{ \pm}(t)$ for all $k=0, \ldots, r$.

The relative invariants, constructed in [41] from the zero-dimensional moduli spaces of pseudoholomorphic quilts then provide maps between the Floer cohomology groups

$$
\Phi_{\underline{Z}_{01}}: H F(\underline{L})^{0} \rightarrow H F(\underline{L})^{1}, \quad \Phi_{\underline{Z}_{10}}: H F(\underline{L})^{1} \rightarrow H F(\underline{L})^{0} .
$$

We briefly review the construction: On chain level a map $C \Phi_{Z_{01}}: C F(\underline{L})^{0} \rightarrow C F(\underline{L})^{1}$ can be defined by

$$
C \Phi_{\underline{Z}_{01}}\left\langle\underline{x}^{-}\right\rangle:=\sum_{\underline{x}^{+} \in \mathcal{I}(\underline{L})^{1}}\left(\sum_{\underline{u} \in \mathcal{M}_{\underline{z}_{01}}\left(\underline{x}^{-}, \underline{x}^{+}\right)_{0}} \epsilon(u)\right)\left\langle\underline{x}^{+}\right\rangle,
$$

where $\epsilon: \mathcal{M}_{\underline{Z}_{01}}\left(\underline{x}^{-}, \underline{x}^{+}\right)_{0} \rightarrow\{-1,+1\}$ is defined by comparing the orientation constructed in [40] on the zero dimensional component of the moduli space to the canonical orientation of a point. We prove in [41] that the map $C \Phi_{\underline{Z}_{01}}$ is a chain map and so descends to a map of Floer cohomologies. In fact, the map on cohomology level is independent of the choice of perturbation data $\left(\underline{K}_{01}, \underline{J}_{01}\right)$.

Next, the quilted surface $\underline{Z}_{01} \# \underline{Z}_{10}$ that is obtained by gluing the incoming ends of $\underline{Z}_{01}$ to the outgoing ends of $\underline{Z}_{10}$ can be deformed with fixed ends to the infinite strip with translationally invariant complex structures (reflected in the widths $\underline{\delta}^{1}$ ) and perturbation data $\left(\underline{H}^{1}, \underline{J}^{1}\right)$. The relative quilt invariant defined by the latter is the identity on $H F(\underline{L})^{1}$ since only constant strips can contribute (all nonconstant solutions lie in at least 1-dimensional moduli spaces due to the nontriviality of the $\mathbb{R}$-action); see our paper [41]. Since the relative quilt invariants are independent of the above choices, the relative invariant $\Phi_{\underline{Z}_{01} \# \underline{Z}_{10}}$ is the identity on $H F(\underline{L})^{1}$ (and similarly $\Phi_{Z_{10}}^{\# \underline{Z}_{01}}$ is the identity on $\left.H F(\underline{L})^{0}\right)$. Then by the gluing theorem for relative quilt invariants [41] (where the sign is positive) we have

$$
\Phi_{\underline{Z}_{01}} \circ \Phi_{\underline{Z}_{10}}=\Phi_{\underline{Z}_{01} \# \underline{Z}_{10}}=\mathrm{Id}, \quad \Phi_{\underline{Z}_{10}} \circ \Phi_{\underline{Z}_{01}}=\Phi_{\underline{Z}_{10} \# \underline{Z}_{01}}=\text { Id. }
$$

This proves that the Floer cohomology groups $H F(\underline{L})^{0}$ and $H F(\underline{L})^{1}$ arising from the different choices of data are isomorphic. 


\subsection{Geometric composition and quilted Floer cohomology}

In this section we prove and discuss the isomorphism (3), more precisely stated as follows.

Theorem 5.4.1 Let $\underline{L}=\left(L_{01}, \ldots, L_{r(r+1)}\right)$ be a cyclic sequence of Lagrangian correspondences between symplectic manifolds $M_{0}, \ldots, M_{r+1}=M_{0}$ as in Definition 2.1.3. Suppose

(a) the symplectic manifolds all satisfy (M1)-(M2) with the same monotonicity constant $\tau$,

(b) the Lagrangian correspondences all satisfy (L1)-(L2) and at least one of the following:

(i) Each Lagrangian correspondence satisfies (L3).

(ii) The sum $w\left(L_{01}\right)+\cdots+w\left(L_{(j-1) j}\right)+w\left(L_{j(j+1)}\right)+\cdots+w\left(L_{r(r+1)}\right)=0$ of holomorphic disk counts (18) vanishes.

(iii) The sum $w\left(L_{01}\right)+\cdots+w\left(L_{(j-1) j} \circ L_{j(j+1)}\right)+\cdots+w\left(L_{r(r+1)}\right)=0$ of holomorphic disk counts (18) vanishes.

(c) the sequence $\underline{L}$ is monotone, relatively spin and graded in the sense of Section 4.3 ,

(d) the composition $L_{(j-1) j} \circ L_{j(j+1)}$ is embedded in the sense of Definition 2.0.4.

Then with respect to the induced relative spin structure, orientation, and grading ${ }^{9}$ on the modified sequence $\underline{L}^{\prime}=\left(L_{01}, \ldots, L_{(j-1) j} \circ L_{j(j+1)}, \ldots, L_{r(r+1)}\right)$ the following two Floer homologies are well defined and canonically isomorphic as graded groups:

$H F(\underline{L})=H F\left(\ldots L_{(j-1) j}, L_{j(j+1)} \ldots\right) \stackrel{\sim}{\longrightarrow} H F\left(\ldots L_{(j-1) j} \circ L_{j(j+1)} \ldots\right)=H F\left(\underline{L}^{\prime}\right)$.

The isomorphism is canonical in the following sense: The Floer cohomologies defined by any two choices of perturbation data and widths are canonically isomorphic. For sufficiently small width $\delta_{j}>0$ and corresponding perturbation data, the isomorphism $H F(\underline{L}) \cong H F\left(\underline{L}^{\prime}\right)$ is given by the identity map on the generators $\mathcal{I}(\underline{L})=\mathcal{I}\left(\underline{L}^{\prime}\right)$, which are canonically identified by Remark 5.2.2.

Before summarizing the proof let us mention the (im)possibility of various generalizations.

${ }^{9}$ The grading of $L_{(j-1)(j+1)}$ is given by (16), the orientation is given by Remark 2.0.6(b), and for the relative spin structure see our paper [40]. 
Remark 5.4.2 (a) Note the orientation conventions when comparing with the Floer cohomology $H F\left(L_{0}, L_{1}\right)$ for a pair of Lagrangians $L_{0}, L_{1} \subset M$ in [22]. For quilted Floer cohomology, this pair is viewed as cyclic sequence $\{\mathrm{pt}\} \rightarrow M \rightarrow\{\mathrm{pt}\}$, that is the Lagrangian correspondences are $L_{0} \subset\{\mathrm{pt}\}^{-} \times M \cong M$ and $L_{1}^{-} \subset M^{-} \times\{\mathrm{pt}\} \cong M^{-}$, the same submanifold but viewed as Lagrangian with respect to $-\omega$. Thus we obtain $w\left(L_{0}\right)+w\left(L_{1}^{-}\right)=w\left(L_{0}\right)-w\left(L_{1}\right)$, since the $-J_{1}$-holomorphic discs with boundary on $L_{1}^{-} \subset M^{-}$are identified with $J_{1}$-holomorphic discs with boundary on $L_{1} \subset M$ via a reflection of the domain, which is orientation reversing for the moduli spaces.

(b) If in Theorem 5.4.1 (b) we only assume (L1)-(L2) then Floer cohomology may not be well-defined due to $\partial^{2}=w \mathrm{Id}$, where $w=w\left(L_{01}\right)+\cdots+w\left(L_{r(r+1)}\right)[22 ; 43]$. In case $w \neq 0$ the chain homotopy equivalence $(C F(\underline{L}), \partial) \cong\left(C F\left(\underline{L}^{\prime}\right), \partial^{\prime}\right)$ continues to hold in the derived category of matrix factorizations ${ }^{10}$ [43].

We will show that either of the extra assumptions (i), (ii) or (iii) implies that $\partial^{2}=0$ on both Floer complexes, and hence implies both (ii) and (iii) (but not (i)). In fact, (i) directly implies (ii) (but not (iii)) since all minimal Maslov numbers being at least 3 implies $w\left(L_{(i-1) i}\right)=0$ for each $i$. The other implications (i) $\Rightarrow$ (iii), (ii) $\Rightarrow$ (iii), (iii) $\Rightarrow$ (ii) also require the monotonicity and embeddedness assumptions since they follow indirectly from the above isomorphism in the derived category.

(c) The relative spin structures are only needed to define the Floer cohomology groups with $\mathbb{Z}$ coefficients. Here we only prove the isomorphism with $\mathbb{Z}_{2}$ coefficients. The full result then follows from a comparison of signs in [40].

(d) There should also be versions of this result for Floer cohomology with coefficients in flat vector bundles, and Novikov rings, using an understanding of their behaviour under geometric composition, similar to the theory presented here for gradings. The gradings on the Lagrangians can be dropped if one wants only an isomorphism of ungraded groups.

(e) Note that the geometric composition $L_{(j-1) j} \circ L_{j(j+1)}$ could be a smooth Lagrangian despite the composition not being embedded. If this failure is in the transversality, then our approach does not apply (as eg for a $G$-invariant Lagrangian $L \subset \mu^{-1}(0)$ in the zero set of the moment map, whose composition with $\Sigma_{\mu}$ is the smooth projection $L \circ \Sigma_{\mu}=\pi(L)$ despite $L$ not being transverse to $\mu^{-1}(0)$ ). (For such Lagrangians one would expect a correspondence between holomorphic curves in $M / / G$ and symplectic vortices in $M$, in the spirit of [11] and the Lagrangian version of the Atiyah-Floer conjecture [37].) However, when $L_{(j-1) j} \times_{M_{j}} L_{j(j+1)}$

${ }^{10}$ Explicitly, there exist chain maps $f: C F(\underline{L}) \rightarrow C F\left(\underline{L}^{\prime}\right)$ and $g: C F\left(\underline{L}^{\prime}\right) \rightarrow C F(\underline{L})$ such that both $f \circ g$ and $g \circ f$ are homotopy equivalent to the identity in the sense that eg $\operatorname{Id}-g \circ f=k \partial-\partial k$ for some $k: C F(\underline{L}) \rightarrow C F(\underline{L})$. 
is transverse but a $k$-fold cover of $L_{(j-1) j} \circ L_{j(j+1)}$, then the map of intersection points $\mathcal{I}(\underline{L}) \rightarrow \mathcal{I}\left(\underline{L}^{\prime}\right)$ is a $k$-to-1 map as well. In this case our analysis still applies and gives a $k$-to-1 map of moduli spaces as long as bubbling is excluded. This leads to further calculation tools for Floer cohomology but needs to be investigated on a case-by-case basis.

(f) The monotonicity assumptions (M1) and (L1) cannot simply be replaced by other tools which allow the definition of Floer cohomology (such as Novikov rings, twisted coefficients, obstructions, or deformations). This is since a new type of bubbling can occur in the strip shrinking that we use to prove the isomorphism. We have called it the "figure eight bubble" and describe it in [38]. However, we are lacking the construction of a moduli space of figure eight bubbles. Our present method for excluding these bubbles hinges on strict monotonicity with nonnegative constant $\tau \geq 0$ as well as the 2-grading assumption implied by orientations. In general, we expect figure eight bubbles to be a codimension 1 phenomenon in a 1-parameter family of strip widths approaching zero. We hence expect the isomorphism to fail in more general settings, except for special topological assumptions restricting the expected dimension of the moduli space of figure eight bubbles. Eventually, we expect to construct obstruction classes and an $A_{\infty}$-type structure from moduli spaces of figure eight bubbles, and to replace the isomorphism by a morphism of $A_{\infty}$-modules. However, all of this depends on a basic removable singularity result for figure eight bubbles, which has not yet been accomplished.

Theorem 5.4.1 is fairly obvious if one of the composed Lagrangians correspondences is the graph of a symplectomorphism. It suffices to observe that symplectomorphisms map pseudoholomorphic curves to pseudoholomorphic curves. However, there is no corresponding effect for more general Lagrangian correspondences. Here the natural approach to a proof is to degenerate the holomorphic curve equation in $M_{j}$ until solutions become constant across the strip (or, equivalently, shrink the width of that strip to zero). This limit corresponds to geometric composition of the two Lagrangian correspondences attached to the strip. Clearly, most difficulties in this proof are localized near the degenerating strip. We thus banished the analysis to [38], where we prove the special case $H F\left(L_{0}, L_{01}, L_{12}, L_{2}\right) \stackrel{\sim}{\rightarrow} H F\left(L_{0}, L_{01} \circ L_{12}, L_{2}\right)$ of Theorem 5.4.1 by establishing a bijection between the Floer trajectories for $\left(L_{0}, L_{02}, L_{2}\right)$ on strips of width $(1,1)$ and those for $\left(L_{0}, L_{01}, L_{12}, L_{2}\right)$ on strips of width $(1, \delta, 1)$ for sufficiently small width $\delta$ of the middle strip. These quilted Floer trajectories are shown in Figure 3. The missing piece of proof in [38] is the independence of the Floer cohomology from the choice of $\delta>0$, which we here established in Proposition 5.3.2. 

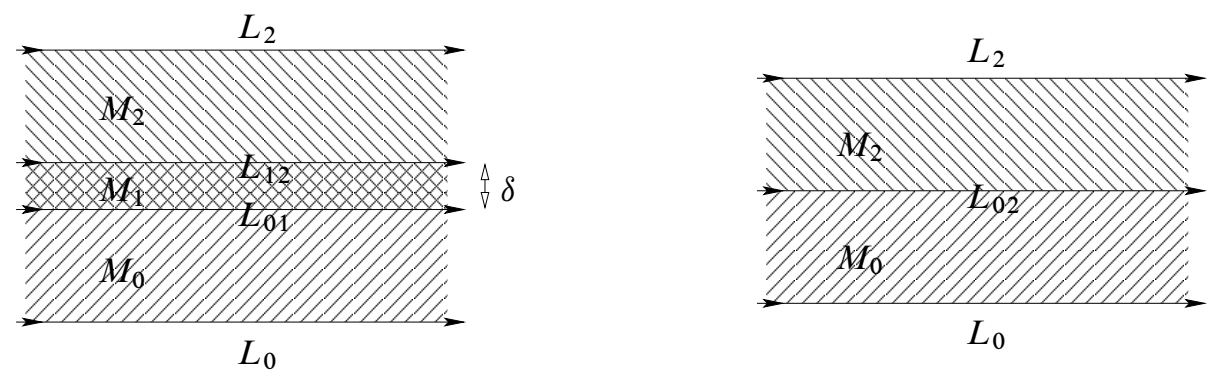

Figure 3: Shrinking the middle strip

Summary of proof of Theorem 5.4.1 We first consider the case of assumption (i) or (ii) holding in (b). Then the assumptions of the Theorem guarantee that $H F(\underline{L})$ is well-defined. By Remark 5.2.3 the monotonicity of $\underline{L}$ also implies monotonicity of $\underline{L}^{\prime}$ and hence monotonicity in the sense of (L1) for $L_{(j-1) j} \circ L_{j(j+1)}$. (Assuming the symplectic manifolds to be connected, any disk can be extended to a quilted cylinder.) Compactness and orientation (L2) also holds for the composed correspondence, but the minimal Maslov index condition (L3) may not transfer. However, this only affects the question whether $\partial^{2}=w \mathrm{Id}=0$ on the Floer chain group for $\underline{L}^{\prime}$. In fact, we just assumed that $w=w\left(L_{01}\right)+\cdots+w\left(L_{r(r+1)}\right)=0$ on $C F(\underline{L})$, and a priori it is not clear that one should have $w\left(L_{(j-1) j} \circ L_{j(j+1)}\right)=w\left(L_{(j-1) j}\right)+w\left(L_{j(j+1)}\right)$. If, on the other hand, assumption (iii) holds in (b), then we are guaranteed that $H F\left(\underline{L}^{\prime}\right)$ is well-defined, but it is not a priori clear that $\partial^{2}=0$ on $C F(\underline{L})$.

In either case, to define the differential on $C F(\underline{L})$ we choose some widths $\underline{\delta}^{\prime}$, Hamiltonian perturbations $\underline{H}^{\prime}$ to make the intersection $\mathcal{I}\left(\underline{L}^{\prime}\right)$ transverse, and almost complex structures $\underline{J}^{\prime}$ to make the moduli spaces of Floer trajectories for $\underline{L}^{\prime}$ regular. Thanks to Proposition 5.3.2 we may then choose the same widths $\underline{\delta}$ except for some small $\delta_{j}>0$, the same Hamiltonian perturbations $\underline{H}$ except for the additional $H_{j} \equiv 0$, and the same almost complex structures $\underline{J}$ except for some additional time-independent $J_{j} \in \mathcal{J}\left(M_{j}, \omega_{j}\right)$, to define $H F(\underline{L})$. We only need to make sure that this choice makes the intersection points $\mathcal{I}(\underline{L})$ and the moduli spaces of Floer trajectories for $\underline{L}$ regular. The first is automatically the case by the transversality assumption for $L_{(j-1) j} \times_{M_{j}} L_{j(j+1)}$, the latter is true for $\delta_{j}>0$ sufficiently small and is proven as part of the adiabatic limit analysis [38]. (Actually, precisely following the constructions of [38], we can achieve transversality for $\underline{L}^{\prime}$ with $J_{j-1}^{\prime}$ and $J_{j+1}^{\prime}$ being time-independent near the seam; then $J_{j-1}$ and $J_{j+1}$ are obtained by a slight linear dilation and constant extension near the new seams.) With these choices, our assumption (b) on holomorphic disk counts (or (L3) on the minimal Maslov index) implies $\partial^{2}=0$ on $C F(\underline{L})$. 
Next, the injectivity assumption for the composition $L_{(j-1) j} \times_{M_{j}} L_{j(j+1)}$ provides a canonical bijection of generalized intersection points $\mathcal{I}\left(\underline{L}^{\prime}\right) \cong \mathcal{I}(\underline{L})$ as in Remark 3.1.3. In [38] we establish bijections between the corresponding moduli spaces of Floer trajectories for $\delta_{j}>0$ sufficiently small. This means that the Floer differentials on $C F\left(\underline{L}^{\prime}\right)$ and $C F(\underline{L})$ agree under the canonical identification of generators. In particular that implies $\partial^{2}=0$ on both complexes as soon as it is true on one (which is ensured by each version of assumption (b)). Hence both Floer cohomologies are well-defined and isomorphic as claimed. (In fact, we deduce a posteriori that $w\left(L_{(j-1) j} \circ L_{j(j+1)}\right)=$ $\left.w\left(L_{(j-1) j}\right)+w\left(L_{j(j+1)}\right).\right)$

Remark 5.4.3 To see that the assumption that the composition $L_{(j-1) j} \circ L_{j(j+1)}$ is embedded is necessary, consider the case that $r=2$ and $M_{0}, M_{2}$ are points. In this case, if $v: \mathbb{R} \times[0,1] \rightarrow M_{1}$ is a Floer trajectory of index one with limits $x^{+} \neq x^{-}$, we can consider the rescaled maps $v_{\delta}: \mathbb{R} \times[0, \delta] \rightarrow M_{1}$. In this case a figure eight bubble always develops in the limit $\delta \rightarrow 0$. This shows that the bijection between trajectories fails in this case.

\section{Applications}

Quilted Floer homology was originally designed to construct symplectic versions of gauge theoretic invariants, in particular symplectic versions of Donaldson invariants, which we develop in later papers [42; 43], Seiberg-Witten invariants as in Perutz [25] and Lekili [16], and Khovanov invariants as in Rezazadegan [26]. Applications to symplectic topology are given for moduli spaces of flat bundles in [43], and to classification of Lagrangians in tori in Abouzaid-Smith [1]. In this section, we give a few brief applications of the main result to symplectic topology, which show how the results work in practice. Most of the concrete examples can be achieved with other, less sophisticated methods. Our point in giving them is to show how many Floer homology calculations can be obtained from a single principle: Floer homology is well defined under embedded geometric composition. The reader looking for more sophisticated applications is encouraged to look at the sequel papers and the references above.

\subsection{Direct computation of Floer cohomology}

Theorem 6.1.1 Let $L_{01} \subset M_{0}^{-} \times M_{1}$ be a Lagrangian correspondence and suppose that the Lagrangian submanifolds $L_{0} \subset M_{0}$ and $L_{1} \subset M_{1}$ are such that both $L_{0} \circ L_{01}$ and $L_{01} \circ L_{1}$ are embedded compositions. Assume that $M_{0}, M_{1}$ satisfy (M1)-(M2), $L_{0}, L_{1}, L_{01}$ satisfy (L1)-(L2), and $\left(L_{0} \times L_{1}, L_{01}\right)$ is a monotone pair in the sense of 
Definition 4.1.2 (b). Then there exists a canonical isomorphism

$$
C F\left(L_{0} \circ L_{01}, L_{1}\right) \stackrel{\sim}{\longrightarrow} C F\left(L_{0}, L_{01} \circ L_{1}\right)
$$

in the category of derived matrix factorizations. It is an isomorphism of Floer cohomologies if one of the following holds: All Lagrangians satisfy (L3), or $w\left(L_{0}\right)+$ $w\left(L_{01}\right)+w\left(L_{1}\right)=0$, or $w\left(L_{0} \circ L_{01}\right)+w\left(L_{1}\right)=0$, or $w\left(L_{0}\right)+w\left(L_{01} \circ L_{1}\right)=0$.

Proof By Theorem 5.4.1 both Floer cohomologies are isomorphic to the quilted Floer cohomology $H F\left(L_{0}, L_{01}, L_{1}\right)=H F\left(L_{0} \times L_{1}, L_{01}\right)$. In case $\partial^{2} \neq 0$, the isomorphism of homologies is replaced by a chain homotopy equivalence in the derived category; see Remark 5.4.2.

Example 6.1.2 We begin with a "warm-up" example. Let $N$ be a compact, simply connected, monotone symplectic manifold. The submanifold

$$
\Delta_{i j}:=\left\{\left(x_{1}, x_{2}, x_{3}, x_{4}\right) \mid x_{i}=x_{j}\right\} \subset N^{-} \times N \times N^{-} \times N
$$

is coisotropic for appropriate choices of $1 \leq i<j \leq 4$. Then we can identify

$$
H F\left(\Delta_{14} \cap \Delta_{23}, \Delta_{12} \cap \Delta_{34}\right) \cong H F\left(\Delta_{N}, \Delta_{N}\right) \cong H(N)
$$

with the homology of $N$. This follows from Theorem 6.1.1 applied to $L_{0}=\Delta_{N} \subset$ $N^{-} \times N=M_{0}, L_{1}=\Delta_{12} \cap \Delta_{34} \subset N^{-} \times N \times N^{-} \times N=M_{1}$, and $L_{01}=$ $\{(w, z ; w, x, x, z) \mid w, x, z \in N\} \subset M_{0}^{-} \times M_{1}$. Then the compositions $L_{0} \circ L_{01}=$ $\Delta_{14} \cap \Delta_{23}$ and $L_{01} \circ L_{1}=\Delta_{N}$ are clearly embedded. Monotonicity together with simply connectedness ensures the monotonicity of all the Lagrangians and pairs of Lagrangians. Since $N$ is orientable, all minimal Maslov indices are at least 2. The reader can easily verify the identification (31) using the fact that the components of a holomorphic trajectory for $\left(\Delta_{14} \cap \Delta_{23}, \Delta_{12} \cap \Delta_{34}\right)$ fit together to a holomorphic cylinder $v: S^{1} \times[0,1] \rightarrow N$.

The following is a more nontrivial example of Theorem 6.1.1.

Example 6.1.3 Let $M_{n}$ be the moduli space of Euclidean $n$-gons of edge length 1, as in for example Kirwan [13]:

$$
M_{n}=\left(S^{2}\right)^{n} / / \mathrm{SO}(3)=\left\{\left(v_{1}, \ldots, v_{n}\right) \in\left(S^{2}\right)^{n} \mid v_{1}+\cdots+v_{n}=0\right\} / \mathrm{SO}(3) .
$$

We take on $S^{2}$ the standard symplectic form $\omega$ with volume $4 \pi$ so that $c_{1}\left(S^{2}\right)=$ [ $\omega$ ]. For $n \geq 5$ odd $M_{n}$ is a monotone symplectic manifold with minimal Chern number 1 and monotonicity constant 1 . For example, $M_{3}$ is a point and $M_{5}$ is diffeomorphic to the fourth del Pezzo surface, given by blowing-up of $\mathbb{P}^{2}$ at four points 
[34, Example 1.10]. For $i \neq j$ the submanifold $\Delta_{i j}=\left\{\left[v_{1}, \ldots, v_{n}\right] \in M_{n} \mid v_{i}=-v_{j}\right\}$ is a coisotropic, spherically fibered over $M_{n-2}$ by the map that forgets $v_{i}, v_{j}$. The image of $\Delta_{i j}$ in $M_{n-2}^{-} \times M_{n}$ is a Lagrangian correspondence, also denoted $\Delta_{i j}$. For $i, j, k$ distinct the composition $\Delta_{i j} \circ \Delta_{j k}^{t}$ is embedded and yields the graph of a permutation on $M_{n-2}$. For $k=i \pm 1$ or for $i, k=j \pm 1$ this permutation is trivial, so we have

$$
\Delta_{i j} \circ \Delta_{j(i \pm 1)}^{t}=\Delta_{M_{n-2}}, \quad \Delta_{(j \pm 1) j} \circ \Delta_{j(j \mp 1)}^{t}=\Delta_{M_{n-2}} .
$$

Now let $L \subset M_{n-2}$ be a compact, oriented, monotone Lagrangian, and $L_{i j}=L \circ \Delta_{i j}$ be its inverse image in $M_{n}$. This composition is embedded and we can also identify it with $L_{i j}=\Delta_{i j}^{t} \circ L$. The latter allows to calculate

$$
\Delta_{i j} \circ L_{j k}=\Delta_{i j} \circ \Delta_{j k}^{t} \circ L \text {. }
$$

For $i, j, k$ distinct it is an embedded composition, which yields the image of $L$ under permutation. Suppose that the pair $(L, L)$ is monotone, so that $\operatorname{HF}(L, L)$ is well-defined. Using Theorem 6.1.1 we can also calculate

$$
H F\left(L_{i j}, L_{j(i \pm 1)}\right)=H F\left(L \circ \Delta_{i j}, L_{j(i \pm 1)}\right)=H F\left(L, \Delta_{i j} \circ L_{j(i-1)}\right)=H F(L, L) .
$$

Here all Floer homologies are defined since $\partial^{2}=0$ on $C F(L, L)$. Similarly, we obtain $H F\left(L_{(j \pm 1) j}, L_{j(j \mp 1)}\right)=H F(L, L)$.

The symplectic manifolds in the above example are certain moduli spaces of parabolic bundles on punctured spheres, which can also be seen as SU(2)-representation spaces of punctured spheres with fixed conjugacy class for each puncture [19]. The Lagrangian submanifolds $\Delta_{i j}$ arise from elementary tangles connecting two punctures, by taking parabolic bundles on the corresponding cobordism of punctured spheres, and restricting them to the boundary. The fact that their composition is the graph of a permutation reflects the fact that all braid moves on the punctures can be decomposed into elementary tangles. Thus one may hope to define knot or tangle invariants by decomposition into elementary tangles and representation in the symplectic category as above. The invariant should then be the quilted Floer cohomology of the sequence of Lagrangian correspondences arising from the sequence of elementary tangles. In order to prove invariance, one needs to check that moves between different decompositions are reflected by isomorphisms of Floer cohomology. The above example shows how this follows from our main Theorem for the cancellation of two elementary tangles.

This general approach to defining topological invariants is also described in the introduction. The moduli spaces in Example 6.1.3 actually yield the trivial invariant since they arise from conjugacy classes close to the center of SU(2). In [42; 43] we 
employ similar moduli spaces with different conjugacy classes, giving rise to nontrivial invariants of tangles and 3-manifolds.

\subsection{Computations in $\mathbb{C P}^{n}$}

In this section we demonstrate, at the example of $\mathbb{C} \mathbb{P}^{n}$, how some Floer cohomologies in toric symplectic varieties can be calculated by reduction. We equip $\mathbb{C P} \mathbb{P}^{n}=\{[z]=$ $\left.\left[z_{0}: z_{1}: \cdots: z_{n}\right]\right\}$ with Fubini-Study symplectic form and moment maps $\mu_{j}([\underline{z}])=$ $\pi\left|z_{j}\right|^{2} /|\underline{z}|^{2}$ for $j=1, \ldots, n$. We denote by

$$
\begin{aligned}
\Sigma_{j} & :=\left\{\left(\left[z_{0}: \ldots z_{j-1}: z_{j+1}: \cdots: z_{n}\right],\left[z_{0}: \cdots: z_{n}\right]\right) \mid \mu_{j}\left(\left[z_{0}: \cdots: z_{n}\right]\right)=\frac{\pi}{n+1}\right\} \\
& \subset\left(\mathbb{C} \mathbb{P}^{n-1}\right)^{-} \times \mathbb{C P}^{n}
\end{aligned}
$$

the Lagrangian sphere arising from reduction at the level set

$$
\mu_{j}^{-1}\left(\frac{\pi}{n+1}\right)=\left\{\left.\left[z_{0}: \cdots: z_{n}\right]\left|z_{j}=\frac{1}{\sqrt{n+1}}, \sum_{i \neq j}\right| z_{i}\right|^{2}=\frac{n}{n+1}\right\} .
$$

Note that the reduced space, eg

$$
\mu_{n}^{-1}\left(\frac{\pi}{n+1}\right) / S^{1}=\left\{\left.\left[z_{0}: \ldots z_{n-1}\right]\left|\sum_{i}\right| z_{i}\right|^{2}=\frac{n}{n+1}\right\} / S^{1}
$$

for $j=n$, is $\mathbb{C} \mathbb{P}^{n-1}$ with Fubini-Study form scaled by $\frac{n}{n+1}$, hence has the same monotonicity constant $\tau=n^{-1} \frac{n \pi}{n+1}=(n+1)^{-1} \pi$ as $\mathbb{C} \mathbb{P}^{n}$. (Recall that the generator $\mathbb{C} \mathbb{P}^{1} \subset \mathbb{C P}^{n}$ of $\pi_{2}\left(\mathbb{C} \mathbb{P}^{n}\right)$ has Fubini-Study symplectic area $\pi$ and Chern number $n+1$.

More generally, for each $1<k \leq n$ a Lagrangian correspondence

$\Sigma_{(k, \ldots, n)}:=\left\{\left(\left[z_{0} \ldots z_{k-1}\right],\left[z_{0} \ldots z_{n}\right]\right) \mid \mu_{j}([\underline{]}])=\frac{\pi}{n+1} \forall j \geq k\right\} \subset\left(\mathbb{C} \mathbb{P}^{k-1}\right)^{-} \times \mathbb{C} \mathbb{P}^{n}$ arises from reduction at the level set

$$
\begin{aligned}
\left(\mu_{k} \times \cdots \mu_{n}\right)^{-1}\left(\frac{\pi}{n+1}, \ldots\right. & \left.\frac{\pi}{n+1}\right) \\
& =\left\{\left.[z]\left|z_{k}=\right| z_{k+1}|=\ldots| z_{n}\left|=\frac{1}{\sqrt{n+1}}, \sum_{i=0}^{k-1}\right| z_{i}\right|^{2}=\frac{k}{n+1}\right\} .
\end{aligned}
$$

Here again the reduced spaces $\mathbb{C P}^{k-1}=\left\{\left.\left[z_{0}: \cdots: z_{k-1}\right]\left|\sum_{i}\right| z_{i}\right|^{2}=\frac{k}{n+1}\right\} / S^{1}$ carry scaled Fubini-Study forms with monotonicity constant $\tau=\frac{\pi}{n+1}$. Moreover, note that $\Sigma_{(k, \ldots, n)}$, diffeomorphic to the product of an $(n-k)$-torus with a $(2 k-1)$-sphere, can be viewed as Lagrangian embedded in $\left(\mathbb{C P}^{k-1}\right)^{-} \times \mathbb{C P}^{n}$ and also as coisotropic submanifold of $\mathbb{C} \mathbb{P}^{n}$. One can check explicitly that the Lagrangians $\Sigma_{(k, \ldots, n)}$ are monotone, and we will see in Corollary 6.3 .3 below that they are nondisplaceable 
by Hamiltonian diffeomorphisms. The reason is that as coisotropic they contain the nondisplaceable Clifford torus

$T_{\mathrm{Cl}}^{n}=\left(\mu_{1} \times \cdots \mu_{n}\right)^{-1}\left(\frac{\pi}{n+1}, \ldots, \frac{\pi}{n+1}\right)=\left\{[\underline{z}]\left|z_{0}=\right| z_{1}|=\ldots=| z_{n} \mid=\frac{1}{\sqrt{n+1}}\right\} \subset \mathbb{C P}^{n}$.

That $T_{\mathrm{Cl}}^{n}$ is the only nondisplaceable fibre of the torus fibration is known by eg [3]. Its Floer cohomology was calculated by Cho [4] with all possible spin structures. Here we reproduce this calculation for the standard spin structure, employing the above Lagrangian correspondences and the isomorphism of Floer cohomology under embedded geometric composition (Theorem 5.4.1). This approach also allows for a direct computation of Floer cohomology for any pair of nonstandard spin structures on $T_{\mathrm{Cl}}^{n}$, which we will discuss in [40].

Theorem 6.2.1 [4] For any $n \in \mathbb{N}$ with the standard spin structure (given by [4, Proposition 8.1])

$$
H F\left(T_{\mathrm{Cl}}^{n}, T_{\mathrm{Cl}}^{n}\right) \cong H_{*}\left(T^{n}\right) \cong \mathbb{Z}^{2^{n}}
$$

Proof The isomorphism between the Floer cohomology and the homology of the Clifford $n$-torus follows inductively from the following chain of isomorphisms:

$$
\begin{aligned}
H F\left(T_{\mathrm{Cl}}^{n}, T_{\mathrm{Cl}}^{n}\right) & =H F\left(T_{\mathrm{Cl}}^{1} \circ \Sigma_{(2, \ldots, n)}, \Sigma_{1}^{t} \circ T_{\mathrm{Cl}}^{n-1}\right) \\
& \cong H F\left(T_{\mathrm{Cl}}^{1}, \Sigma_{(2, \ldots, n)}, \Sigma_{1}^{t}, T_{\mathrm{Cl}}^{n-1}\right) \\
& \cong H F\left(T_{\mathrm{Cl}}^{1}, \Sigma_{(2, \ldots, n)} \circ \Sigma_{1}^{t}, T_{\mathrm{Cl}}^{n-1}\right) \\
& \cong H F\left(T_{\mathrm{Cl}}^{1}, T_{\mathrm{Cl}}^{1} \times T_{\mathrm{Cl}}^{n-1}, T_{\mathrm{Cl}}^{n-1}\right) \\
& \cong H F\left(T_{\mathrm{Cl}}^{1}, T_{\mathrm{Cl}}^{1}\right) \otimes H F\left(T_{\mathrm{Cl}}^{n-1}, T_{\mathrm{Cl}}^{n-1}\right)
\end{aligned}
$$

Let us go through this step by step: The geometric composition $T_{\mathrm{Cl}}^{1} \circ \Sigma_{(2, \ldots, n)}=T_{\mathrm{Cl}}^{n}$ is the preimage of $T_{\mathrm{Cl}}^{1}$ under the projection $\left(\mu_{2} \times \cdots \times \mu_{n}\right)^{-1}\left(\frac{\pi}{n+1}, \ldots, \frac{\pi}{n+1}\right) \rightarrow \mathbb{C P}^{1}$, hence automatically embedded in the sense of Definition 2.0.4. Similarly, $T_{\mathrm{Cl}}^{n-1} \circ \Sigma_{1}=$ $T_{\mathrm{Cl}}^{n}$ is the preimage of $T_{\mathrm{Cl}}^{n-1}$ under the projection $\mu_{1}^{-1}\left(\frac{\pi}{n+1}\right) \rightarrow \mathbb{C} \mathbb{P}^{n-1}$, and by transposition we obtain the embedded composition $\Sigma_{1}^{t} \circ T_{\mathrm{Cl}}^{n-1}=T_{\mathrm{Cl}}^{n}$. Next, the intersection

$\Sigma_{(2, \ldots, n)} \times \mathbb{C P}^{n} \Sigma_{1}^{t} \cong\left(\mu_{2} \times \cdots \times \mu_{n}\right)^{-1}\left(\frac{\pi}{n+1}, \ldots, \frac{\pi}{n+1}\right) \cap \mu_{1}^{-1}\left(\frac{\pi}{n+1}\right)=T_{\mathrm{Cl}}^{n} \subset \mathbb{C P}^{n}$ is transverse and embeds to

$$
\Sigma_{(2, \ldots, n)} \circ \Sigma_{1}^{t}=\left\{\left(\left[z_{0}: z_{1}\right],\left[z_{0}: z_{2}: \cdots: z_{n}\right]\right) \mid[z] \in T_{\mathrm{Cl}}^{n}\right\}=T_{\mathrm{Cl}}^{1} \times T_{\mathrm{Cl}}^{n-1} \subset \mathbb{C P}^{1} \times \mathbb{C P}^{n-1}
$$

To make sure that Theorem 5.4.1 indeed implies all the above isomorphisms of Floer cohomology, it remains to ensure that the tuple $\left(T_{\mathrm{Cl}}^{1}, \Sigma_{(2, \ldots, n)}, \Sigma_{j}^{t}, T_{\mathrm{Cl}}^{n-1}\right)$ is monotone. 
That follows from the monotonicity of all factors together with the torsion fundamental groups of the symplectic manifolds involved. Moreover, it turns out that we need not worry about the minimal Maslov indices 2 . This is since we have $\partial^{2}=0$ on the first chain group $C F\left(T_{\mathrm{Cl}}, T_{\mathrm{Cl}}\right)$, ie assumption (b)(iii) is satisfied (see Oh [22] and note Remark 5.4.2 (a)). Now Theorem 5.4.1 implies $\partial^{2}=0$ (ie (b)(ii) resp. (iii)) for each of the other chain groups in (32).

Moreover, we need to fix spin structures on $T_{\mathrm{Cl}}^{n-1}$ and $\Sigma_{1}$ as well as on $T_{\mathrm{Cl}}^{1}$ and $\Sigma_{(2, \ldots, n)}$ such that the induced spin structure on the composition, $T_{\mathrm{Cl}}^{n}$ is the standard one. For the Clifford tori we pick the standard spin structure given by the trivialization of $T T_{\mathrm{Cl}}^{k} \subset \mathbb{C}^{k}$ in the coordinate chart $\mathbb{C}^{k} \cong\left\{z_{0}=1 / \sqrt{n+1}\right\} \subset \mathbb{C P}^{k}$. On the sphere $\Sigma_{1} \subset \mathbb{C} \mathbb{P}^{n}$ we fix the spin structure given by the standard orientation in the chart $\left\{z_{1}=1 / \sqrt{n+1}\right\}$. (The orientation provides a trivialization over the 0 -skeleton, which coincides with the $1-$ and 2 -skeleton of this sphere of dimension $\geq 3$; see Cho [4] or our paper [40] for more details on spin structures.) We can read off the standard spin structure induced on $T_{\mathrm{Cl}}^{n}$ from the identification

$$
\mathrm{T} T_{\mathrm{Cl}}^{n} \cong \mathrm{pr}^{*} \mathrm{~T}_{\mathrm{Cl}}^{n-1} \oplus E, \quad E=\left.\left(\mathrm{pr}^{*} \mathrm{~T} \mathbb{C} \mathbb{P}^{n-1}\right)^{\perp} \subset \mathrm{T} \Sigma_{1}\right|_{\mathrm{T}_{\mathrm{Cl}}^{\mathrm{n}}} .
$$

Here $\left.\mathrm{T} C \mathbb{P}^{n-1}\right|_{T_{\mathrm{Cl}}^{n-1}}=\mathrm{T} T_{\mathrm{Cl}}^{n-1} \oplus i \mathrm{~T} T_{\mathrm{Cl}}^{n-1}$ inherits a trivialization from $T_{\mathrm{Cl}}^{n-1}$, so the orientation of $\Sigma_{1}$ induces a trivialization of the line bundle $E$ (given by the linearized action of $\left.\mu_{1}\right)$.

For the spin structure on $\Sigma_{(2, \ldots, n)} \subset \mathbb{C P}^{n}$ we identify $\Sigma_{(2, \ldots, n)} \cong T^{n-2} \cdot S^{3}$ with the orbit of the sphere

$$
S^{3}=\left\{\left.\left[z_{0}: z_{1}: \frac{1}{\sqrt{n+1}}: \cdots: \frac{1}{\sqrt{n+1}}\right]|| z_{0}\right|^{2}+\left|z_{1}\right|^{2}=\frac{2}{n+1}\right\} \subset \mathbb{C P}^{n}
$$

under the action of the torus $T^{n-2} \subset \mathbb{C}^{n-2}$ in the $z_{3}, \ldots, z_{n}$-coordinates. If we pick the standard trivialization of $T^{n-2}$ and the standard orientation of $S^{3} \subset \mathbb{C}^{2}$ in the above chart, then again the standard spin structure is induced on $T_{\mathrm{Cl}}^{n}$ by the identification

$$
\mathrm{T}_{\underline{z}} T_{\mathrm{Cl}}^{n} \cong \mathrm{T}_{\mathrm{pr}(\underline{z})} T_{\mathrm{Cl}}^{1} \oplus \mathrm{T}_{\underline{z}}\left(T^{n-2} \underline{z}\right) \oplus F_{\left(z_{0}, z_{1}\right)}, \quad F=\left.\left(\operatorname{pr}^{*} \mathrm{~T} \mathbb{C} \mathbb{P}^{1}\right)^{\perp} \subset \mathrm{T} S^{3}\right|_{T_{\mathrm{Cl}}^{1}}
$$

Here $\left.\mathrm{T} C \mathbb{P}^{1}\right|_{T_{\mathrm{Cl}}^{1}}=\mathrm{T} T_{\mathrm{Cl}}^{1} \oplus i \mathrm{~T} T_{\mathrm{Cl}}^{1}$ inherits a trivialization from $T_{\mathrm{Cl}}^{1}$, so the orientation of $S^{3}$ induces a trivialization of the line bundle $F$.

The last isomorphism $H F\left(T_{\mathrm{Cl}}^{1}, T_{\mathrm{Cl}}^{1} \times T_{\mathrm{Cl}}^{n-1}, T_{\mathrm{Cl}}^{n-1}\right) \cong H F\left(T_{\mathrm{Cl}}^{1}, T_{\mathrm{Cl}}^{1}\right) \otimes H F\left(T_{\mathrm{Cl}}^{n-1}, T_{\mathrm{Cl}}^{n-1}\right)$ in (32) follows from the Künneth Theorem 5.2.6. Here the right hand side is the tensor product of homologies since the first factor is torsion-free. We know from elementary curve counts (see eg Cho [4]) that, with the standard spin structure on both factors, $H F\left(T_{\mathrm{Cl}}^{1}, T_{\mathrm{Cl}}^{1}\right) \cong \mathbb{Z} \oplus \mathbb{Z} \cong H_{*}\left(S^{1}=T^{1}\right)$. Finally, the homology $H_{*}\left(T^{n}\right)$ satisfies the same inductive relation (32) as the Floer cohomology. This proves the theorem. 
This Floer cohomology calculation directly generalizes when replacing $T_{\mathrm{Cl}}^{1} \subset \mathbb{C} \mathbb{P}^{1}$ with another Lagrangian submanifold in a possibly higher dimensional complex projective space.

Theorem 6.2.2 Let $1 \leq k<n$ and let $L \subset \mathbb{C P}^{k}$ and $L^{\prime} \subset \mathbb{C} \mathbb{P}^{n-k}$ be oriented, monotone Lagrangian submanifolds. Denote by pr: $\left(\mu_{k+1} \times \cdots \times \mu_{n}\right)^{-1}\left(\frac{\pi}{n+1}, \ldots, \frac{\pi}{n+1}\right) \rightarrow$ $\mathbb{C} \mathbb{P}^{k}$ and $\mathrm{pr}^{\prime}:\left(\mu_{1} \times \cdots \times \mu_{k}\right)^{-1}\left(\frac{\pi}{n+1}, \ldots, \frac{\pi}{n+1}\right) \rightarrow \mathbb{C P}^{n-k}$ the reductions of $\mathbb{C P}^{n}$ at complementary monotone level sets. Then $\operatorname{pr}^{-1}(L) \subset \mathbb{C} \mathbb{P}^{n}$ and $\operatorname{pr}^{\prime-1}\left(L^{\prime}\right) \subset \mathbb{C} \mathbb{P}^{n}$ are monotone Lagrangian submanifolds and there exists a canonical chain homotopy equivalence

$$
C F\left(\operatorname{pr}^{-1}(L), \operatorname{pr}^{\prime-1}\left(L^{\prime}\right)\right) \cong C F\left(L, T_{\mathrm{Cl}}^{k}\right) \otimes C F\left(T_{\mathrm{Cl}}^{n-k}, L^{\prime}\right) .
$$

Here we assume that $\partial^{2}=0$ on either the left hand side or the ride hand side complex; otherwise this is an equivalence in the category of derived matrix factorizations.

Proof Denote by $\Sigma_{(1, \ldots, k)} \subset\left(\mathbb{C} \mathbb{P}^{n-k}\right)^{-} \times \mathbb{C P}^{n}$ the Lagrangian correspondence arising from reduction at the level set $\left(\mu_{1} \times \cdots \times \mu_{k}\right)^{-1}\left(\frac{\pi}{n+1}, \ldots, \frac{\pi}{n+1}\right) \subset \mathbb{C P}^{n}$. Then

$$
\begin{aligned}
\Sigma_{(k+1, \ldots, n)} \times \mathbb{C P}^{n} \Sigma_{(1, \ldots, k)}^{t} & \cong\left(\mu_{k+1} \times \cdots \mu_{n}\right)^{-1}\left(\frac{\pi}{n+1}, \ldots\right) \cap\left(\mu_{1} \times \cdots \mu_{k}\right)^{-1}\left(\frac{\pi}{n+1}, \ldots\right) \\
& =T_{\mathrm{Cl}}^{n}
\end{aligned}
$$

is transverse and embeds to $\Sigma_{(k+1, \ldots, n)} \circ \Sigma_{(1, \ldots, k)}^{t}=T_{\mathrm{Cl}}^{k} \times T_{\mathrm{Cl}}^{n-k} \subset \mathbb{C P}^{k} \times \mathbb{C P}^{n-k}$. Now in complete analogy to the proof of Theorem 6.2.1 above, we have a sequence of chain homotopy equivalences

$$
\begin{aligned}
C F\left(\operatorname{pr}^{-1}(L), \operatorname{pr}^{\prime-1}\left(L^{\prime}\right)\right) & =C F\left(L \circ \Sigma_{(k+1, \ldots, n)}, \Sigma_{(1, \ldots, k)}^{t} \circ L^{\prime}\right) \\
& \cong C F\left(L, \Sigma_{(k+1, \ldots, n)}, \Sigma_{(1, \ldots, k)}^{t}, L\right) \\
& \cong C F\left(L, \Sigma_{(k+1, \ldots, n)} \circ \Sigma_{(1, \ldots, k)}^{t}, L^{\prime}\right) \\
& \cong C F\left(L, T_{\mathrm{Cl}}^{k} \times T_{\mathrm{Cl}}^{n-k}, L^{\prime}\right) \\
& \cong C F\left(L, T_{\mathrm{Cl}}^{k}\right) \otimes C F\left(T_{\mathrm{Cl}}^{n-k}, L^{\prime}\right) .
\end{aligned}
$$

A special case of Theorem 6.2.2 arises from taking $L^{\prime}$ to be a Clifford torus, then

$$
H F\left(\operatorname{pr}^{-1}(L), T_{\mathrm{Cl}}^{n}\right) \cong H F\left(L, T_{\mathrm{Cl}}^{k}\right) \otimes H_{*}\left(T^{n-k}\right),
$$

where we used $H F\left(T_{\mathrm{Cl}}^{n-k}, T_{\mathrm{Cl}}^{n-k}\right) \cong H_{*}\left(T^{n-k}\right)$ by [4] or Theorem 6.2.1. Since the latter is torsion-free, the Künneth Theorem 5.2.6 indeed implies the above isomorphism of homologies. This applies, for example, to $L=\mathbb{R} \mathbb{P}^{1} \subset \mathbb{C P}^{1}$ and yields another Lagrangian torus $\operatorname{pr}^{-1}\left(\mathbb{R P}^{1}\right) \subset \mathbb{C P}^{n}$. Although it is Hamiltonian isotopic to the 
Clifford torus, we need not check this but can calculate directly the relative Floer cohomology

$$
H F\left(\operatorname{pr}^{-1}\left(\mathbb{R P}^{1}\right), T_{\mathrm{Cl}}^{n}\right) \cong H F\left(\mathbb{R} \mathbb{P}^{1}, T_{\mathrm{Cl}}^{1}\right) \otimes H_{*}\left(T^{n-1}\right) \cong H_{*}\left(T^{n}\right)
$$

as well as (for $n=2$ )

$$
\begin{aligned}
H F\left(\operatorname{pr}^{-1}\left(\mathbb{R P}^{1}\right), \operatorname{pr}^{\prime-1}\left(\mathbb{R P}^{1}\right)\right) & \cong H F\left(\mathbb{R P}^{1}, T_{\mathrm{Cl}}^{1}\right) \otimes H F\left(T_{\mathrm{Cl}}^{1}, \mathbb{R} \mathbb{P}^{1}\right) \\
& \cong H_{*}\left(T^{1}\right) \otimes H_{*}\left(T^{1}\right) \cong H_{*}\left(T^{2}\right) .
\end{aligned}
$$

More generally, we can apply Theorem 6.2.2 to odd real projective spaces $L=\mathbb{R}^{k} \subset$ $\mathbb{C P}^{k}$ for $k=2 \ell-1 \geq 3$ with $\mathbb{Z}_{2}$-coefficients. ${ }^{11}$ By explicit calculation due to Alston [2] the underlying Floer cohomology is

$$
H F\left(\mathbb{R P}^{2 \ell-1}, T_{\mathrm{Cl}}^{2 \ell-1} ; \mathbb{Z}_{2}\right) \cong \mathbb{Z}_{2}^{2^{\ell}} .
$$

Now our calculations in Theorem 6.2.2 provide with $\mathbb{Z}_{2}$-coefficients

$$
\begin{aligned}
H F\left(\mathrm{pr}^{-1}\left(\mathbb{R P}^{2 \ell-1}\right), T_{\mathrm{Cl}}^{n} ; \mathbb{Z}_{2}\right) & \cong H F\left(\mathbb{R P}^{2 \ell-1}, T_{\mathrm{Cl}}^{2 \ell-1} ; \mathbb{Z}_{2}\right) \otimes H_{*}\left(T_{\mathrm{Cl}}^{n-2 \ell+1} ; \mathbb{Z}_{2}\right) \\
& \cong \mathbb{Z}_{2}^{2^{\ell}+2(n-2 \ell+1)}
\end{aligned}
$$

as well as for even $n=2 m$

$$
\begin{aligned}
& H F\left(\operatorname{pr}^{-1}\left(\mathbb{R P}^{2 \ell-1}\right), \operatorname{pr}^{\prime-1}\left(\mathbb{R} \mathbb{P}^{2(m-\ell)+1}\right) ; \mathbb{Z}_{2}\right) \\
& \quad \cong H F\left(\mathbb{R} \mathbb{P}^{2 \ell-1}, T_{\mathrm{Cl}}^{2 \ell-1} ; \mathbb{Z}_{2}\right) \otimes H F\left(T_{\mathrm{Cl}}^{2(m-\ell)+1}, \mathbb{R P}^{2(m-\ell)+1} ; \mathbb{Z}_{2}\right) \cong \mathbb{Z}_{2}^{2^{\ell}+2^{m-\ell+1}}
\end{aligned}
$$

\subsection{Detecting nontrivial Floer cohomology of a Lagrangian correspon- dence}

In this section we provide a tool for deducing nontriviality of Floer cohomology and hence nondisplaceability of a Lagrangian correspondence itself (as Lagrangian submanifold).

Theorem 6.3.1 Let $L_{01} \subset M_{0}^{-} \times M_{1}$ be a Lagrangian correspondence. Suppose that there exists a Lagrangian submanifold $L_{1} \subset M_{1}$ such that $L_{0}:=L_{01} \circ L_{1}$ is an embedded composition and $H F\left(L_{0}, L_{0}\right) \neq 0$. Assume that $M_{0}, M_{1}$ satisfy (M1)(M2), $L_{0}, L_{1}, L_{01}$ satisfy (L1)-(L2), and $\left(L_{0} \times L_{1}, L_{01}\right)$ is a monotone pair in the sense of Definition 4.1.2 (b). Then the Lagrangian $L_{01} \subset M_{0}^{-} \times M_{1}$ has nonzero Floer cohomology $H F\left(L_{01}, L_{01}\right) \neq 0$.

11 The number of holomorphic discs through a generic point is 0 for $\mathbb{R P}^{k}$ (which has minimal Maslov number $k+1 \geq 3$ for $k \geq 2$ ) and it is $k+1$ for $T_{\mathrm{Cl}}^{k}$ by [4], hence $\partial^{2}=0$ on $C F\left(\mathbb{R P} \mathbb{P}^{k}, T_{\mathrm{Cl}}^{k}\right)$ only holds for odd $k$ and with $\mathbb{Z}_{2}$ coefficients. 
Proof The Floer homologies $H F\left(L_{0}, L_{0}\right) \cong H F\left(L_{0}, L_{01}, L_{1}\right)=H F\left(L_{0} \times L_{1}, L_{01}\right)$ are all well-defined, isomorphic, and nonzero by assumption. (The differential on all three squares to zero since the total disk count in the sense of (24) is $w\left(L_{0}\right)-w\left(L_{0}\right)=$ 0.) Now $H F\left(L_{0} \times L_{1}, L_{01}\right)$ is a module over $H F\left(L_{01}, L_{01}\right)$, where the multiplication is defined by counting pseudoholomorphic 3-gons; see eg Seidel [33] or our paper [39]. The unit $1_{L_{01}} \in H F\left(L_{01}, L_{01}\right)$ is defined by counting pseudoholomorphic 1-gons; it is nontrivial since it acts as identity on a nontrivial group. Hence $\operatorname{HF}\left(L_{01}, L_{01}\right)$ contains a nonzero element, as claimed.

Corollary 6.3.2 Let $\Sigma \subset M$ be the level set of the moment map of a Hamiltonian $G$ action. Suppose that $\Sigma$ contains a $G$-invariant Lagrangian submanifold $L \subset M$ such that $H F(L, L) \neq 0$ and $\operatorname{pr}(L) \subset \Sigma / G=M / / G$ is smooth. Assume that $M, M / / G$ satisfy (M1)-(M2), $\Sigma, L, \operatorname{pr}(L)$ satisfy $(\mathrm{L} 1)-(\mathrm{L} 2)$, and $(\operatorname{pr}(L) \times L, \Sigma)$ is a monotone pair in the sense of Definition 4.1.2 (b). Then $\operatorname{HF}(\Sigma, \Sigma) \neq 0$.

Proof This is a case of Theorem 6.3.1, where $L_{1}=\operatorname{pr}(L)$, and the composition $\Sigma \circ \operatorname{pr}(L)=\operatorname{pr}^{-1}(\operatorname{pr}(L))=L$ is automatically embedded.

The following example in case $k=n=2$ was initially pointed out to us in 2006 by Paul Seidel; we since learned of alternative proof methods by Biran-Cornea and Fukaya-OhOno-Ohta. We use the notation of Section 6.2; in particular $\Sigma_{(n)} \subset\left(\mathbb{C} \mathbb{P}^{n-1}\right)^{-} \times \mathbb{C} \mathbb{P}^{n}$ is a Lagrangian $2 n-1$-sphere arising from reduction at the level set $\mu_{n}^{-1}\left(\frac{\pi}{n+1}\right)$.

Corollary 6.3.3 For every $2 \leq k \leq n$, the Lagrangian embedding

$$
\Sigma_{(k, \ldots, n)} \subset\left(\mathbb{C} \mathbb{P}^{k-1}\right)^{-} \times \mathbb{C} \mathbb{P}^{n}
$$

of $\left(S^{1}\right)^{n-k} \times S^{2 k-1}$ is Hamiltonian nondisplaceable.

Proof By construction $\Sigma_{(k, \ldots, n)}$ is the correspondence arising from the level set of $\mu_{k} \times \cdots \times \mu_{n}$ at the level $\left(\frac{\pi}{n+1}, \ldots, \frac{\pi}{n+1}\right)$ which contains the nondisplaceable Clifford torus $T_{\mathrm{Cl}}^{n} \subset \mathbb{C P}^{n}$. The projection $\operatorname{pr}\left(T_{\mathrm{Cl}}^{n}\right)=T_{\mathrm{Cl}}^{n} \circ \Sigma_{(k, \ldots, n)}$ is the Clifford torus $T_{\mathrm{Cl}}^{k-1} \subset \mathbb{C P}^{k-1}$. The Clifford tori as well as $\Sigma_{(k, \ldots, n)}$ are monotone with minimal Maslov number 2 (but this is immaterial here since $\partial^{2}=0$ on the relevant Floer complex), and the monotonicity of the pair $\left(T_{\mathrm{Cl}}^{k-1} \times T_{\mathrm{Cl}}^{n}, \Sigma_{(k, \ldots, n)}\right)$ follows directly from the monotonicity of the factors and the torsion fundamental groups of complex projective space. Now Corollary 6.3.2 says that the nonvanishing of $H F\left(T_{\mathrm{Cl}}^{n}, T_{\mathrm{Cl}}^{n}\right) \neq 0$ (by [4] or Section 6.2) directly implies nonvanishing Floer cohomology $\operatorname{HF}\left(\Sigma_{(k, \ldots, n)}, \Sigma_{(k, \ldots, n)}\right) \neq 0$, and hence nondisplaceability.

To spell things out in this example, Theorem 5.4.1 provides an isomorphism

$$
H F\left(T_{\mathrm{Cl}}^{k-1} \times T_{\mathrm{Cl}}^{n}, \Sigma_{(k, \ldots, n)}\right)=H F\left(T_{\mathrm{Cl}}^{k-1}, \Sigma_{(k, \ldots, n)}, T_{\mathrm{Cl}}^{n}\right) \cong H F\left(T_{\mathrm{Cl}}^{n}, T_{\mathrm{Cl}}^{n}\right) \neq 0 .
$$




\subsection{Gysin sequence for spherically fibered Lagrangian correspondence}

In this section, we give a conjectural relation between Floer cohomology $H F\left(L, L^{\prime}\right)$ for $L, L^{\prime} \subset M_{0}$ and the Floer cohomology $H F\left(L_{01} \circ L, L_{01} \circ L^{\prime}\right)$ for the images in $M_{1}$ under a correspondence $L_{01} \subset M_{0}^{-} \times M_{1}$. Results of this type can be viewed as transfer of nondisplaceability results, in the sense that nontriviality of $H F\left(L, L^{\prime}\right)$ implies nontriviality of $H F\left(L_{01} \circ L, L_{01} \circ L^{\prime}\right)$ and hence nondisplaceability of $L_{01} \circ L$ from $L_{01} \circ L^{\prime}$ by Hamiltonian perturbation.

In our example, the Lagrangian correspondence arises from a spherically fibered coisotropic $\iota: C \rightarrow M$ with projection $\pi: C \rightarrow B$. The image of $C$ under $\iota \times \pi$ is a Lagrangian correspondence from $M$ to $B$, also denoted $C$. Our standing assumptions are compactness, orientability, and monotonicity, ie $M, B$, and $C$ satisfy (M1)-(M2) and (L1)-(L2) with a fixed $\tau \geq 0$. Perutz [24] proved the following analogue of the Gysin sequence.

Theorem 6.4.1 Suppose that the minimal Maslov number of $C$ is larger or equal to $\operatorname{codim}(C \subset M)+2$. Then there exists a long exact sequence

$$
\cdots \rightarrow H F(C, C) \rightarrow H F(\mathrm{Id}) \rightarrow H F(\mathrm{Id}) \rightarrow H F(C, C) \rightarrow \cdots
$$

where the map $H F(\mathrm{Id}) \rightarrow H F(\mathrm{Id})$ is quantum multiplication by the Euler class of $\pi$.

One naturally conjectures the following relative version (for example, compare the Seidel triangle with the relative version in [32]).

Conjecture 6.4.2 Let $L_{0}, L_{1} \subset B$ be a monotone pair of Lagrangian submanifolds satisfying (L1)-(L3). (Or replace (L3) by $w\left(L_{0}\right)=w\left(L_{1}\right)$.) Suppose that the minimal Maslov number of $C$ is at least $\operatorname{codim}(C \subset M)+2$ Then there exists a long exact sequence

$\cdots \rightarrow H F\left(L_{0}, C^{t}, C, L_{1}\right) \rightarrow H F\left(L_{0}, L_{1}\right) \rightarrow H F\left(L_{0}, L_{1}\right) \rightarrow H F\left(L_{0}, C^{t}, C, L_{1}\right) \rightarrow \cdots$

where the middle map is Floer theoretic multiplication ${ }^{12}$ by the Euler class of $\pi$.

The compositions $C \circ L_{1}$ and $L_{0} \circ C^{t}=\left(C \circ L_{0}\right)^{t}$ are clearly embedded. Hence Conjecture 6.4.2 together with Theorem 5.4.1 implies the following.

Corollary 6.4.3 Under the same assumptions as in Conjecture 6.4.2 there exists a long exact sequence

$\cdots \rightarrow H F\left(C \circ L_{0}, C \circ L_{1}\right) \rightarrow H F\left(L_{0}, L_{1}\right) \rightarrow H F\left(L_{0}, L_{1}\right) \rightarrow H F\left(C \circ L_{0}, C \circ L_{1}\right) \rightarrow \cdots$

12 This product is defined by counting pseudoholomorphic strips with boundary on $\left(L_{0}, L_{1}\right)$ and an internal puncture with asymptotics fixed by a given class in $H F\left(\Delta_{B}\right) \cong H(B)$. 
In particular, we obtain a "transfer of nondisplaceability" result if the Euler class vanishes.

Corollary 6.4.4 With the same assumptions as in Conjecture 6.4.2, if the Euler class of $\pi: C \rightarrow B$ is zero, then $H F\left(C \circ L_{0}, C \circ L_{1}\right)$ is isomorphic to two copies of $H F\left(L_{0}, L_{1}\right)$.

Example 6.4.5 Suppose that $M$ is a monotone Hamiltonian $G=\mathrm{SU}(2)$ manifold, with moment map $\Phi$, and $\Phi^{-1}(0)$ is an SU(2)-bundle over the symplectic quotient $M / / G$.

Let $\left(L_{0}, L_{1}\right)$ be a monotone pair of $G$-invariant Lagrangians contained in the zero level set and with minimal Maslov number at least three. Necessarily each $L_{j}$ is a principal $\mathrm{SU}(2)$ bundle over $L_{j} / G \subset M / / G$. Suppose that the minimal Maslov number of $\Phi^{-1}(0)$, considered as a Lagrangian in $M^{-} \times M / / G$, is at least 5 . Then there is a long exact sequence

$\cdots \rightarrow H F\left(L_{0}, L_{1}\right) \rightarrow H F\left(L_{0} / G, L_{1} / G\right) \rightarrow H F\left(L_{0} / G, L_{1} / G\right) \rightarrow H F\left(L_{0}, L_{1}\right) \rightarrow \cdots$.

In particular, if $M \rightarrow M / / G$ is a trivial $G$-bundle, then $\operatorname{HF}\left(L_{0}, L_{1}\right)$ is isomorphic to two copies of $H F\left(L_{0} / G, L_{1} / G\right)$.

\section{References}

[1] M Abouzaid, I Smith, Homological symmetry for the four-torus arXiv:0903.3065

[2] G Alston, Lagrangian Floer homology of the Clifford torus and real projective space in odd dimensions arXiv:0902.0197

[3] P Biran, M Entov, L Polterovich, Calabi quasimorphisms for the symplectic ball, Commun. Contemp. Math. 6 (2004) 793-802 MR2100764

[4] C-H Cho, Holomorphic discs, spin structures, and Floer cohomology of the Clifford torus, Int. Math. Res. Not. (2004) 1803-1843 MR2057871

[5] S K Donaldson, Floer homology groups in Yang-Mills theory, Cambridge Tracts in Math. 147, Cambridge Univ. Press (2002) MR1883043 With the assistance of M Furuta and D Kotschick

[6] A Floer, Morse theory for Lagrangian intersections, J. Differential Geom. 28 (1988) 513-547 MR965228

[7] A Floer, A relative Morse index for the symplectic action, Comm. Pure Appl. Math. 41 (1988) 393-407 MR933228

[8] A Floer, The unregularized gradient flow of the symplectic action, Comm. Pure Appl. Math. 41 (1988) 775-813 MR948771 
[9] A Floer, H Hofer, D Salamon, Transversality in elliptic Morse theory for the symplectic action, Duke Math. J. 80 (1995) 251-292 MR1360618

[10] K Fukaya, Y-G Oh, H Ohta, K Ono, Lagrangian intersection Floer theoryanomaly and obstruction, Preprint Available at http://www.kusm.kyoto-u.ac.jp/ fukaya/fooo.dvi

[11] A R P Gaio, D A Salamon, Gromov-Witten invariants of symplectic quotients and adiabatic limits, J. Symplectic Geom. 3 (2005) 55-159 MR2198773

[12] V Guillemin, S Sternberg, The moment map revisited, J. Differential Geom. 69 (2005) 137-162 MR2169585

[13] F C Kirwan, Cohomology of quotients in symplectic and algebraic geometry, Math. Notes 31, Princeton Univ. Press (1984) MR766741

[14] P B Kronheimer, T S Mrowka, Knot homology groups from instantons arXiv: 0806.1053

[15] D Kwon, Y-G Oh, Structure of the image of (pseudo)-holomorphic discs with totally real boundary condition, Comm. Anal. Geom. 8 (2000) 31-82 MR1730896 Appendix 1 by J-P Rosay

[16] Y Lekili, Heegaard Floer homology of broken fibrations over the circle arXiv: 0903.1773

[17] R B Lockhart, R C McOwen, Elliptic differential operators on noncompact manifolds, Ann. Scuola Norm. Sup. Pisa Cl. Sci. (4) 12 (1985) 409-447 MR837256

[18] D McDuff, D Salamon, J-holomorphic curves and symplectic topology, Amer. Math. Soc. Coll. Publ. 52, Amer. Math. Soc. (2004) MR2045629

[19] E Meinrenken, C Woodward, Canonical bundles for Hamiltonian loop group manifolds, Pacific J. Math. 198 (2001) 477-487 MR1835519

[20] Y-G Oh, Floer homology and symplectic topology, in preparation

[21] Y-G Oh, Removal of boundary singularities of pseudo-holomorphic curves with Lagrangian boundary conditions, Comm. Pure Appl. Math. 45 (1992) 121-139 MR1135926

[22] Y-G Oh, Floer cohomology of Lagrangian intersections and pseudo-holomorphic disks. I, Comm. Pure Appl. Math. 46 (1993) 949-993 MR1223659

[23] Y-G Oh, On the structure of pseudo-holomorphic discs with totally real boundary conditions, J. Geom. Anal. 7 (1997) 305-327 MR1646780

[24] T Perutz, A symplectic Gysin sequence arXiv:0807.1863

[25] T Perutz, Lagrangian matching invariants for fibred four-manifolds. I, Geom. Topol. 11 (2007) 759-828 MR2302502

[26] R Rezazadegan, Seidel-Smith cohomology for tangles, Selecta Math. (N.S.) 15 (2009) 487-518 MR2551191 
[27] J Robbin, D Salamon, The Maslov index for paths, Topology 32 (1993) 827-844 MR1241874

[28] H L Royden, Real analysis, third edition, Macmillan, New York (1988) MR1013117

[29] D Salamon, Lectures on Floer homology, from: "Symplectic geometry and topology (Park City, UT, 1997)", (Y Eliashberg, L Traynor, editors), IAS/Park City Math. Ser. 7, Amer. Math. Soc. (1999) 143-229 MR1702944

[30] M Schwarz, Cohomology operations from $S^{1}$-cobordisms in Floer homology, $\mathrm{PhD}$ thesis, ETH Zürich (1995) Available at http://www.math.uni-leipzig.de/ schwarz/

[31] P Seidel, Graded Lagrangian submanifolds, Bull. Soc. Math. France 128 (2000) 103149 MR1765826

[32] P Seidel, A long exact sequence for symplectic Floer cohomology, Topology 42 (2003) 1003-1063 MR1978046

[33] P Seidel, Fukaya categories and Picard-Lefschetz theory, Zurich Lectures in Advanced Math., European Math. Soc., Zürich (2008) MR2441780

[34] P Seidel, Lectures on four-dimensional Dehn twists, from: "Symplectic 4-manifolds and algebraic surfaces", (F Catanese, G Tian, editors), Lecture Notes in Math. 1938, Springer, Berlin (2008) 231-267 MR2441414

[35] E H Spanier, Algebraic topology, Springer, New York (1981) MR666554

[36] C Viterbo, Intersection de sous-variétés lagrangiennes, fonctionnelles d'action et indice des systèmes hamiltoniens, Bull. Soc. Math. France 115 (1987) 361-390 MR926533

[37] K Wehrheim, Lagrangian boundary conditions for anti-self-dual instantons and the Atiyah-Floer conjecture, J. Symplectic Geom. 3 (2005) 703-747 MR2235859Conference on Symplectic Topology

[38] K Wehrheim, C T Woodward, Floer cohomology and geometric composition of Lagrangian correspondences arXiv:0905.1368

[39] K Wehrheim, C T Woodward, Functoriality for Lagrangian correspondences in Floer theory arXiv:0708.2851

[40] K Wehrheim, C T Woodward, Orientations for pseudoholomorphic quilts, in preparation

[41] K Wehrheim, C T Woodward, Pseudoholomorphic quilts arXiv:0905.1369

[42] K Wehrheim, C T Woodward, Floer field theory, Preprint (2008)

[43] K Wehrheim, C T Woodward, Floer field theory for tangles, Preprint (2008)

[44] A Weinstein, Lectures on symplectic manifolds, CBMS Regional Conference Ser. in Math. 29, Amer. Math. Soc. (1979) MR598470 
[45] A Weinstein, The symplectic "category", from: "Differential geometric methods in mathematical physics (Clausthal, 1980)", (P García, L A Pérez-Rendón, J M Souriau, editors), Lecture Notes in Math. 905, Springer, Berlin (1982) 45-51 MR657441

Department of Mathematics, Massachusetts Institute of Technology Cambridge, MA 02139, USA

Department of Mathematics, Rutgers University

Piscataway, NJ 08854, USA

katrin@math.mit.edu, ctw@math.rutgers.edu

www-math.mit.edu/ katrin, www.math.rutgers.edu/ ctw

Proposed: Leonid Polterovich

Received: 14 May 2009

Seconded: Danny Calegari, Peter Teichner

Revised: 4 January 2009 